San Jose State University

SJSU ScholarWorks

Master's Theses

Master's Theses and Graduate Research

Summer 2011

\title{
The synthesis and characterization of endcapped silica hydride stationary phase
}

Prema Gangadhara

San Jose State University

Follow this and additional works at: https://scholarworks.sjsu.edu/etd_theses

\section{Recommended Citation}

Gangadhara, Prema, "The synthesis and characterization of endcapped silica hydride stationary phase" (2011). Master's Theses. 4048.

DOI: https://doi.org/10.31979/etd.abmc-mma7

https://scholarworks.sjsu.edu/etd_theses/4048

This Thesis is brought to you for free and open access by the Master's Theses and Graduate Research at SJSU ScholarWorks. It has been accepted for inclusion in Master's Theses by an authorized administrator of SJSU ScholarWorks. For more information, please contact scholarworks@sjsu.edu. 


\title{
THE SYNTHESIS AND CHARACTERIZATION OF ENDCAPPED SILICA HYDRIDE STATIONARY PHASE
}

\author{
A Thesis \\ Presented to \\ The Faculty of the Department of Chemistry \\ San Jose State University \\ In Partial Fulfillment \\ of the Requirements for the Degree \\ Master of Science
}

by

Prema Gangadhara

August 2011 
(C) 2011

Prema Gangadhara

ALL RIGHTS RESERVED 
The Designated Thesis Committee Approves the Thesis Titled

\title{
THE SYNTHESIS AND CHARACTERIZATION OF ENDCAPPED SILICA HYDRIDE STATIONARY PHASE
}

\author{
by \\ Prema Gangadhara \\ APPROVED FOR THE DEPARTMENT OF CHEMISTRY \\ SAN JOSE STATE UNIVERSITY
}

August 2011
Dr. Joseph J. Pesek
Department of Chemistry
Dr. Roger Terrill
Department of Chemistry
Dr. Brooke Lustig
Department of Chemistry 


\title{
ABSTRACT
}

\section{THE SYNTHESIS AND CHARACTERIZATION OF ENDCAPPED SILICA HYDRIDE STATIONARY PHASE}

\author{
by Prema Gangadhara
}

The silanization/hydrosilation process was developed to overcome some of the drawbacks associated with the use of organosilane reagents for the synthesis of high pressure liquid chromatography (HPLC) stationary phases. The first step is called silanization, in which silica or other oxides react with triethoxysilane (TES), resulting in the formation of a silica hydride surface. Endcapping the stationary phase using trimethylchlorosilane (TMCS) and hexamethyldisilazane (HMDS) eliminates the unreacted and accessible silanols on the silica hydride intermediate.

This research employed the modification of bare silica to silica hydride via silanization. Endcapping after silanization eliminated accessible and unreacted silanols. Endcapping was confirmed by using diffuse reflectance infrared Fourier transform (DRIFT) spectroscopy. The stationary phase was characterized for its hydrophobicity and silanophilicity properties. The effectiveness of the stationary phase was determined for its aqueous normal phase and reversed-phase chromatographic behavior. The performance was compared with the performance of C8 silica hydride column under similar conditions. This research confirmed that endcapped columns could be used in the aqueous normal phase and reversed-phase modes. Endcapping increased the hydrophobicity of the surface, which in turn increased the efficiency of separation for nonpolar solutes. 


\section{ACKNOWLEDGEMENTS}

I would particularly like to thank Dr. Pesek, my research advisor, for giving me the opportunity to work on my favorite subject, for pointing me in the right direction, and in helping with ideas and suggestions. I would also like to thank Dr. Maria T. Matyska for all the suggestions and guidelines. Then I would like to thank my committee members Dr. Roger Terrill and Dr. Brooke Lustig for providing feedback on my work. I also would like to thank Dr. Palani Balu who helped me with my research and to gain knowledge of different chromatographic techniques. I thank my co-workers Anthony Tomlinson and Priya Natarajan for the helpful discussions and arguments which led me to think with varying perspectives and to come up with good solutions.

I greatly appreciate the support of my friends and family, and especially would like to thank my parents and brothers for their advice, support, and constant encouragement. 


\section{TABLE OF CONTENTS}

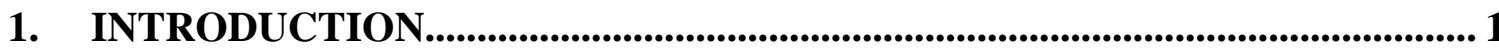

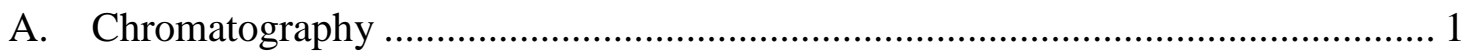

B. Modes of Separation ............................................................................... 2

1. Normal Phase High Performance Liquid Chromatography............................... 2

2. Reversed-Phase High Performance Liquid Chromatography ........................... 2

3. Hydrophilic Interaction Liquid Chromatography ....................................... 2

4. Aqueous Normal Phase Chromatography................................................... 3

C. Resolution and Column Efficiency ............................................................ 4

D. Column Material .................................................................................... 5

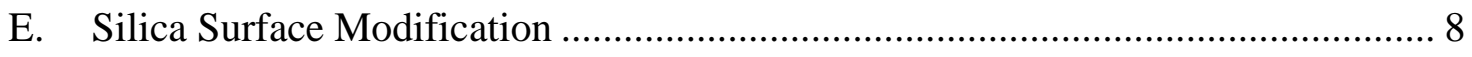

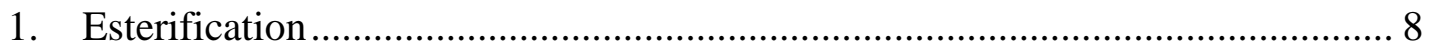

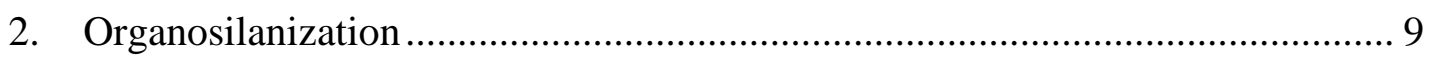

3. Chlorination Reaction Followed by a Reaction with Grignard Reagents or an Organolithium Compounds ............................................................... 9

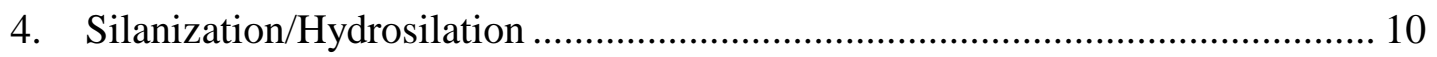

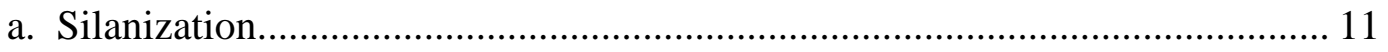

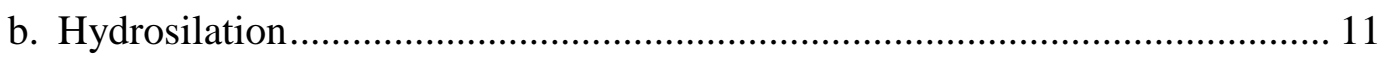

F. Endcapped Silica Hydride Stationary Phase ................................................. 14

G. Advantages of Using Hydride-based Stationary Phases ................................. 15 


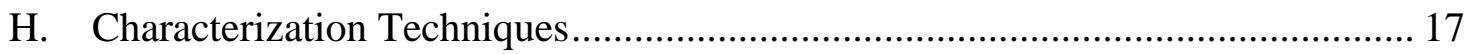

1. Diffuse Reflectance Fourier Transform Infrared Spectroscopy ........................ 17

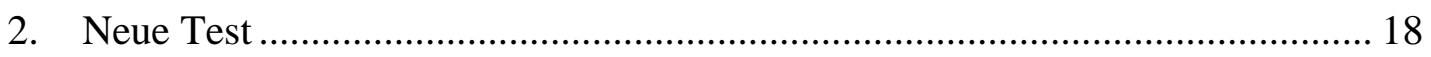

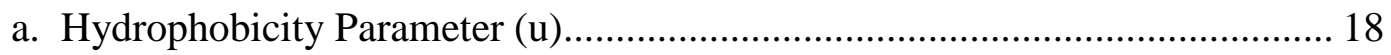

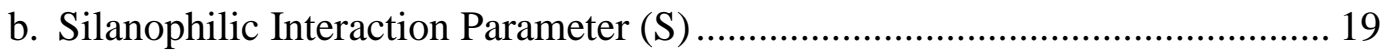

3. Elemental Analysis ................................................................................. 19

4. High Performance Liquid Chromatography .................................................... 19

a. Evaporative Light Scattering Detector ........................................................... 22

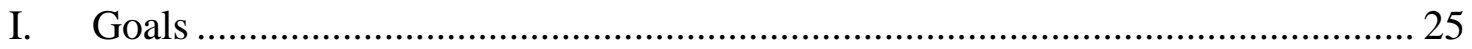

II. EXPERIMENTAL ............................................................................................................. 27

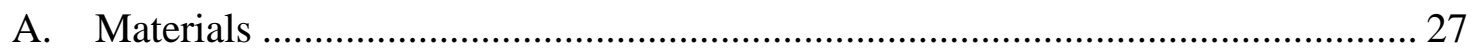

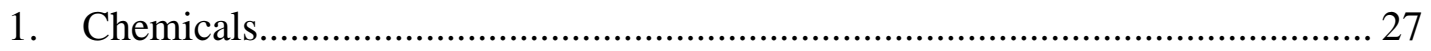

2. Compounds Used for Chromatographic Characterization................................... 29

3. Structures of the Compounds Used for Neue Test ............................................... 31

4. Structures of Compounds Used for Chromatographic Characterization ............ 32

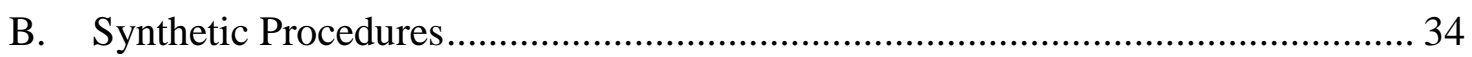

1. Synthesis of Silica Hydride via Silanization......................................................... 34

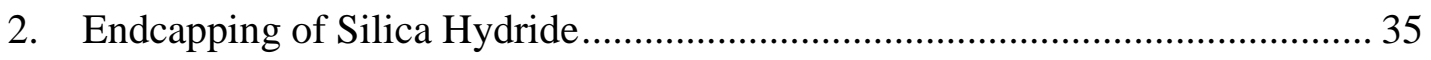

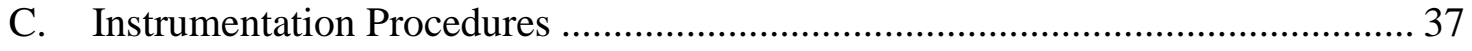

1. Diffuse Reflectance Infrared Fourier Transform Spectroscopy .......................... 37

2. Column Packing .............................................................................................. 37 
3. High Performance Liquid Chromatography

III. RESULTS AND DISCUSSIONS .................................................................... 39

A. Spectroscopic Characterization of Bonded Stationary Phases........................... 39

1. Diffuse Reflectance Infrared Fourier Transform Spectra .............................. 39

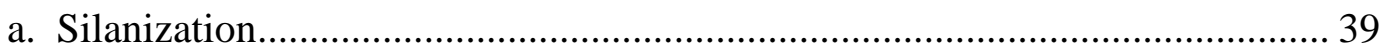

b. Endcapped Silica Hydride................................................................. 40

B. Chromatographic Characterization of Bonded Stationary Phase....................... 41

1. Characterization of HPLC Packing by Neue Test .......................................... 42

a. Hydrophobicity Result of Endcapped Si-H Stationary Phase ........................ 44

b. Silanophilicity Result of Endcapped Si-H Stationary Phase ......................... 45

c. Hydrophobicity Result of C8 Stationary Phase ......................................... 46

d. Silanophilicity Result of C8 Stationary Phase ............................................ 48

2. ANP and RP Behavior of Endcapped Si-H Using an ELSD Detector ..............50

a. Aqueous Normal Phase Behavior of Citric Acid ...................................... 50

b. Reversed-Phase Behavior of Citric Acid .................................................. 56

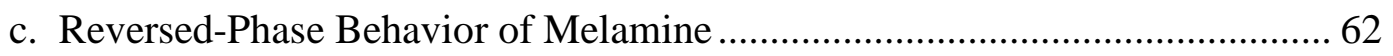

d. Aqueous Normal Phase Behavior of Melamine .......................................... 67

e. Aqueous Normal Phase Behavior of Phenylalanine .................................. 70

3. ANP and RP Behavior of Endcapped Si-H Using UV Detection.................... 72

a. Aqueous Normal Phase Behavior of Phenylalanine .................................. 75

b. Aqueous Normal Phase Behavior of Niacinic Acid................................... 78

c. Aqueous Normal Phase Behavior of Lisinopril ...................................... 82 
d. ANP Separation of Two Compounds Using an Isocratic Method

e. RP Behavior of Endcapped Si-H Using UV Detection Under Isocratic and

Gradient Conditions .......................................................................... 87

4. ANP and RP Behavior of Si-H based C8 Column Using UV Detection........... 94

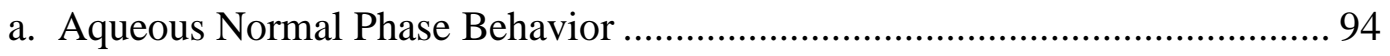

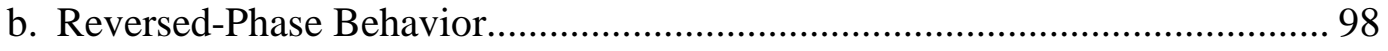

5. Comparison of Endcapped Si-H Column and C8 Column Separation

Under RP Conditions ....................................................................... 106

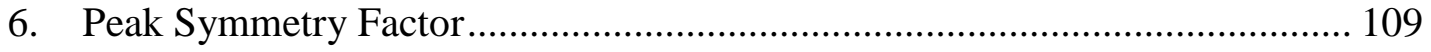

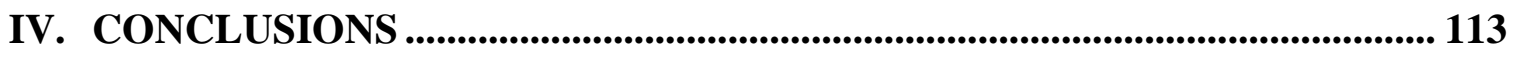

REFERENCES.............................................................................................................. 114 


\section{LIST OF FIGURES}

Figure 1. Active groups on a silica surface .............................................................. 7

Figure 2. Retention map for RP and ANP region on a Si-H based stationary

phase. 13

Figure 3. The structure of normal silica surface and Si-H surface .............................. 16

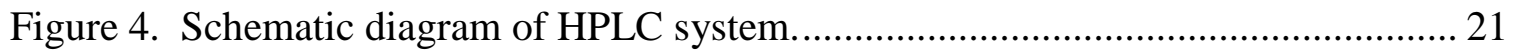

Figure 5. Schematic diagram of nebulization spray chamber .................................. 25

Figure 6. Structures of Neue test compounds ...................................................... 31

Figure 7. Structures of compounds used for chromatographic characterization ............. 34

Figure 8. DRIFT spectrum of silica hydride stationary phase .................................. 40

Figure 9. DRIFT spectrum of endcapped Si-H stationary phase ............................... 41

Figure 10. Hydrophobicity test on endcapped silica hydride phase ............................. 44

Figure 11. Silanophobicity test on endcapped silica hydride phase ............................ 45

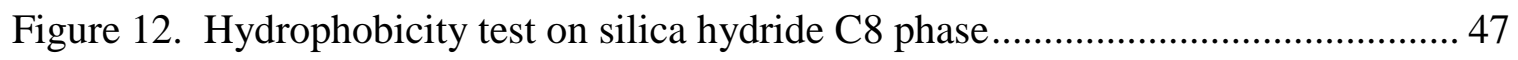

Figure 13. Silanophobicity test on silica hydride C8 column.................................... 48

Figure 14. Chromatogram of citric acid on endcapped Si-H stationary phase.

Mobile phase: acetonitrile $+0.1 \%$ formic acid (FA) .............................. 51

Figure 15. Chromatogram of citric acid on endcapped Si-H stationary phase.

Mobile phase: $10: 90$ water $+0.1 \%$ formic acid / acetonitrile $+0.1 \%$

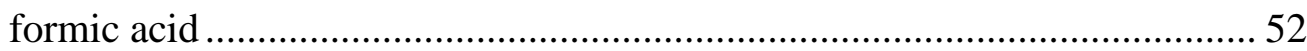


Figure 16. Chromatogram of citric acid on endcapped Si-H stationary phase.

Mobile phase: $20: 80$ water $+0.1 \%$ formic acid / acetonitrile $+0.1 \%$

formic acid

Figure 17. Chromatogram of citric acid on endcapped Si-H stationary phase.

Mobile phase: $30: 70$ water $+0.1 \%$ formic acid / acetonitrile $+0.1 \%$

formic acid

Figure 18. Chromatogram of citric acid on endcapped Si-H stationary phase.

Mobile phase: $40: 60$ water $+0.1 \%$ formic acid / acetonitrile $+0.1 \%$

formic acid 55

Figure 19. Chromatogram of citric acid on endcapped Si-H stationary phase.

Mobile phase: $60: 40$ water $+0.1 \%$ formic acid / acetonitrile $+0.1 \%$

formic acid 56

Figure 20. Chromatogram of citric acid on endcapped Si-H stationary phase.

Mobile phase: $70: 30$ water $+0.1 \%$ formic acid / acetonitrile $+0.1 \%$

formic acid

Figure 21. Chromatogram of citric acid on endcapped Si-H stationary phase.

Mobile phase: $80: 20$ water $+0.1 \%$ formic acid / acetonitrile $+0.1 \%$

formic acid 58

Figure 22. Chromatogram of citric acid on endcapped Si-H stationary phase.

Mobile phase: $90: 10$ water $+0.1 \%$ formic acid / acetonitrile $+0.1 \%$

formic acid 
Figure 23. Chromatogram of citric acid on endcapped Si-H stationary phase.

Mobile phase: acetonitrile $+0.1 \%$ formic acid 60

Figure 24. Retention map of citric acid for ANP and RP conditions 61

Figure 25. Chromatogram of melamine on endcapped Si-H stationary phase.

Mobile phase: $90: 10$ water $+0.1 \%$ formic acid / acetonitrile $+0.1 \%$

formic acid

Figure 26. Chromatogram of melamine on endcapped Si-H stationary phase.

Mobile phase: $80: 20$ water $+0.1 \%$ formic acid / acetonitrile $+0.1 \%$

formic acid

Figure 27. Chromatogram of melamine on endcapped Si-H stationary phase.

Mobile phase: $70: 30$ water $+0.1 \%$ formic acid / acetonitrile $+0.1 \%$

formic acid

Figure 28. Chromatogram of melamine on endcapped Si-H stationary phase.

Mobile phase: $60: 40$ water $+0.1 \%$ formic acid / acetonitrile $+0.1 \%$

formic acid

Figure 29. Chromatogram of melamine on endcapped Si-H stationary phase.

Mobile phase: $50: 50$ water $+0.1 \%$ formic acid $/$ acetonitrile $+0.1 \%$

formic acid 66

Figure 30. Chromatogram of melamine on endcapped Si-H stationary phase.

Mobile phase: $40: 60$ water $+0.1 \%$ formic acid / acetonitrile $+0.1 \%$

formic acid

67 
Figure 31. Chromatogram of melamine on endcapped Si-H stationary phase.

Mobile phase: $20: 80$ water $+0.1 \%$ formic acid / acetonitrile $+0.1 \%$

formic acid 68

Figure 32. Retention map of melamine for ANP and RP conditions

Figure 33. ANP retention of phenylalanine on endcapped Si-H stationary phase 70

Figure 34. Chromatogram of phenylalanine on endcapped Si-H SP. Mobile phase: $50: 50$ water $+0.1 \%$ formic acid / acetonitrile $+0.1 \%$ formic acid.

Figure 35. Chromatogram of phenylalanine on endcapped Si-H stationary phase. Mobile phase: $40: 60$ water $+0.1 \%$ formic acid / acetonitrile $+0.1 \%$ FA

Figure 36. Chromatogram of phenylalanine on endcapped silica hydride SP. Mobile phase: $30: 70$ water $+0.1 \%$ formic acid / acetonitrile $+0.1 \%$ formic acid 76

Figure 37. Chromatogram of phenylalanine on endcapped silica hydride SP. Mobile phase: $20: 80$ water $+0.1 \%$ formic acid / acetonitrile $+0.1 \%$ formic acid 77

Figure 38. Chromatogram of niacinic acid on endcapped silica hydride SP. Mobile phase: $40: 60$ water $+0.1 \%$ formic acid / acetonitrile $+0.1 \%$ formic acid 79 
Figure 39. Chromatogram of niacinic acid on endcapped silica hydride SP.

Mobile phase: $30: 70$ water $+0.1 \%$ formic acid / acetonitrile $+0.1 \%$

formic acid

Figure 40. ANP retention of niacinic acid on endcapped silica hydride SP.

Mobile phase: $20: 80$ water $+0.1 \%$ formic acid / acetonitrile $+0.1 \%$

formic acid 80

Figure 41. ANP retention of niacinic acid on endcapped silica hydride SP.

Mobile phase: $10: 90$ water $+0.1 \%$ formic acid / acetonitrile $+0.1 \%$

formic acid 80

Figure 42. ANP retention of niacinic acid on endcapped silica hydride SP.

Mobile phase: $5: 95$ ater $+0.1 \%$ formic acid / acetonitrile $+0.1 \%$

formic acid 81

Figure 43. ANP retention of lisinopril on endcapped Si-H stationary phase.

Mobile phase: $20: 80$ water $+0.1 \%$ trifluoroacetic acid (TFA) /

acetonitrile $+0.1 \%$ trifluoroacetic acid

Figure 44. Chromatogram of lisinopril on endcapped Si-H stationary phase.

Mobile phase: $10: 90$ water $+0.1 \%$ TFA / acetonitrile $+0.1 \%$ TFA

Figure 45. ANP retention of lisinopril on endcapped Si-H stationary phase.

Mobile phase: 5:95 water $+0.1 \%$ TFA / acetonitrile $+0.1 \%$ TFA

Figure 46. ANP separation of lipoic acid and lisinopril. Mobile phase: 95:5 acetonitrile/water $+0.1 \%$ TFA 85 
Figure 47. ANP separation of dimethylthiourea and raloxifene. Mobile phase: 95:5 acetonitrile/water $+0.1 \%$ TFA 86

Figure 48. RP retention of lisinopril on endcapped Si-H. Mobile phase: 75:25 water/acetonitrile $+0.1 \%$ TFA 87

Figure 49. RP retention of dimethylthiourea on endcapped $\mathrm{Si}-\mathrm{H}$ column using $100 \%$ water $+0.1 \%$ TFA 88

Figure 50. RP retetnion of raloxifene on endcapped $\mathrm{Si}-\mathrm{H}$ column 89

Figure 51. Chromatogram of raloxifene on the endcapped $\mathrm{Si}-\mathrm{H}$ column in RP mode 89

Figure 52. Chromatogram of lipoic acid on the endcapped Si-H column in RP mode 90

Figure 53. RP isocratic separation of lisinopril and lipoic acid. Mobile phase:

75:25 water/ acetonitrile $+0.1 \%$ TFA 91

Figure 54. RP gradient separation of dimethylthiourea and raloxifene 92

Figure 55. ANP isocratic retention of phenylalanine on the Si-H C8 column. The isocratic composition used is $60: 40$ acetonitrile/water $+0.1 \%$ TFA 94

Figure 56. ANP isocratic retention of dimethylthiourea on the Si-H based C8 column. The isocratic composition used is 90:10 acetonitrile/water + $0.1 \%$ TFA 95

Figure 57. ANP isocratic retention of raloxifene on the Si-H based C8 column. The isocratic composition used is $95: 5$ acetonitrile/water $+0.1 \%$ TFA 96 
Figure 58. ANP isocratic retention of raloxifene on the Si-H based C8 column.

The isocratic composition used is $80: 20$ acetonitrile/water $+0.1 \%$

TFA

Figure 59. ANP retention of lipoic acid on the Si-H C8 column. The isocratic composition used is $80: 20$ acetonitrile/water $+0.1 \%$ TFA

Figure 60. ANP retention of lipoic acid on the $\mathrm{Si}-\mathrm{H} \mathrm{C8}$ column. The isocratic composition used is $95: 5$ acetonitrile/water $+0.1 \%$ TFA 97

Figure 61. RP retention of niacinic acid on the $\mathrm{Si}-\mathrm{H} \mathrm{C} 8$ column. The isocratic composition used is $30: 70$ acetonitrile/water $+0.1 \%$ TFA 98

Figure 62. RP retention of lisinopril on the Si-H C8 column. Mobile Phase:

45:55 acetonitrile/water $+0.1 \%$ TFA 99

Figure 63. RP gradient retention of triphenylphosphine on the Si-H C8 column 100

Figure 64. RP gradient retention of triphenylphosphine on the Si-H C8 column 101

Figure 65. RP gradient retention of raloxifene on the Si-H C8 column 102

Figure 66. RP gradient retention of raloxifene on the Si-H C8 column 103

Figure 67. RP gradient separation of lisinopril and raloxifene on Si-H C8 column...... 104

Figure 68. RP gradient separation of lisinopril and lipoic acid on Si-H C8 column ..... 105

Figure 69. The overlay of RP chromatograms of raloxifene for the endcapped Si$\mathrm{H}$ column and the $\mathrm{C} 8$ column 107

Figure 70. The overlay of RP chromatograms of raloxifene for the endcapped SiH column and the $\mathrm{C} 8$ column . 108

Figure 71. Determination of peak symmetry factor 110 
Figure 72. Chromatographic determination of asymmetry factors of the compounds melamine, citric acid and phenylalanine on the endcapped Si-H column using isocratic conditions and the ELSD detector ......

Figure 73. Chromatographic determination of asymmetry factors of the compounds niacinic acid and phenylalanine on the endcapped $\mathrm{Si}-\mathrm{H}$ column using isocratic conditions and UV detection 112 


\section{LIST OF TABLES}

Table 1. Type of silica used for the silica hydride synthesis: .................................... 27

Table 2. Chemicals used for synthesis and characterization: ................................... 28

Table 3. Chemicals used for hydrophobicity and silanophilicity test .......................... 29

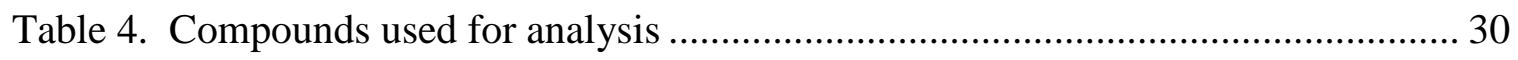

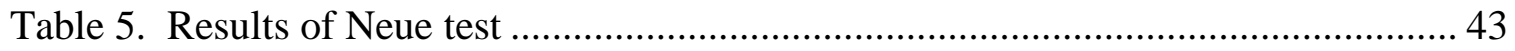

Table 6. Summary of hydrophobicity and silanophilicity results of C8 and

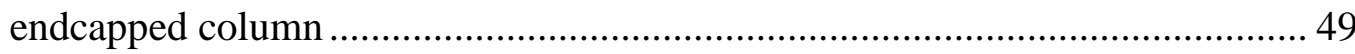

Table 7. Mobile phase gradient 1 used for the analysis ............................................. 73

Table 8. Mobile phase gradient 2 used for the analysis ........................................... 74

Table 9. Summary of isocratic elution data for phenylalanine …............................. 77

Table 10. Summary of isocratic elution data for niacinic acid ................................ 81

Table 11. Summary of isocratic elution data for lisinopril ....................................... 84

Table 12. Summary of isocratic elution data for Phenylalanine .................................. 95 


\section{INTRODUCTION}

\section{A. Chromatography}

Chromatography is a separation technique that has applications in all branches of science. The separation is based on a physical property in which the stationary and mobile phases are brought into contact and the sample mixture is separated based on its affinity towards a mobile phase and/or a stationary phase. The technique can be classified by the types of mobile and stationary phases used and the levels of equilibrium involved during the transfer of solutes between the two phases. Separation by chromatography can be classified into many different types on the basis of physical state of the mobile phase, the separation mechanism, and the shape of the chromatographic bed. A few of the separation techniques are adsorption chromatography, partition chromatography, size exclusion chromatography, affinity chromatography, and ionexchange chromatography. The applications of some of the separation techniques are as follows: gas chromatography is used mainly for volatile compounds; ion exchange chromatography is used mainly for separating ions; liquid chromatography is used for separating non-volatile compounds; size exclusion chromatography is used for separating compounds based on molecular weight [1-4]. 


\section{B. Modes of Separation}

\section{Normal Phase High Performance Liquid Chromatography}

In normal phase chromatography, the stationary phase is strongly polar and the mobile phase is nonpolar. The stationary phase usually consists of silica, cyano, diol, or amino groups; therefore polar compounds are retained longer than the nonpolar compounds. The retention of the compounds is governed by an adsorption mechanism on the column. The mobile phase usually consists of an organic solvent combination of a very nonpolar liquid with a slightly more polar solvent such as hexane/ethyl acetate or hexane/isopropanol. The main limitation of this separation is that it cannot be used with aqueous solvents [1-4].

\section{Reversed-Phase High Performance Liquid Chromatography}

In reversed-phase (RP) HPLC, the stationary phase is nonpolar in nature, whereas the mobile phase is polar. The stationary phase is usually made of nonpolar ligands bonded to a polar matrix; therefore nonpolar compounds that interact with the stationary phase are retained longer in the column and are better separated. In reversed phase, the separation is mainly governed by a partitioning process rather than by adsorption [1-4].

\section{Hydrophilic Interaction Liquid Chromatography}

Hydrophilic interaction liquid chromatography (HILIC) is normal phase chromatography that is used to separate polar compounds. HILIC provides the advantage 
of having aqueous and organic components in the mobile phase. This unique feature distinguishes it from normal phase chromatography. The chromatographic surface used for the stationary phase can be neutral, polar, or ionic. The main draw back with HILIC separation is that it can only separate polar compounds and cannot be used for nonpolar compound separation [1-4].

\section{Aqueous Normal Phase Chromatography}

A silica hydride ( $\mathrm{Si}-\mathrm{H})$ stationary phase is used in aqueous normal phase (ANP) as an alternative to other types of silica materials. The technique is used for separating both polar and nonpolar compounds. This unique stationary phase has many important advantages over ordinary silica columns in which most of the adsorptive properties are controlled by silanol groups. Si-H can separate both acidic and basic compounds because of its unique capability to use a wide range of mobile phase compositions, from $100 \%$ aqueous to pure nonpolar organic solvents.

Reversed-phase and aqueous normal phase separations are complementary to each

other as water is common in both modes of separation. ANP displays rapid equilibration of the separation materials and, in some cases it is possible to operate both separation modes simultaneously. This unique character helps in separating both hydrophobic and hydrophilic compounds in a single isocratic or gradient run. A number of silica hydride-based stationary phases have been studied because, it is possible to obtain reversed-phase and aqueous normal phase capabilities by varying the surface composition 
for a range of applications. The transition between ANP and RP is possible only with silica hydride stationary phases by the use of water in the mobile phase.

The HILIC separation mode cannot undergo this transition. Therefore it can only separate polar compounds. This unique property of ANP makes it different from HILIC in which only polar compounds are separated [1-4].

\section{Resolution and Column Efficiency}

Resolution is one of the main factors that define the efficiency of HPLC columns. It describes how well separated two peaks are from each other.

$$
\mathrm{R}=1.18 \Delta \mathrm{t}_{\mathrm{R}} / \mathrm{W}_{1}+\mathrm{W}_{2}
$$

Where $\Delta \mathrm{t}_{\mathrm{R}}$ is the difference in retention times between two peaks and $\mathrm{W}_{1}$ and $\mathrm{W}_{2}$ are the widths of the two peaks.

The other factors on which HPLC separation and efficiency depend are column selectivity $(\alpha)$, the sample capacity factor $\left(k^{\prime}\right)$, and column efficiency $(\mathrm{N})$.

Column selectivity is defined as

$$
\alpha=\left(\mathrm{t}_{\mathrm{R} 2}-\mathrm{t}_{0}\right) /\left(\mathrm{t}_{\mathrm{R} 1}-\mathrm{t}_{0}\right)
$$

Where $t_{R 2}$ is the retention time of the second peak, $t_{R 1}$ is the retention time of the first peak and $t_{0}$ is the retention time of the void volume. Separation is achievable for $\alpha>1$. The sample capacity factor is defined as

$$
\mathrm{k}^{\prime}=\left(\mathrm{t}_{\mathrm{R}}-\mathrm{t}_{0}\right) / \mathrm{t}_{0}
$$

Where $t_{R}$ is the retention time of a retained peak and $t_{0}$ is the retention time of the void volume. 
The column efficiency $(\mathrm{N})$ is based on the number and height of the theoretical plates, which can be determined by the equation

$$
\mathrm{N}=5.54\left(\mathrm{t}_{\mathrm{R}} / \mathrm{W}\right)^{2}
$$

Where $t_{R}$ is the retention time of a solute and $W$ is the width of the peak.

The height equivalent to a theoretical plate (HETP) can be calculated by using the Van Deemter equation

$$
\mathrm{HETP}=\mathrm{L} / \mathrm{N}
$$

Where $\mathrm{L}$ is the column length and $\mathrm{N}$ is the number of theoretical plates. A low HETP value represents high column efficiency $[1,5]$.

\section{Column Material}

The solid supports that can be used for surface modification are polymers, titania, alumina, zirconia, thoria, and silica. Silica is the most widely used solid support in liquid chromatography because it is inexpensive and is commercially available in almost any particle size. It has a very high mechanical stability, is very rigid, and can resist swelling. It also provides high efficiency and comes in different pore sizes which make it easy to modify in various ways.

Although silica has been one of the dominant choices as a solid support for stationary phases, numerous attempts have been made to find an alternative material to overcome some of the deficiencies associated with it. The major issues associated with silica while using it as a stationary phase are $\mathrm{pH}$ instability and its strong affinity for basic compounds. Silica has problems when measuring samples with a $\mathrm{pH}$ less than 2 or 
greater than 8 , as at a lower $\mathrm{pH}$ the stationary phase undergoes decomposition, while at higher $\mathrm{pH}$ it begins to dissolve. It is possible to operate columns at a higher $\mathrm{pH}$, provided the silica support is densely bonded, extensively endcapped, or has a longer chain column packing that allows the use of organic buffers as part of the mobile phase.

The silica surface can have different kinds of active groups. These groups are shown in Figure 1 [6]. The different active groups are siloxane, free silanols, geminal silanols, vicinal silanols, and bonded water. Each of these active groups has advantages and disadvantages depending on the type of analysis conducted. These active groups can help in the separation of some compounds and interfere with others. The functionality that is most widely used in the modification of silica surfaces is free silanol [6].

There are different types of silica that are available and are distinguished based on pore size, specific surface area, particle size, etc. The important factor to consider when choosing silica is the pore size, since it determines the interacting surface of the stationary phase. In general, larger pore size silica is useful for the analysis of large-sized or macromolecules, whereas smaller pore size silica is useful for the analysis of small molecules. It is possible to operate silica at different $\mathrm{pH}$ values, under high pressures, and in both aqueous and organic mobile phases used during the analysis [7]. 
1) Siloxane

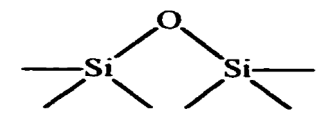

2) Free Silanols

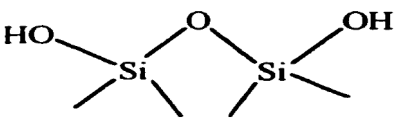

3) Geminol Silanol

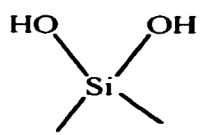

4) Vicinol Silanol

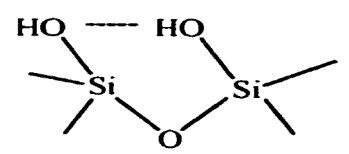

5) Bound Water

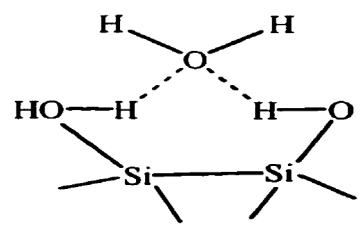

Figure 1. Active groups on a silica surface.

The type of silica used for this research was Astrosil type B silica, which has a narrow pore size distribution (100 $⿱$ ) $)$. It is not possible to convert all the silanol groups during bonded phase reactions due to steric effects. These unreacted silanol groups can cause problems like peak tailing and loss of chromatographic resolution. These groups can also interact with analytes via hydrogen bonding [7]. The main goal of this research was to first convert silica to silica hydride via a silanization reaction, and then cap the unreacted silanol groups with a methyl group and characterize it through chromatograpic techniques. 


\section{E. Silica Surface Modification}

Silica is the most extensively used stationary phase material, but it cannot be used in its native form, because of its limited $\mathrm{pH}$ stability and peak tailing when analyzing basic compounds. The prolonged exposure to strongly acidic and basic aqueous mobile phases can deteriorate the silica surface. Making modifications to the surface of silica can be advantageous in terms of $\mathrm{pH}$ stability, as well as peak tailing, and allows the silica support to be used with aqueous solutions for a longer period of time. Silanol groups on the silica surface react with bonding groups to form the bonded phase [8]. Silica surfaces can be modified using several methods. Some examples are:

\section{Esterification}

The reaction is achieved between a silanol group and an alcohol group. The reaction can be written as

$$
\mathrm{Si}-\mathrm{OH}+\mathrm{R}-\mathrm{OH} \text {---> Si-OR + } \mathrm{H}_{2} \mathrm{O}
$$

The link between the organic moiety and the silica surface is silicon-oxygen-carbon ( $\mathrm{Si}$ O-C). This method is very simple and the bonded phase is thermally stable. The drawback of this modification is that the bonded phase is very unstable in the presence of water. Therefore it is not possible to use stationary phases based on this modification for many HPLC applications with aqueous mobile phases [8]. 


\section{Organosilanization}

The majority of commercially available columns use the organosilanization method for silica surface modification. The reaction involves an organosilane reagent with a halide, methoxy, or ethoxy group and the silica surface. The organosilane reagent is covalently bonded to the silica surface and the reaction can be used to produce monomeric or polymeric stationary phases, depending on the type of silane used. An organosilane reagent with three reactive groups forms a multilayered polymeric stationary phase due to crosslinking between the adjacent organosilane groups. The general disadvantage of organosilanization is that an acid by-product is formed during the reaction which can hydrolyze the $\mathrm{Si}-\mathrm{O}-\mathrm{Si}-\mathrm{C}$ bonds. Monomeric organosilane stationary phases have poor hydrolytic stability under acidic and basic conditions [9-10].

The organosilanization reaction can be written as

$$
\begin{gathered}
\mathrm{Si}-\mathrm{OH}+\mathrm{X}-\mathrm{Si} \mathrm{R}_{2}{ }_{2} \mathrm{R}--->\mathrm{Si}-\mathrm{O}-\mathrm{Si} \mathrm{R}_{2}{ }_{2} \mathrm{R}+\mathrm{HX} \\
\text { X-halide and R-alkyl } \\
\mathrm{Si-OH}+\mathrm{X}_{3}-\mathrm{Si}-\mathrm{R} \text {----> Si-O-Si-R + 3HX }
\end{gathered}
$$

\section{Chlorination Reaction Followed by a Reaction with Grignard Reagents or an Organolithium Compounds}

In this two step reaction, the first step is carried out by reacting silica with thionyl chloride to form an unstable chlorinated intermediate. In the second step the chlorinated intermediate reacts with an organometallic compound leading to the formation of a $\mathrm{Si}-\mathrm{C}$ linkage. The disadvantages of this reaction are that the byproduct metal salt which is 
formed in the second step is very difficult to separate from the original product, and the chlorinated intermediate is very unstable, hence the reaction has to be carried out in dry environment [8-9].

The reaction can be written as:

$$
\begin{gathered}
\mathrm{Si}-\mathrm{OH}+\mathrm{SOCl}_{2}---->\mathrm{Si}-\mathrm{Cl}+\mathrm{SO}_{2}+\mathrm{HCl} \\
\mathrm{Si}-\mathrm{Cl}+\mathrm{BrMgR}---->\mathrm{Si}-\mathrm{R}+\mathrm{MgClBr} \\
\text { or } \\
\mathrm{Si}-\mathrm{Cl}+\mathrm{Li}-\mathrm{R}----->\mathrm{Si}-\mathrm{R}+\mathrm{Li}-\mathrm{Cl}
\end{gathered}
$$

\section{Silanization/Hydrosilation}

The recent approach for the modification of silica surfaces involves the use of triethoxysilane (TES) in a reaction called silanization, which is followed by a hydrosilation reaction to attach an alkyl group to the silica surface. The silanization reaction involves the formation of a stable hydride monolayer by the reaction of silica with TES in the presence of an acid catalyst. The hydride intermediate formed can be stored for longer periods of time, as it is stable in air or water without degradation. The reaction has been reported to yield a 95\% surface coverage with a nonpolar $\mathrm{Si}-\mathrm{H}$ monolayer [11]. 


\section{a. Silanization}

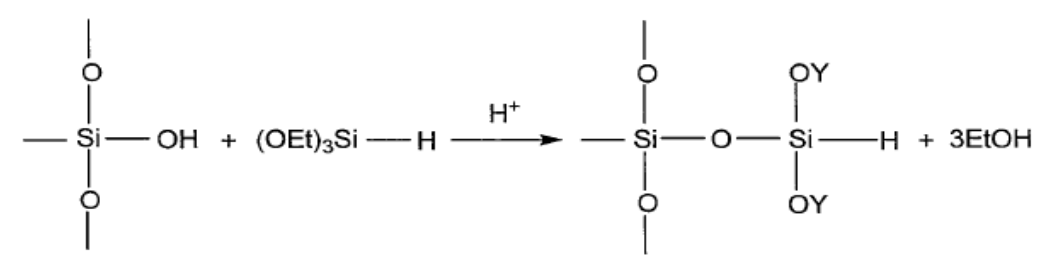

b. Hydrosilation

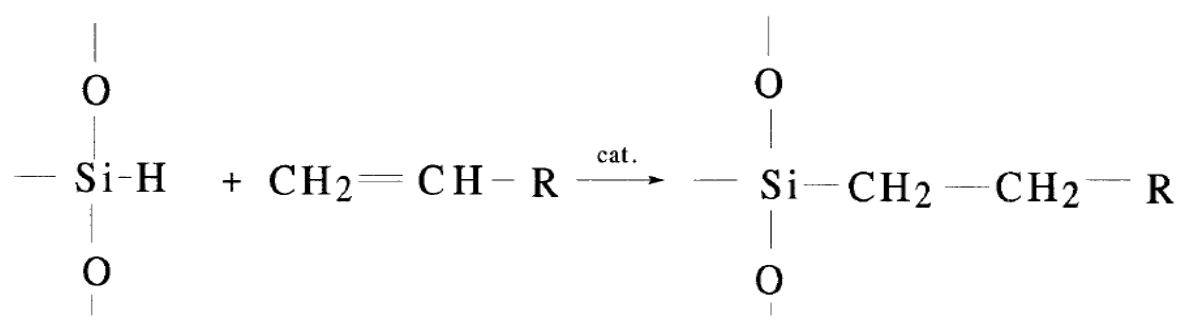

cat. $=$ catalyst, typically hexachloroplatinic acid

The attachment of an organic group at the silica hydride surface to form a Si-C bond occurs in the hydrosilation step. The reaction is facilitated in the presence of a transition metal complex catalyst, usually Speier's catalyst, a 2-propanol solution of hexachloroplatinic acid. The drawback of this reaction is that the transition step while attaching an olefin to the silica hydride forms a complex with the catalyst. The use of a transition metal complex catalyst forms the intermediate that can be of very large in size. This might result in a lower yield of organic group on the surface; even though having a smaller size olefin in comparison to the organosilane species in the conventional 
organosilanization bonding methods. The other problem is that the catalyst can undergo reduction by itself. The silica hydride and metal complex catalyst react together and form Pt (0) deposition on the surface. The presence of the metal on the surface affects the chromatographic performance. The use of free radical initiation instead of a transition metal complex might solve the problem. But hydrosilation via a metal complex has been proven to be successful on silica hydride, and limited studies have been done on free radical initiation [12]. The formation of the $\mathrm{Si}-\mathrm{C}$ bond at the surface seems to be more thermodynamically and hydrolytically stable than the commercially available stationary phases that are formed after an organosilation reaction.

An important advantage of hydrosilation method is that the silica hydride intermediate can be used by itself as a stationary phase for many chromatographic applications. It is possible to use silica hydride columns in reversed-phase conditions, organic normal phase conditions, and aqueous normal phase conditions. The research focused on the aqueous normal phase and reversed-phase conditions using the endcapped Si-H stationary phase and comparing it with a C8 silica hydride column. As shown in Figure 2 [2], in reversed-phase chromatography the composition of the mobile phase water/acetonitrile varies from $0 \%$ to $100 \%$ of organic constituents, which decreases the retention of hydrophobic analytes as the composition of less polar organic solvent is increased [2]. 


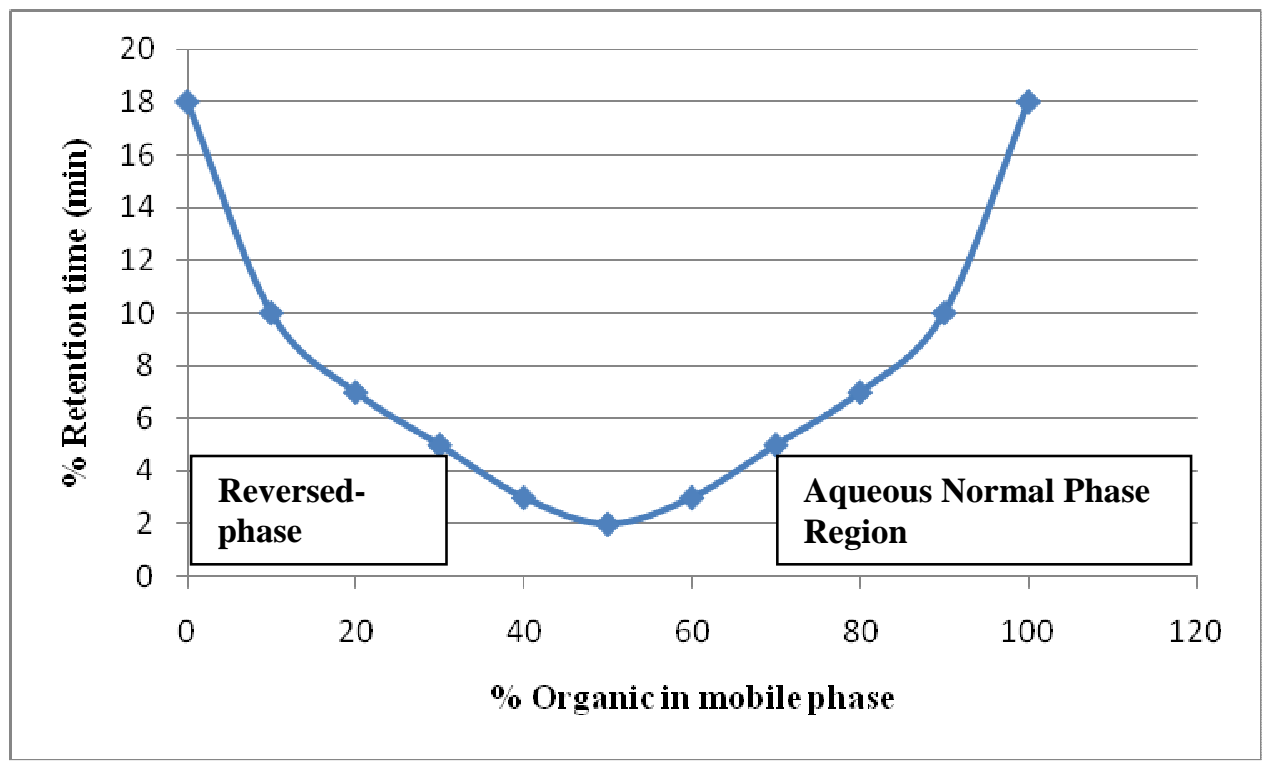

Figure 2. Retention map for RP and ANP region on a Si-H based stationary phase.

The goal for this research was to use a silica hydride intermediate and endcap the surface with trimethylchlorosilane (TMCS) and hexamethyldisilazane (HMDS). The endcapping was performed for the remaining unreacted silanol groups which were not converted to $\mathrm{Si}-\mathrm{H}$ groups. This enhanced the column efficiency by minimizing the amount of silanol groups on the silica surface. Endcapping is defined as the secondary derivatization reaction that can be used to shield and reduce silanol groups on a modified silica surface $[9,13-14]$. 


\section{F. Endcapped Silica Hydride Stationary Phase}

Endcapping can be done using polar reagents with an amide group or an alcohol group, or using nonpolar reagents with a methyl group depending on the type of separation that needs to be achieved. The use of an organosilane reagent replaces many of the unreacted silanol groups on silica and also increases the hydrophobicity of the stationary phase surface. The organosilane reagent reacts with only $50 \%$ of the available silanol groups and the remaining groups can cause problems by interacting with the analyte molecules. Endcapping overcomes problems with the interactions between the analyte and silanol groups by converting $\mathrm{Si}-\mathrm{OH}$ groups to $\mathrm{Si}-\mathrm{O}-\mathrm{Si}-\mathrm{C}$ groups. Endcapping of the silica hydride stationary phase uses a 1:1 molar ratio of trimethylchlorosilane (TMCS) and hexamethyldisilazane (HMDS). TMCS-HMDS eliminates the few remaining unreacted and accessible silanols on the silica hydride intermediate which increases hydrophobicity of the surface. It is these strongly adsorbing sites on the surface of silica that are the targets for any endcapping [15].

For this research, Astrosil silica was first modified to silica hydride using triethoxysilane (TES), a silanization reaction. Then the silica hydride was endcapped with a 1:1 molar ratio of TMCS and HMDS to minimize the amount of silanol groups on the silica surface. The endcapping of silica hydride increased the hydrophobicity of the stationary phase which in turn was useful for the separations of nonpolar compounds. The results of endcapped $\mathrm{Si}-\mathrm{H}$ were compared with a $\mathrm{Si}-\mathrm{H} \mathrm{C} 8$ column for chromatographic separations under aqueous normal phase and reversed-phase conditions. 
The obtained results help to understand the polarity difference between the two columns and its applications. These studies determine whether endcapping is sufficient after silanization or further modification is required for the separation of nonpolar compounds.

\section{G. Advantages of Using Hydride-based Stationary Phases}

Silica hydride-based columns are desirable because of their high mechanical strength, high column efficiency, and ease of fabrication. It is possible to develop several bonded phases via silica derivatization with different compounds having different functional groups. Silanol groups are the main reaction sites for silica and modification to a silica matrix can be measured using magic-angle-spinning (MAS) ${ }^{29} \mathrm{Si}$ NMR and diffuse reflectance infrared Fourier transform spectroscopy [4].

Acidic silanol groups interact with basic analytes which leads to poor resolution, and peak tailing. Metals such as $\mathrm{Fe}, \mathrm{Al}$, and $\mathrm{Zn}$ which are found in silica can also contribute to peak tailing and retention properties during separation. Silica that is highly acidic and contains lower amounts of metals is a better starting material for stationary phase synthesis [4].

A comparison of the chemical composition between silica and silica hydride is shown in Figure 3 [2]. An ordinary silica surface has silanol groups ( $\mathrm{Si}-\mathrm{OH})$ while the hydride silica surface has $\mathrm{Si}-\mathrm{H}$ groups. The fundamental difference between the two materials leads to differences in their surface properties, which are responsible for the 
significant differences in chromatographic separation between the two surfaces. Silanol groups are highly polar while the $\mathrm{Si}-\mathrm{H}$ is weakly hydrophobic, and it has also been proved that silica hydride columns can be used for a wide range of applications [4].

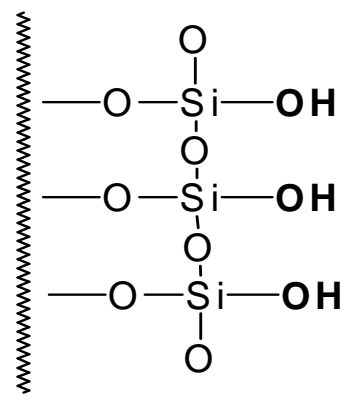

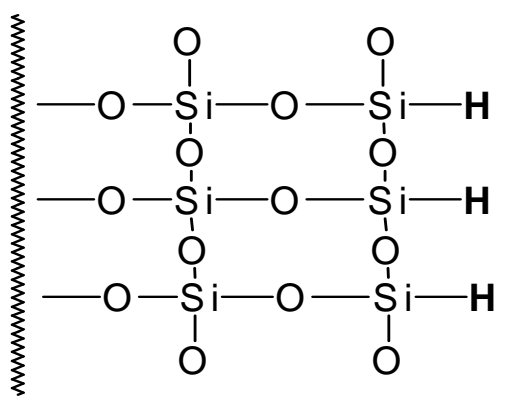

Figure 3. Idealized structures of a normal silica surface (left) and silica hydride surface (right).

It is possible to synthesize silica hydride in two ways, one with the use of thionyl chloride to form an $\mathrm{Si}-\mathrm{Cl}$ intermediate. The main drawback to this process is that $\mathrm{Si}-\mathrm{Cl}$ is very sensitive to moisture and the reaction is easily reversible once exposed. Another way of synthesizing silica hydride is via a silanization reaction. The process is done by the condensation reaction between silanols and TES. This is a single step reaction and is not sensitive to water as it uses an aqueous acid as a catalyst. Previous work has proven that it is possible to get up to $95 \%$ silica hydride surface under carefully controlled synthetic conditions [2, 4]. 


\section{H. Characterization Techniques}

Diffuse Reflectance Fourier Transform Infrared Spectroscopy, hydrophobicity, silanophilicity tests, and high performance liquid chromatography were used to characterize the endcapped stationary phase and the results were compared with a $\mathrm{C} 8$ silica hydride column.

\section{Diffuse Reflectance Fourier Transform Infrared Spectroscopy}

The spectroscopic characterization of the bonded material is done by using diffuse reflectance Fourier transform infrared spectroscopy (DRIFT), which determines the presence of organic moieties or functional groups on any silica surface including silica hydride. The measurement gives qualitative information about the structure of the molecule, and the fingerprint region of the molecule is often used to distinguish between one molecular structure and another. The Fourier transform process averages the signal, which increases the sensitivity of the measurement, and thus allows for the characterization of samples with weak absorption bands. DRIFT, like other infrared methods, measures molecular vibrations. The infrared region is divided into near, mid, and far regions with a frequency ranging from $12,800 \mathrm{~cm}^{-1}$ to $10 \mathrm{~cm}^{-1}$. The mid-infrared region ranges from $4000 \mathrm{~cm}^{-1}$ to $200 \mathrm{~cm}^{-1}$ is used to obtain structural information of the molecules. The molecules undergo vibrational or rotational motions upon absorption of infrared radiation which changes the dipole moment of the molecule. The molecules absorb radiation when the natural vibrational frequency of the molecule matches the frequency of the incident radiation. The radiation on the surface undergoes partial 
reflectance and partial transmittance and the light absorbed from the material undergoes reflectance and transmittance. DRIFT is used in this research to obtain specific structural information of the starting material as well as the synthesized product, which can be used to confirm the success of a specific reaction [1-2].

\section{Neue Test}

Neue test is used to determine the hydrophobicity and silanophilicity of a stationary phase material. The physico-chemical properties (hydrophobicity and silanophilicity) of the columns are measured at $\mathrm{pH}$ 7. The Neue test is described below.

\section{a. Hydrophobicity Parameter (u)}

The hydrophobicity test is used to determine the retention of nonpolar compounds; the greater the value of hydrophobicity of the column the greater the retention of nonpolar solutes.

The hydrophobicity parameter(u) is calculated as follows:

$$
\ln (\mathrm{k})=\mathrm{u} * \mathrm{~V}-3.068
$$

where $\mathrm{V}=$ the molar volume of the analyte (acenaphthene or naphthalene).

$$
\mathrm{k}=\left(\mathrm{t}_{\mathrm{r}}-\mathrm{t}_{0}\right) / \mathrm{t}_{0}
$$

$\mathrm{k}=$ retention factor and $\mathrm{t}_{0}=$ retention time of the void volume . 


\section{b. Silanophilic Interaction Parameter (S)}

The silanophilic test is used to measure strong polar/adsorptive interactions with the surface. The lower the value from the silanophilic test the lower the surface adsorption, which results in better peak shape for polar compounds. The compounds used for this test are amitryptiline, acenaphthene, and uracil.

The silanophilic interaction parameter(S) is calculated as follows:

$$
\begin{gathered}
\mathrm{S}=\ln \mathrm{k}_{\text {amitryptiline }}-0.7124 * 1 \operatorname{lnk}_{\text {acenaphthene }}+1.9748 \\
\mathrm{k}=\left(\mathrm{t}_{\mathrm{r}}-\mathrm{t}_{0}\right) / \mathrm{t}_{0}
\end{gathered}
$$

$\mathrm{k}=$ retention factor and $\mathrm{t}_{0}=$ retention time of the void volume [16].

\section{Elemental Analysis}

The quantitative micro-combustion method determines the amount of compound bonded to the silica surface. The measurement is based on a high temperature oxidation of the organic compounds by converting the elements of interest to gaseous molecules. The percentage carbon obtained indicates the amount of organic moiety that has been successfully attached on the silica surface [17].

\section{High Performance Liquid Chromatography}

As shown in Figure 4 [18] the concept of any chromatographic separation technique is that the sample mixture is injected onto the packed analytical column and the mobile phase is then pumped through the column. This carries the sample mixture along 
the stationary phase. Different compound mixtures have different interactions with the stationary phase and the mobile phase based on their properties. The separation is mainly based on their interaction towards the stationary phase or mobile phase. The compounds which are separated eventually elute from the column and are detected using an appropriate measurement apparatus. Different detectors are chosen based on the sample properties and the technique can be used for a large variety of mixtures.

There are two possible elution modes: the isocratic separation mode and the gradient separation mode. In isocratic elution the mobile phase composition is held constant throughout the separation, whereas in gradient elution the composition is changed throughout the process. It is possible that the gradient can be step or linear based on the mixture to be analyzed. Gradient elution is often used in separating biological samples of high molecular weight or to separate complex mixtures. The one disadvantage of using the gradient mode is that it shows prominent baseline drift for some detectors as the gradient proceeds. 


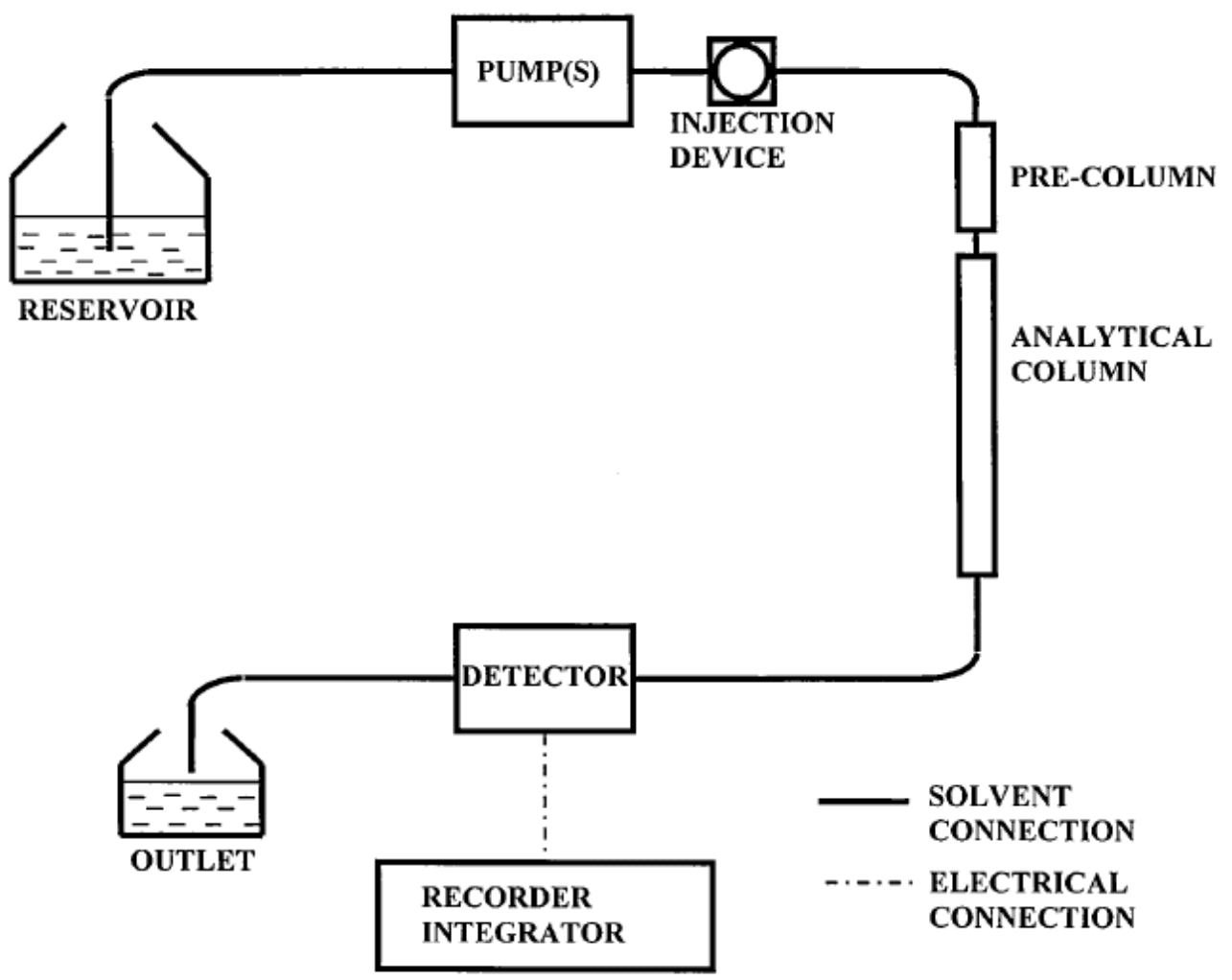

Figure 4. Schematic diagram of HPLC system.

The detectors also play a very important role in the HPLC system. They are chosen based on the sample characteristics and the kind of analysis. Some of the detectors available are UV, fluorescence, refractive index, charged aerosol detector, evaporative light scattering, and mass spectrometry. The mass spectrometer is the most sensitive detector and is extensively used in almost all industries. It is considered the universal detector as it provides molecular information of the sample. UV detectors are commonly used, as they are less expensive and used for the compounds that have a chromophore to absorb the UV radiation. The fluorescence detector is used for the 
compounds which have a flurophore, and refractive index detectors are used for compounds which cannot be detected by UV. The drawback of the refractive index detector is that it cannot be used for gradient elution conditions. Charged aerosol detectors are used for compounds which are easily evaporated and are much more sensitive than UV detectors $[4,18]$.

\section{a. Evaporative Light Scattering Detector}

The evaporative light scattering detector (ELSD) is one of the detectors used for this research. This detector is used for compounds which cannot be analyzed using UV, compounds that do not have a chromophore and also it is gradient compatible. Therefore it can be used as an alternative to the refractive index detector. The eluent stream passes through the nebulizer into the evaporation chamber. The nebulizer transforms the column eluent into small droplets by the use of the inert gas nitrogen. Then the droplet is allowed to evaporate to form fine particles suspended in the atomizing gas. The suspended particles pass through a laser beam and, based on the light scattering of the incident beam, the particles are detected by means of an optical fiber.

The three simple steps on which the ELSD operates are nebulization, evaporation, and detection. During nebulization, the column eluent passes through a nitrogen gas spray needle and forms small droplets of aerosols. The aerosols then pass through a heated drift tube, where it is evaporated to form a fine uniform mist of sample particles in a solvent vapor. The evaporated particles in the solvent vapor pass through a cell and scatter the laser light beam. The scattered light is then detected with the help of a 
photodiode generating a signal. ELSD is useful for the detection of amino acids because a traditional UV detector is insensitive to these compounds since most do not have a chromophore.

ELSD requires that a part of the aerosol be diverted to waste in order to measure effectively under high HPLC flow rates. The earliest design used a nebulization chamber with a restricted flow caused by a sharp turn, in which only smaller particles would successfully pass through and move towards the detection cell. The larger particles fail to pass and are directed toward the waste line. This design is appropriate for high flow rates but has difficulty in evaporating the mobile phase. Therefore, most of the sample is sent to waste which in turn compromises the detector sensitivity. To overcome the drawbacks, a plate impactor is designed. Larger particles hit the plate, condense, and go to waste whereas the smaller particles make it through the annular spaces of the impactor. The plate impactor can be either on or off and there are no settings to moderate the flow rates or to moderate the evaporation of mobile phases.

The SofTA Model 400 ELSD detector is used for this research. The use of thermo-split technology provides a smooth variation of an aerosol cloud over a wide range of conditions. The split ratio of the aerosol can be controlled by an aerosol splitter that combines a gentle bend with temperature controlled walls. It is easy to evaporate mobile phases with high organic content by heating the walls of the chamber. The increased temperature results in the rapid evaporation of solvent which allows all the particles to negotiate the bend. It is difficult to evaporate mobile phases with high aqueous solvents or at high flow rates; therefore the walls of the chamber are cooled, and 
some fraction of the eluent is sent to waste. These experimental conditions result in part of the aerosol condensing to form larger particles which fail to negotiate the bend, and are sent to waste. The one advantage of using this system is the possibility of controlling the split ratio in a smooth analog fashion by varying the temperature of the spray chamber. The temperature can be varied from $0^{\circ} \mathrm{C}$ to $80^{\circ} \mathrm{C}$ in $1^{\circ} \mathrm{C}$ increments and is very easy to operate with a user-friendly touch screen that allows online monitoring as well. The schematic representation of the thermo-split chamber is shown in Figure 5. The top diagram represents the heated surface and bottom diagram represents the cooled surface where the solvent can be condensed and passed to the drain.

The advantages of using an evaporative light scattering detector are that it is a universal detector which can be used for all samples that are less volatile than the mobile phase. Qualitative analysis of a sample is more accurate than the other commonly used detectors. It does not require any sample preparation which eliminates the sample derivatization step. It has a very high sensitivity which can detect samples in the nanogram range. The baseline is relatively stable even with a rapidly changing gradient. The cost of operating the instrument is cheaper when compared to a mass spectrometer. The disadvantage of using the ELSD detector is that it cannot be used for quantitative measurements because the concentration of the sample is non-linear with respect to the peak area. The light scattering measurement is a function of particle size rather than the concentration of the sample. ELSD can be used on samples which are less volatile than the mobile phase; therefore, only volatile mobile phase modifiers can be used. The use of non-volatile mobile phase modifiers leads to detector noise [4, 19-20]. 


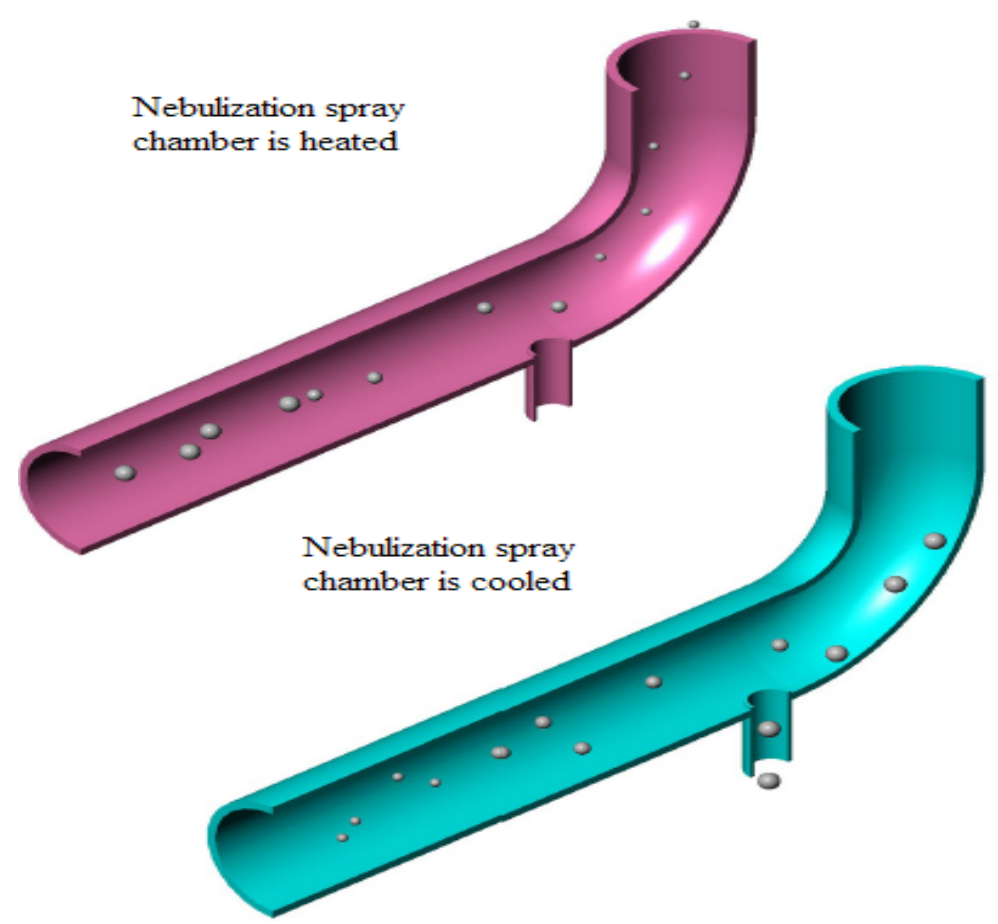

Figure 5. Schematic diagram of nebulization spray chamber.

\section{Goals}

The initial step was to synthesize a silica hydride stationary phase using Astrosil silica and TES via a silanization reaction. Then characterization of the silica hydride material was done using DRIFT which confirms the success of the reaction. The silica hydride material was endcapped using TMCS and HMDS for the unreacted silanol groups to increase the efficiency and peak symmetry of the column. The endcapped silica hydride material was again characterized using DRIFT to confirm the success of the reaction.

The initial objective of this research was to synthesize an endcapped silica hydride stationary phase followed by spectroscopic characterization to confirm the endcapped silica hydride stationary phase formation. The final goal of this research was 
to determine the ability of the endcapped silica hydride column to operate in the aqueous normal phase mode for selected small molecules such as amino acids, weak organic acids, and vitamins. The study was done using the retention of analytes based on hydrophilicity in ANP chromatography and hydrophobicity in RP high performance liquid chromatography. The same experiments were repeated with a $\mathrm{C} 8 \mathrm{Si}-\mathrm{H}$ column under the same conditions and using the same instrument. The results from $\mathrm{C} 8$ were compared with the results of the endcapped $\mathrm{Si}-\mathrm{H}$ column to determine the separation capabilities of the endcapped column in comparison with the C8 Si-H column. 


\title{
II. EXPERIMENTAL
}

\author{
A. Materials
}

\section{Chemicals}

The chemicals and compounds used for this research in the preparation and characterization of stationary phases are listed in this section.

Table 1. Type of silica used for the silica hydride synthesis

\begin{tabular}{|c|c|c|c|}
\hline Type of silica & Particle size & Pore size & Lot number \\
\hline Astrosil spherical & $4.2 \mu \mathrm{m}$ & $108 \AA$ & $7-122 \mathrm{~F} 2$ \\
silica & & & \\
\hline
\end{tabular}


Table 2. Chemicals used for synthesis and characterization

\begin{tabular}{|c|c|}
\hline Chemical Name & Supplier Name \\
\hline Acetonitrile & Fisher chemical, Pittsburg, PA \\
\hline Trifluoroacetic acid & Thermoscientific, Barrington, IL \\
\hline Formic acid & Thermoscientific, Barrington, IL \\
\hline Deionized water & Millipore \\
\hline Dioxane & Fisher chemical, Pittsburg, PA \\
\hline Triethoxysilane & Aldrich Chemical Co., Milwaukee, WI \\
\hline Toluene & Fisher chemical, Pittsburg, PA \\
\hline Diethyl ether & Fisher chemical, Pittsburg, PA \\
\hline Trimethylchlorosilane & Sigma Aldrich, St Louis, MO \\
\hline Hexamethyldisilazane & Sigma Aldrich, St Louis, MO \\
\hline
\end{tabular}




\section{Compounds Used for Chromatographic Characterization}

Table 3. Chemicals used for hydrophobicity and silanophilicity test

\begin{tabular}{|c|c|}
\hline Chemical Name & Supplier Name \\
\hline Sodium monobasic phosphate & Spectrum, Newark, CA \\
\hline Sodium dibasic phosphate & Spectrum, Newark, CA \\
\hline Methanol & J. T. Baker Chemical Co., Phillipsburg, NJ \\
\hline Uracil & Matheson Coleman \& Bell, Cincinatti, \\
& Ohio Lot number=UX55-5985 \\
\hline Naphthalene & J. T. Baker Chemical Co., Phillipsburg, NJ \\
\hline Acenaphthene & J. T. Baker Chemical Co., Phillipsburg, NJ, \\
& Lot number=A006 \\
\hline Amitryptiline hydrochloride & J. T. Baker Chemical Co., Phillipsburg, NJ \\
\hline
\end{tabular}


Table 4. Compounds used for analysis

\begin{tabular}{|c|c|}
\hline Chemical Name & Supplier Name \\
\hline Phenylalanine & J.T.Baker, Phillipsburg, NJ \\
\hline Niacinic acid & Merck \& Co., Inc, Rahway, NJ \\
\hline Citric acid & Mallinckrodt, St Louis, MO 63147 \\
\hline Lisinopril & Sigma-Aldrich, St Louis, MO \\
\hline Raloxifene & Sigma-Aldrich, St Louis, MO \\
\hline Dimethylthiourea & Sigma-Aldrich, St Louis, MO \\
\hline Lipoic acid & Sigma-Aldrich, St Louis, MO \\
\hline Triphenylphosphine & Sigma-Aldrich, St Louis, MO \\
\hline Melamine & Sigma-Aldrich, St Louis, MO \\
\hline
\end{tabular}




\section{Structures of the Compounds Used for Neue Test}

The structures of the compounds used for Neue test are shown in

Figure 6 [21-24]

Uracil

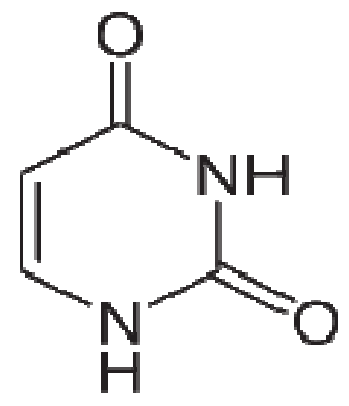

Naphthalene

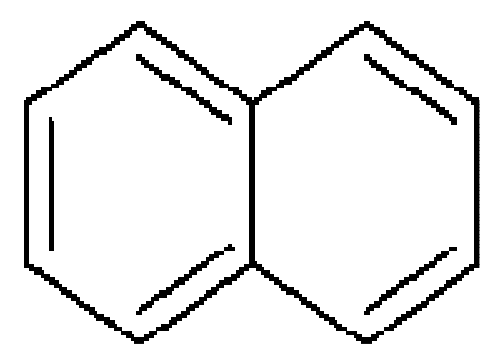

Acenaphthene

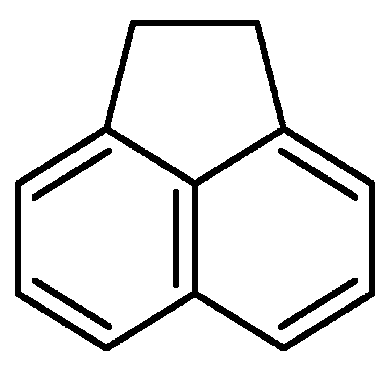

Amitriptyline hydrochloride

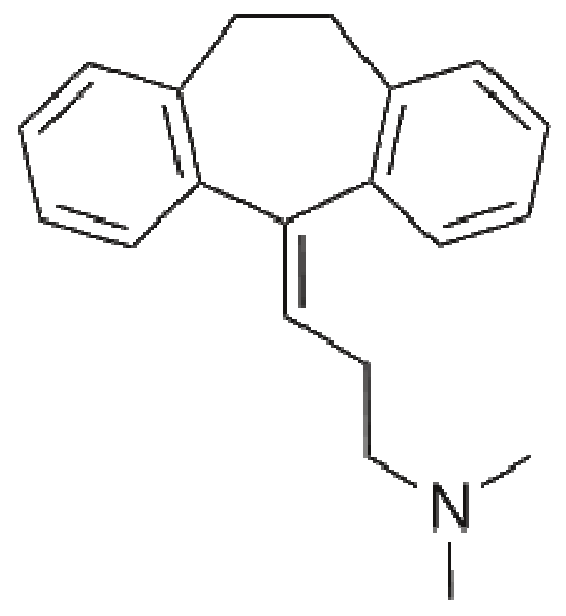

Figure 6. Structures of Neue test compounds. 


\section{Structures of Compounds Used for Chromatographic}

\section{Characterization}

The structures of the compounds used for chromatographic characterization are shown in Figure 7 [25-33].

\section{Phenylalanine}

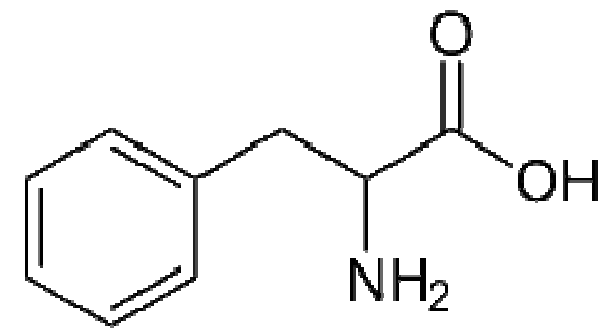

Melamine

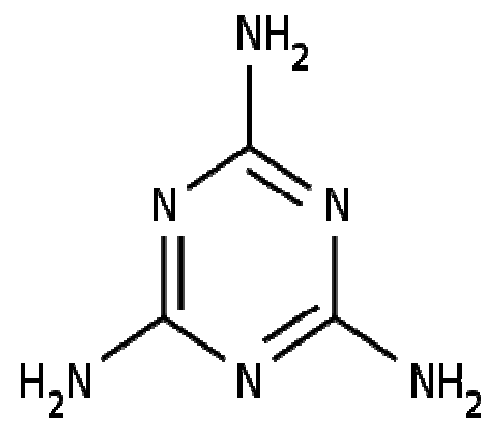

\section{Lisinopril}<smiles>NCCCC[C@H](N[C@@H](CCc1ccccc1)C(=O)O)C(=O)N1CCC[C@H]1C(=O)O</smiles> 
Niacinic acid

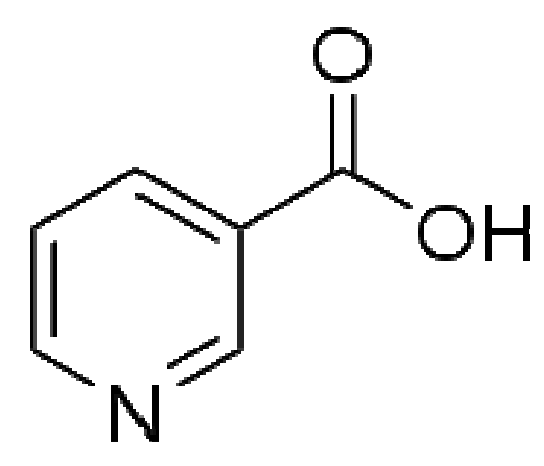

Raloxifene

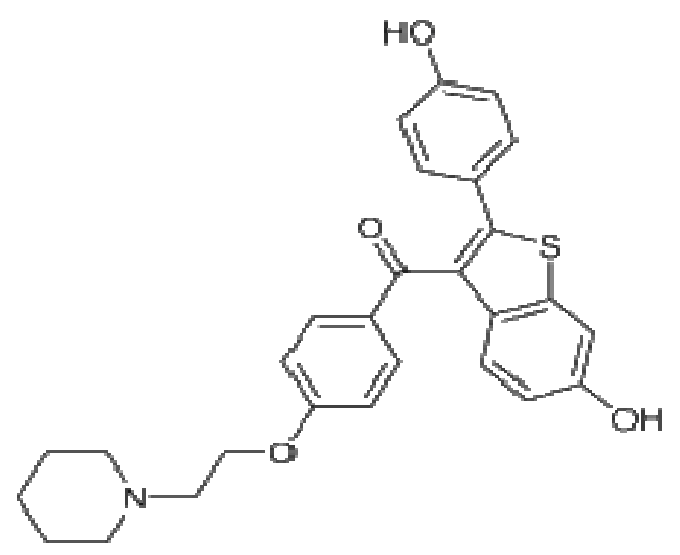

Dimethylthiourea

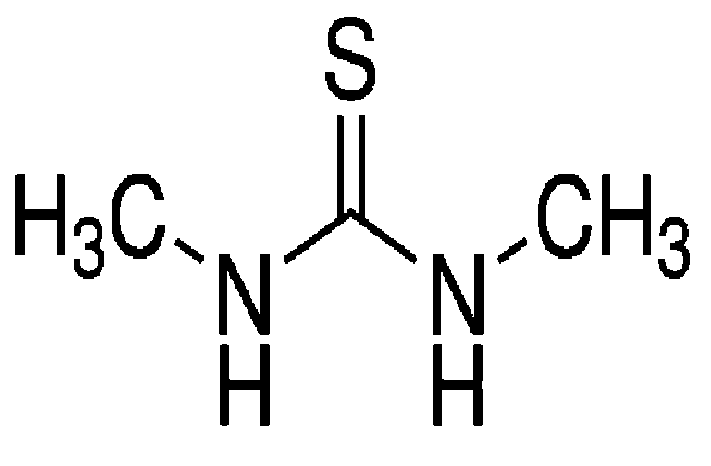

Citric acid

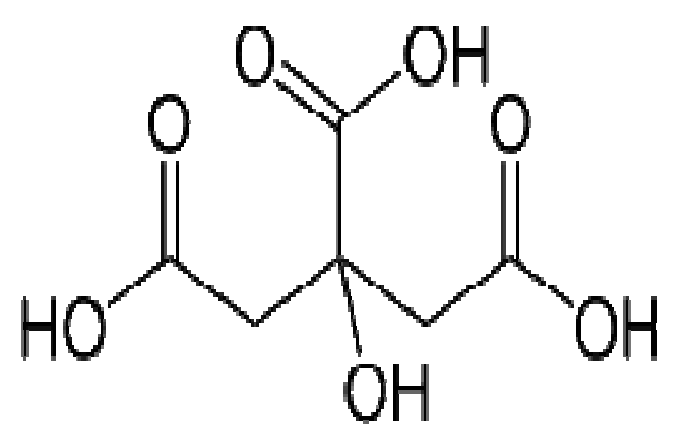




\section{Lipoic acid}

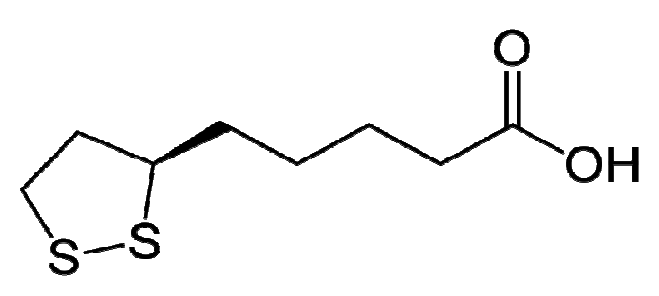

Triphenylphosphine

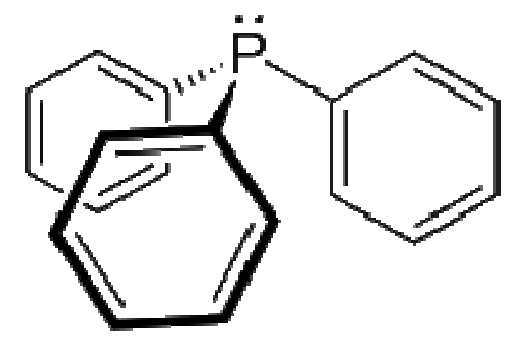

Figure 7. Structures of compounds used for chromatographic Characterization.

\section{B. Synthetic Procedures}

\section{Synthesis of Silica Hydride via Silanization}

All the glassware required for the silica hydride synthesis was dried overnight in an oven at $110^{\circ} \mathrm{C}$. The Astrosil silica was also dried overnight in a vacuum oven at $120^{\circ} \mathrm{C}$ prior to synthesis. The TES mixture was prepared by adding $90.15 \mathrm{~mL}$ of dioxane to a round bottom flask, attached to an addition funnel and a stopper and then added $20.85 \mathrm{~mL}$ of TES in an argon atmosphere. On a heating mantel and a magnetic stir plate, a 3-necked $1000 \mathrm{~mL}$ round bottom flask fitted with a water condenser and a drying tube, a magnetic stir bar, a thermometer to monitor the temperature during the synthesis, a glass stopper, and an addition funnel to add solvent were set up. To the round bottom 
flask, $300 \mathrm{~mL}$ of dioxane was added through an addition funnel followed by the silica, which was removed from the vacuum oven. The addition funnel was rinsed off with 300 $\mathrm{mL}$ dioxane to minimize the loss of silica and then $7.35 \mathrm{~mL}$ of $2.3 \mathrm{M} \mathrm{HCl}$ was added, and then the reaction mixture was heated to $70^{\circ} \mathrm{C}$. When the reaction mixture reached $70^{\circ} \mathrm{C}$, the TES mixture was added drop-wise through the addition funnel for 20 to $30 \mathrm{~min}$ with constant stirring. After adding all of the TES mixture, the temperature was raised to $90^{\circ} \mathrm{C}$ and the mixture was allowed to reflux for $90 \mathrm{~min}$. After $90 \mathrm{~min}$, the reaction mixture was cooled down to room temperature and was transferred to a medium sized filter crucible, attached to a filtering flask. The mixture was filtered using vacuum suction and then the solid silica was collected in the filter crucible. It was then washed twice with dioxane, three times with toluene, and three times with diethyl ether, using 50 $\mathrm{mL}$ of each solvent each time. The final silica hydride product was dried overnight at room temperature by placing the beaker in the hood to evaporate the ether used during washing cycles. The final product was then dried overnight in the vacuum oven at $110^{\circ} \mathrm{C}$.

\section{Endcapping of Silica Hydride}

The experimental setup was identical to the one used for silica hydride synthesis. The set-up involved 3 necked round bottomed flasks equipped with a water condenser and drying tube, a thermometer, a glass stopper, a magnetic stir bar, a magnetic stirrer, and a heating mantle. Like silanization, prior to endcapping, all the required glassware was dried overnight in the oven at $110^{\circ} \mathrm{C}$. The Astrosil silica hydride was kept in the 
vacuum oven overnight at $100^{\circ} \mathrm{C}$ prior to synthesis. Endcapping was carried out by adding silica hydride, trimethylchlorosilane, and hexamethyldisilazane to toluene, and the reaction mixture was heated to $60^{\circ} \mathrm{C}$. The reaction mixture was heated and refluxed for $120 \mathrm{~min}$ at $100^{\circ} \mathrm{C}$. Upon completion of the reaction, the reaction mixture was cooled rapidly and the silica hydride was separated by filtration.

Endcapping procedure: A 3-necked flask with a condenser was placed on the heating mantle which was set-up on a stir plate (used for stirring the reaction mixture) and $30 \mathrm{~mL}$ of toluene was added to the 3-necked flask and heated to $60^{\circ} \mathrm{C}$. While it was being heated, three other components were added to the toluene in the order listed:

1. $3 \mathrm{~g}$ of silica hydride

2. $3.38 \mathrm{~mL}$ of TMCS-TMCS trimethylchlorosilane $97.0 \%$

3. $5.67 \mathrm{~mL}$ of HMDS 1,1,1,3,3,3-hexamethyldisilazane $99.0 \%$

The reaction flask was flushed with argon and the reaction mixture was heated to $100^{\circ} \mathrm{C}$ and refluxed for $120 \mathrm{~min}$ for the reaction to complete. After the reaction period, the heating mantle was turned off and the mixture was cooled as rapidly as possible. However, removal of the reaction solution began within 5 min after completion of the procedure. The silica hydride was separated from the reaction solution by filtration using a filtering funnel (90M). It was then washed two times with dioxane, three times with toluene, and three times with diethyl ether, using $50 \mathrm{~mL}$ of each solvent each time. The final endcapped silica hydride product was dried overnight at room temperature by placing the beaker in the hood to evaporate the ether used during wash cycles. The finished product was dried overnight in a vacuum oven at $110^{\circ} \mathrm{C}$. 


\section{Instrumentation Procedures}

\section{Diffuse Reflectance Infrared Fourier Transform Spectroscopy}

Spectroscopic characterization was used to confirm the formation of $\mathrm{Si}-\mathrm{C}$ bonds after the endcapping process. An ATI Mattson Infinity Series FT-IR spectrophotometer with a Hewlett Packard FX-2 computer was used to collect the spectrum of Si-H and endcapped $\mathrm{Si}-\mathrm{H}$ surfaces. Prior to the sample scan, a measurement of pure $\mathrm{KBr}$ was taken as a reference. The sample was measured by using a 95:5 ratio of solid sample to IR-grade $\mathrm{KBr}$. The sample was ground finely using a pestle and placed in a sample cup 2 $\mathrm{mm}$ in diameter and $2 \mathrm{~mm}$ in depth, and the powder was leveled with a spatula. The sample cup was placed in the DRIFT sample holder and scanned. The sample was scanned 200 times at a resolution of $2 \mathrm{~cm}^{-1}$ over a range of $4000-450 \mathrm{~cm}^{-1}$ and normalized to $100 \%$ transmittance.

\section{Column Packing}

The stationary phase material after synthesis was packed in-house by a slurry method using a $\mathrm{CCl}_{4}$-methanol $(9: 1 \mathrm{v} / \mathrm{v})$ mixture. The dimensions of the stainless steel tubes used for packing the bonded phase material were $75 \times 4.6 \mathrm{~mm}$ i.d, which were provided by Alltech located at Deerfield of Illinois. A pneumatic pump, operated at 6000 psi pressure with HPLC grade methanol as the driving solvent, was used to pack the 
column. The column was held in the above condition for about 30 min before being removed.

\section{High Performance Liquid Chromatography}

The HPLC system used for the research was a SoftA Corporation Model 400 Evaporative Light Scattering Detector (ELSD) instrument equipped with an LDC Milton Roy CM400 binary pump system, a Rheodyne Model 7126 manual injector with a $20 \mu \mathrm{L}$ injection loop, and a Waters in-line degasser. The ELSD detector operated with the supply of nitrogen at 65 psi for nebulization of the column eluent. The ELSD used had a $670 \mathrm{~nm}$ laser diode and hermetically sealed photodiode for detection. The data collection system consisted of Chemstation software, an HP35900C multichannel interface, and an HP Deskjet $693 \mathrm{C}$ printer. The mobile phase was vacuum filtered using a $0.2 \mu \mathrm{m}$ Nylon 66 membrane (Alltech Associates, Inc. Deerfield, IL) as a means of removing any particles from the solvent system before the analysis. The HPLC separations were done using a flow rate of $0.5 \mathrm{~mL} / \mathrm{min}$. A Milli- $\mathrm{Q}^{\mathrm{TM}}$ purification system (Millipore Corp., Bedford, MA) was used for the preparation of deionized water.

The other HPLC system used was an 1100 series Agilent instrument equipped with a binary pump, autosampler, degasser, and a UV detector. Chemstation software was used for the data analysis. The pump flow rate used was $0.5 \mathrm{~mL} / \mathrm{min}$ and the column temperature was maintained at ambient. 


\section{RESULTS AND DISCUSSIONS}

\section{A. Spectroscopic Characterization of Bonded Stationary Phases}

\section{Diffuse Reflectance Infrared Fourier Transform Spectra}

\section{a. Silanization}

The DRIFT spectra were used in this research to confirm the $\mathrm{Si}-\mathrm{H}$ bonded stationary phase. The DRIFT spectra of Si-H using Astrosil silica is shown in Figure 8. The very intense absorption band near $2250 \mathrm{~cm}^{-1}$ was assigned to the $\mathrm{Si}-\mathrm{H}$ stretching frequency which confirmed $\mathrm{Si}-\mathrm{H}$ formation after the silanization reaction with TES. The broad band between 3000 and $3700 \mathrm{~cm}^{-1}$ was assigned for the absorbed water and $\mathrm{OH}$ groups from the silica. 


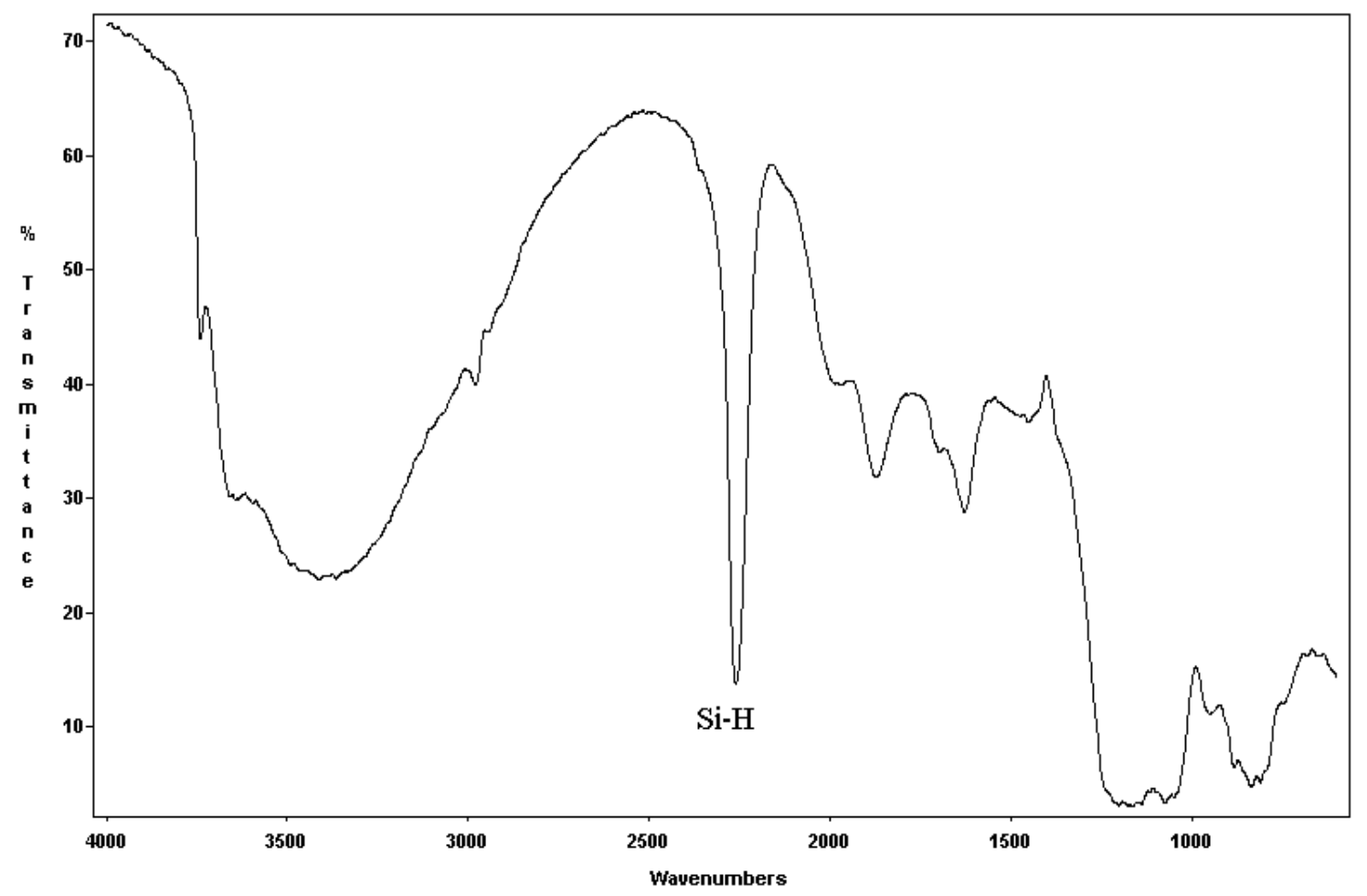

Figure 8. DRIFT spectrum of silica hydride stationary phase.

\section{b. Endcapped Silica Hydride}

Figure 9 shows the DRIFT spectrum of the endcapped silica hydride. The endcapped $\mathrm{Si}-\mathrm{H}$ material gave a lower but significant intensity absorption band near $2250 \mathrm{~cm}^{-1}$, which confirmed the Si-H stretching band. The broad band between 3000 and $3700 \mathrm{~cm}^{-1}$ was assigned for the absorbed water and $\mathrm{OH}$ groups from the silica, this was seen in the silanization reaction step as well. The strong $\mathrm{C}-\mathrm{H}$ stretching vibration band between 2800 to $3000 \mathrm{~cm}^{-1}$ indicated again the formation of an organic bonded phase or the attachement of an oganic group to $\mathrm{Si}-\mathrm{OH}$ surface through the endcapping process. 


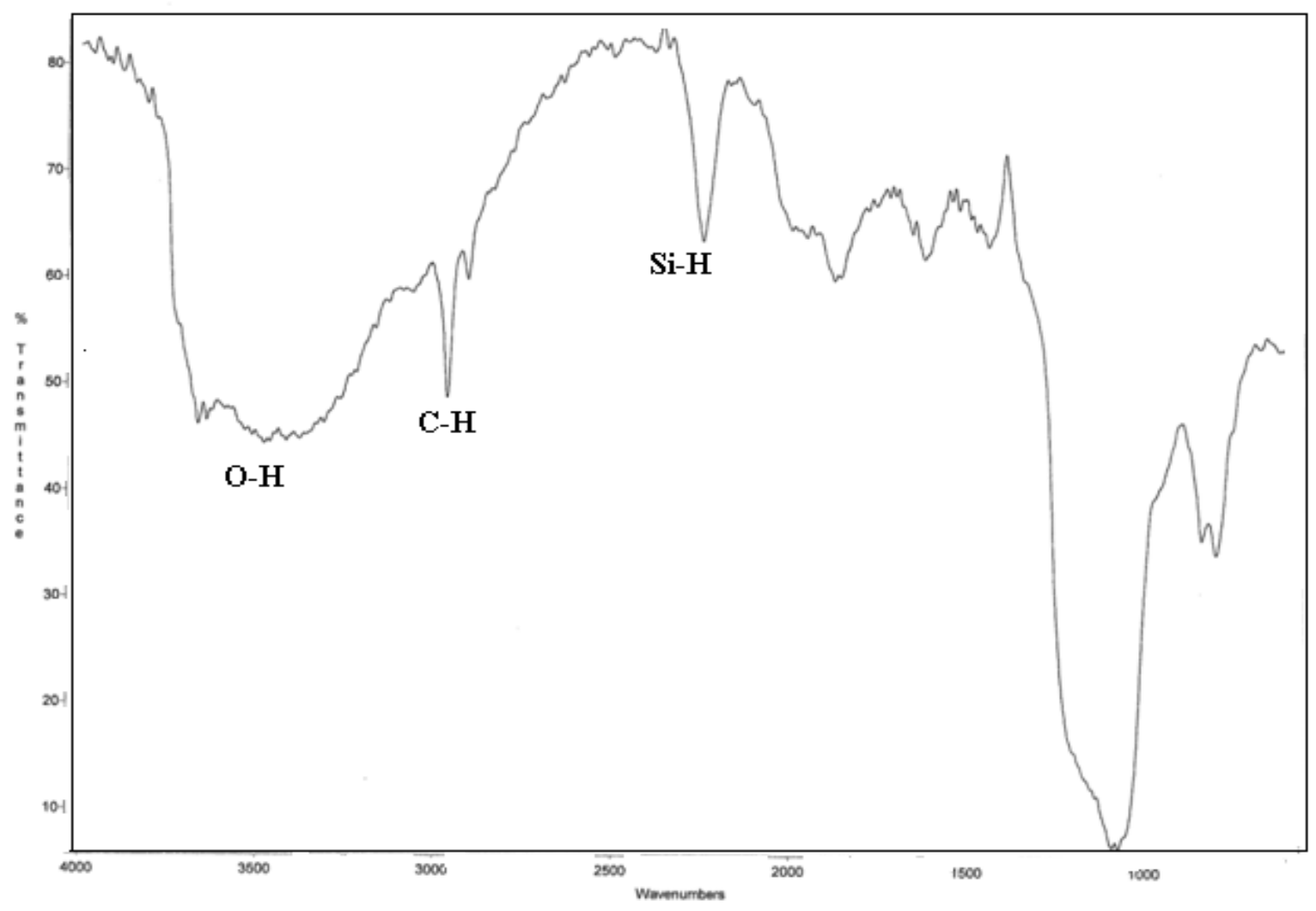

Figure 9. DRIFT spectrum of endcapped Si-H stationary phase.

\section{B. Chromatographic Characterization of Bonded Stationary Phase}

The spectroscopic characterization confirmed the success of bonding after silanization and endcapping process. The Neue test confirmed the hydrophobicity and silanophilicity of the stationary phase. The information obtained was helpful in determining the type of compounds which could be used for chromatographic characterization. Chromatographic characterization helped in determining the potential applications of the new stationary phase material, and the technique used for 
chromatographic characterization was HPLC with either an ELSD detector or UV detector.

\section{Characterization of HPLC Packing by Neue Test}

The hydrophobicity and silanophilicity were determined using four compounds. The information obtained helps to determine the type of compounds that could be retained on the column.

Experimental Conditions: The mobile phase used was 35:65 $20 \mathrm{mM}$ phosphate buffer/methanol, $\mathrm{pH}$ 7. The flow rate used was $0.5 \mathrm{~mL} / \mathrm{min}$ and the injection volume was $1 \mu \mathrm{L}$. The compounds were detected at $214 \mathrm{~nm}$. Two sample mixtures were made. The first mixture contained uracil $(16 \mathrm{mg} / \mathrm{mL})$, naphthalene $(60 \mathrm{mg} / \mathrm{mL})$, and acenaphthene (200 mg/mL) in methanol. The second mixture contained amitryptiline hydrochloride $(100 \mathrm{mg} / \mathrm{mL})$ and acenaphthene $(200 \mathrm{mg} / \mathrm{mL})$ in methanol. Uracil in the first mixture was used as a marker for the column void volume. The solutes used and their retention times at both conditions are shown in Table 5 . 
Table 5. Results of Neue test

\begin{tabular}{|c|c|c|}
\hline Compounds & $\begin{array}{c}\text { Endcapped Si-H Column } \\
(\mathbf{m i n})\end{array}$ & C8 Si-H column (min) \\
\hline Uracil & 1.0 & 4.8 \\
\hline Naphthalene & 2.2 & 6.0 \\
\hline Acenaphthene & 2.3 & 1.7 \\
\hline Amitryptiline hydrochloride & 2.1 & \\
\hline
\end{tabular}




\section{a. Hydrophobicity Result of Endcapped Si-H Stationary Phase}

The mixture of uracil, naphthalene, and acenaphthene in methanol was run on the endcapped column to measure the hydrophobicity of the stationary phase.

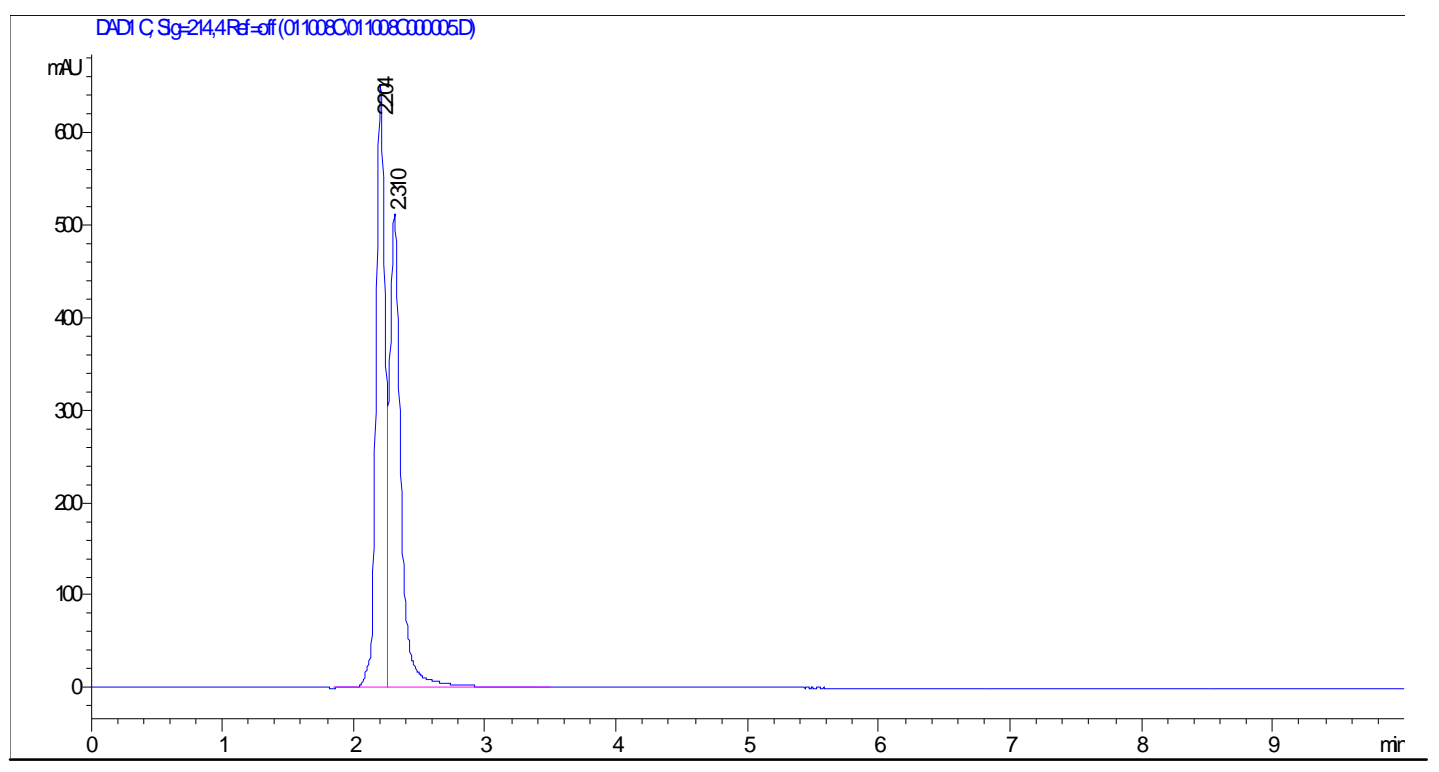

Figure 10. Hydrophobicity test on endcapped silica hydride phase. $2.204 \mathrm{~min}$ is naphthalene and $2.310 \mathrm{~min}$ is acenaphthene.

Naphthalene eluted at a retention time $2.204 \mathrm{~min}$, acenaphthene at $2.31 \mathrm{~min}$, and uracil eluted at the solvent front which did not show up for the endcapped silica hydride stationary phase as shown in Figure 10. The hydrophobicity parameter was calculated for the endcapped column as shown below:

\section{Hydrophobicity Parameter(u)}

$$
\ln (\mathrm{k})=\mathrm{u} * \mathrm{~V}-3.068
$$

$\mathrm{V}=$ Molar volume of the analyte (acenaphthene or naphthalene), which is $200 \mathrm{mM}$ 


$$
\mathrm{k}=\left(\mathrm{t}_{\mathrm{r}}-\mathrm{t}_{0}\right) / \mathrm{t}_{0}
$$

$t_{r}=2.31 \mathrm{~min}$, the retention time of acenaphthene and $t_{0}=1.0 \mathrm{~min}$, the retention time of the void volume that was obtained separately by running uracil.

$$
\mathrm{k}=(2.31-1.0) / 1.0=1.31
$$

Hydrophobicity of endcapped Si-H: $\ln (1.31$, Acenaphthene $)+3.068 / 200=0.016$ The hydrophobicity of endcapped $\mathrm{Si}-\mathrm{H}$ column was 0.02 in comparison to $\mathrm{C} 18$ silica hydride column which is 0.04 [19] as shown in Table 6.

\section{b. Silanophilicity Result of Endcapped Si-H Stationary Phase}

The mixture of amitryptiline and acenaphthene dissolved in methanol was run on the endcapped column to measure the silanophilicity of the stationary phase.

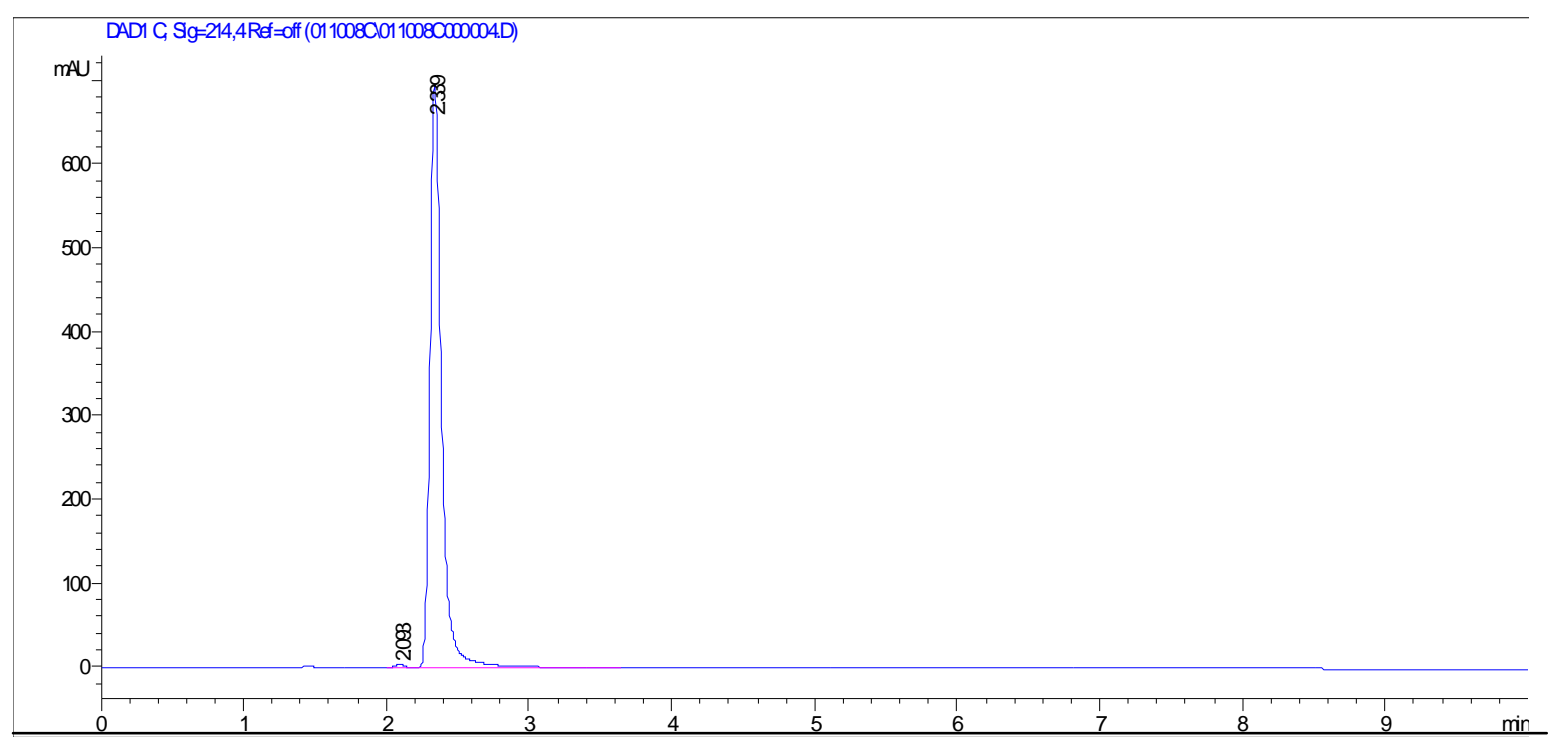

Figure 11. Silanophobicity test on endcapped silica hydride. $2.093 \mathrm{~min}$ is amitryptiline and $2.310 \mathrm{~min}$ is acenaphthene. 
Amitryptiline eluted at a retention time $2.093 \mathrm{~min}$ and acenaphthene at $2.310 \mathrm{~min}$ as shown in Figure 11. The silanophilicity was calculated for the endcapped column as shown below:

$$
\begin{gathered}
\mathrm{S}=\ln (\text { kamitryptiline })-0.7124 * \ln (\text { kacenaphthene })+1.9748 \\
\mathrm{k}=\left(\mathrm{t}_{\mathrm{r}}-\mathrm{t}_{0}\right) / \mathrm{t}_{0}
\end{gathered}
$$

$\mathrm{t}_{\mathrm{r}}=2.093 \mathrm{~min}$, the retention time of amitryptiline and $\mathrm{t}_{0}=1.0 \mathrm{~min}$, the retention time of the void volume that was obtained separately by running uracil.

$$
\mathrm{k}=(2.093-1.0) / 1.0=1.09
$$

$\mathrm{k}=1.09$ for amitryptiline

$\mathrm{t}_{\mathrm{r}}=2.310 \mathrm{~min}$, the retention time of acenaphthene

$\mathrm{k}=1.31$ for acenaphthene

$$
\ln (1.09, \text { Amitryptiline })-0.7124 * \ln (1.31, \text { Acenaphthene })+1.9748=1.80
$$

The silanophilicity of endcapped $\mathrm{Si}-\mathrm{H}$ column was 1.80 in comparison to $\mathrm{C} 18$ silica hydride column which is 1.43 [19] as shown in Table 6.

\section{c. Hydrophobicity Result of C8 Stationary Phase}

The mixture of uracil, naphthalene, and acenaphthene in methanol was run on the C8 column to measure the hydrophobicity of the stationary phase. 


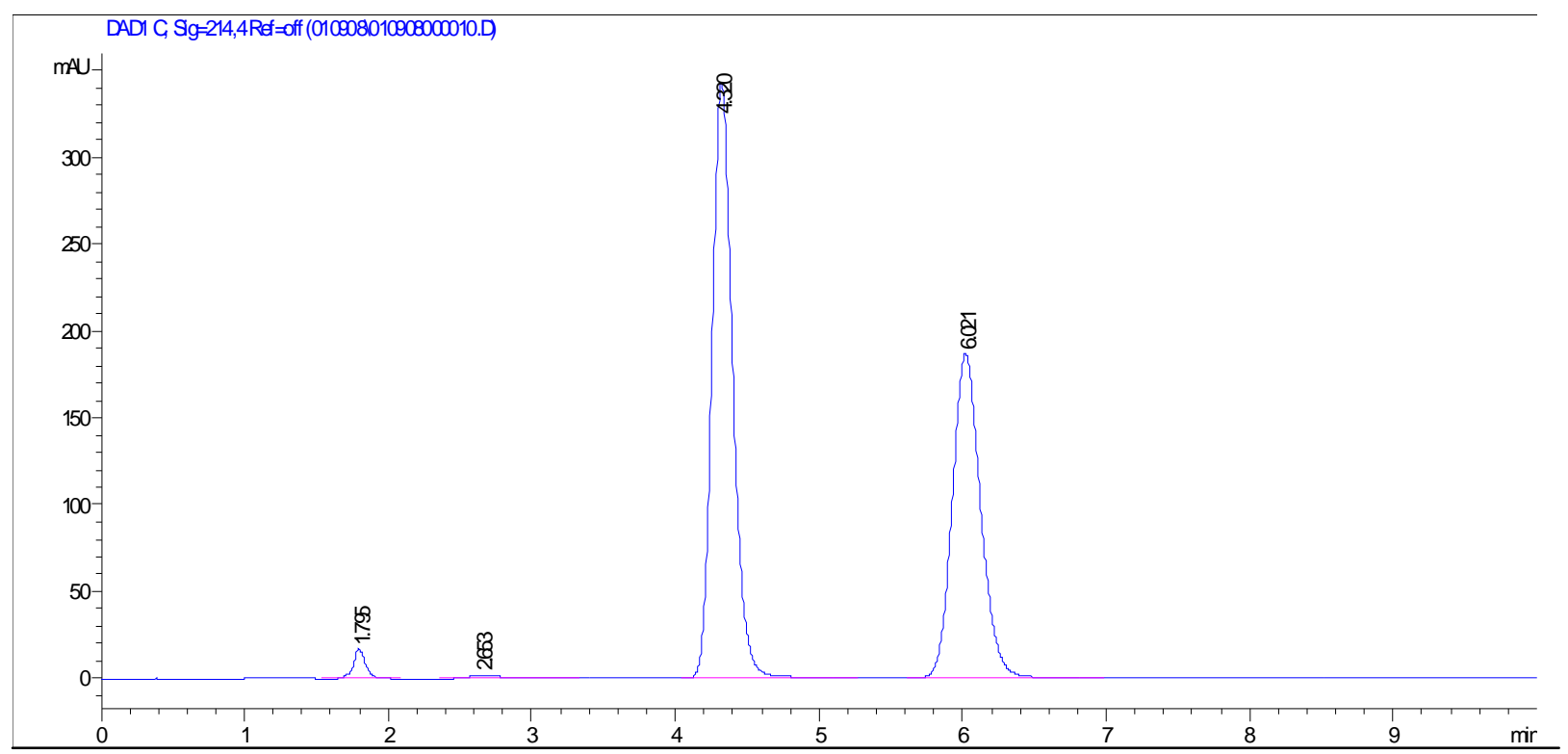

Figure 12. Hydrophobicity test on C8 phase. $1.795 \mathrm{~min}$ uracil, $4.320 \mathrm{~min}$ naphthalene, and $6.021 \mathrm{~min}$ acenaphthene.

Naphthalene eluted at a retention time $4.320 \mathrm{~min}$, acenaphthene at $6.021 \mathrm{~min}$, and uracil eluted at $1.795 \mathrm{~min}$ at the solvent front as shown in Figure 12. The calculation of the hydrophobicity parameter for the C8 column:

\section{Hydrophobicity Parameter(u)}

$$
\ln (\mathrm{k})=\mathrm{u} * \mathrm{~V}-3.068
$$

$\mathrm{V}=$ Molar volume of the analyte (acenaphthene or naphthalene), which is $200 \mathrm{mM}$

$$
\mathrm{k}=\left(\mathrm{t}_{\mathrm{r}}-\mathrm{t}_{0}\right) / \mathrm{t}_{0}
$$

$\mathrm{t}_{\mathrm{r}}=6.02 \mathrm{~min}$, the retention time of acenaphthene and $\mathrm{t}_{0}=1.795 \mathrm{~min}$, the retention time of the void volume.

$$
\mathrm{k}=(6.02-1.795) / 1.795=2.35
$$

Hydrophobicity of C8 column: 
$\ln (2.35$, Acenaphthene $)+3.068 / 200=0.02$

The hydrophobicity of C8 Si-H column was 0.02 in comparison to $\mathrm{C} 18$ silica hydride column which is 0.04 [19] as shown in Table 6.

\section{d. Silanophilicity Result of C8 Stationary Phase}

The mixture of amitryptiline and acenaphthene in methanol was run on the C8 column to measure the silanophilicity of the stationary phase.

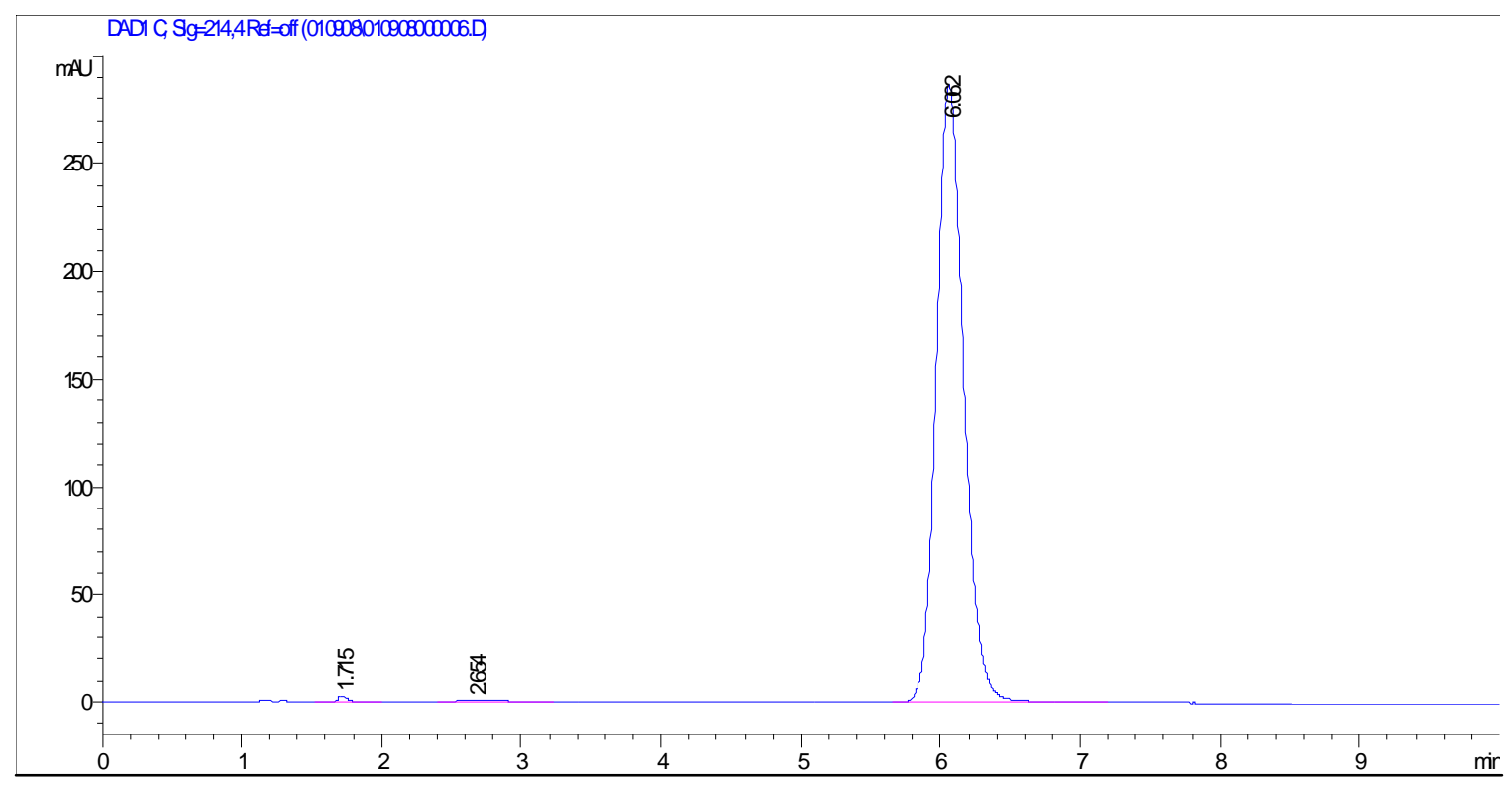

Figure 13. Silanophobicity test on C8 column. 2.654 min amitryptiline and 6.062 min acenaphthene.

Amitryptiline eluted at a retention time $2.654 \mathrm{~min}$ and acenaphthene at $6.062 \mathrm{~min}$ as shown in Figure 13. The calculation of the silanophilicity factor for the C8 column:

$$
\begin{gathered}
\mathrm{S}=\ln (\text { kamitryptiline })-0.7124 * \ln (\text { kacenaphthene })+1.9748 \\
\mathrm{k}=\left(\mathrm{t}_{\mathrm{r}}-\mathrm{t}_{0}\right) / \mathrm{t}_{0}
\end{gathered}
$$


$\mathrm{t}_{\mathrm{r}}=2.654 \mathrm{~min}$, the retention time of amitryptiline and $\mathrm{t}_{0}=1.795 \mathrm{~min}$, the retention time for the void volume.

$$
\mathrm{k}=(2.654-1.795) / 1.795=0.478
$$

$\mathrm{k}=0.478$ for amitryptiline

$t_{\mathrm{r}}=6.062 \mathrm{~min}$, the retention time of acenaphthene

$\mathrm{k}=2.377$ for acenaphthene

$\ln (1.715 \mathrm{~min}$, Amitryptiline $)-0.7124 * \ln (6.06 \mathrm{~min}$, Acenaphthene $)+1.9748=0.399$

The silanophilicity of C8 Si-H column was 0.4 in comparison to $\mathrm{C} 18$ silica hydride column which is 1.43 [19] as shown in Table 6.

Table 6. Summary of hydrophobicity and silanophilicity results of C8 and endcapped silica hydride stationary phases

\begin{tabular}{|c|c|c|c|c|}
\hline & & & \multicolumn{2}{|c|}{ From article [19] } \\
\hline Columns & Hydrophobicity & Silanophilicity & Hydrophobicity & Silanophilicity \\
\hline C18 & NA & NA & 0.0397 & 1.429 \\
\hline C8 & 0.02 & 0.399 & - & - \\
\hline Endcapped Si-H & 0.016 & 1.8 & - & - \\
\hline
\end{tabular}

- = The information is not available.

The comparison of hydrophobicity and silanophilicity data between $\mathrm{C} 18, \mathrm{C} 8$, and endcapped $\mathrm{Si}-\mathrm{H}$ is shown in Table 6. The relative degree of hydrophobicity between the 
columns was as expected. The $\mathrm{C} 18$ column was higher than $\mathrm{C} 8$ column, which was greater than the endcapped Si-H column. This correlates well with the length of the side chain attached to the surface; a longer aliphatic chain results in higher hydrophobicity. The comparison of silanophilicity data between the three columns does not show any trend; however a lower value results in less surface adsorption. Therefore the C8 column has less adsorption when compared to the C18 and the endcapped silica hydride columns.

\section{ANP and RP Behavior of Endcapped Si-H Using an ELSD Detector}

\section{a. Aqueous Normal Phase Behavior of Citric Acid}

Amino acids, vitamins, weak organic acids, chelators, and lipoic acid were used for the chromatographic characterization of the endcapped silica hydride column and the results were compared with the C8 (75 x $4.6 \mathrm{~mm}$ i.d) silica hydride column. The endcapped column was packed in a 75 x $4.6 \mathrm{~mm}$ i.d. stainless steel tube, which was purchased from Alltech, Deerfield, IL.

The chromatographic characterization of the columns was done using HPLC with UV and an ELSD detector. The parameters used for HPLC with the ELSD detector were a flow rate of $0.5 \mathrm{~mL} / \mathrm{min}$, an injection volume of $5 \mu \mathrm{L}$, and the column temperature at ambient. The concentration of the samples was $0.5 \mathrm{mg} / \mathrm{mL}$ in a 50:50 water/acetonitrile solution. The ELSD parameters were set and stabilized with the starting conditions of the isocratic system. The temperature of the drift tube and spray chamber were adjusted until a smooth baseline was obtained. The spray chamber was kept at room temperature 
$\left(25^{\circ} \mathrm{C}\right)$ for all the analyses and the drift tube temperature was set at $65^{\circ} \mathrm{C}$ to evaporate the mobile phase. The composition of the mobile phase used was $0.1 \%$ formic acid (FA) because it was compatible with the ELSD detector and was very easy to evaporate during light scattering detection. A water blank was run to eliminate any carryover from the previous measurements. The main goal of this part of the research was to evaluate the endcapped stationary phase for aqueous normal phase and reversed-phase behavior.

The concentration of citric acid used for aqueous normal phase behavior was $0.5 \mathrm{mg} / \mathrm{mL}$ in a 50:50 water/acetonitrile solution. The ELSD detector parameters were set and stabilized with the starting conditions of the isocratic system.

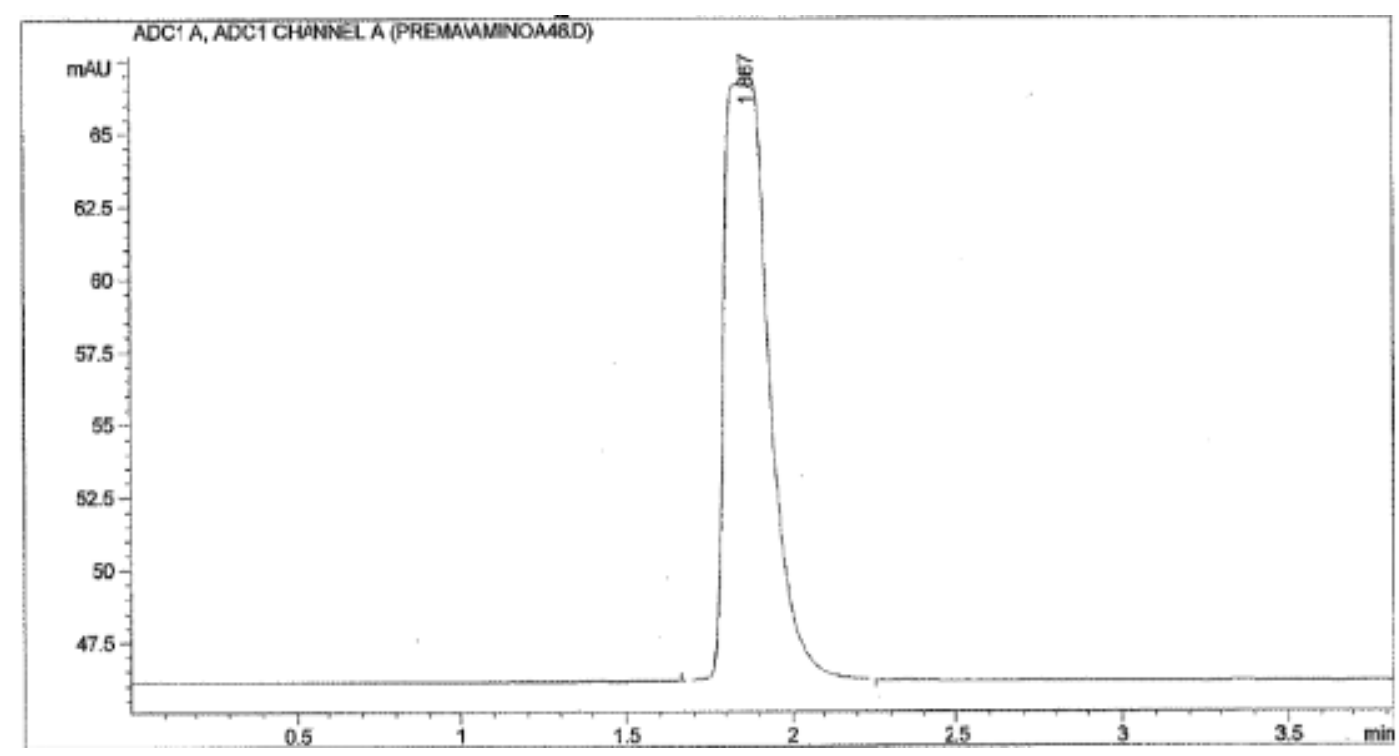

Figure 14. Chromatogram of citric acid on endcapped silica hydride stationary phase. Mobile phase: acetonitrile $+0.1 \%$ formic acid. 


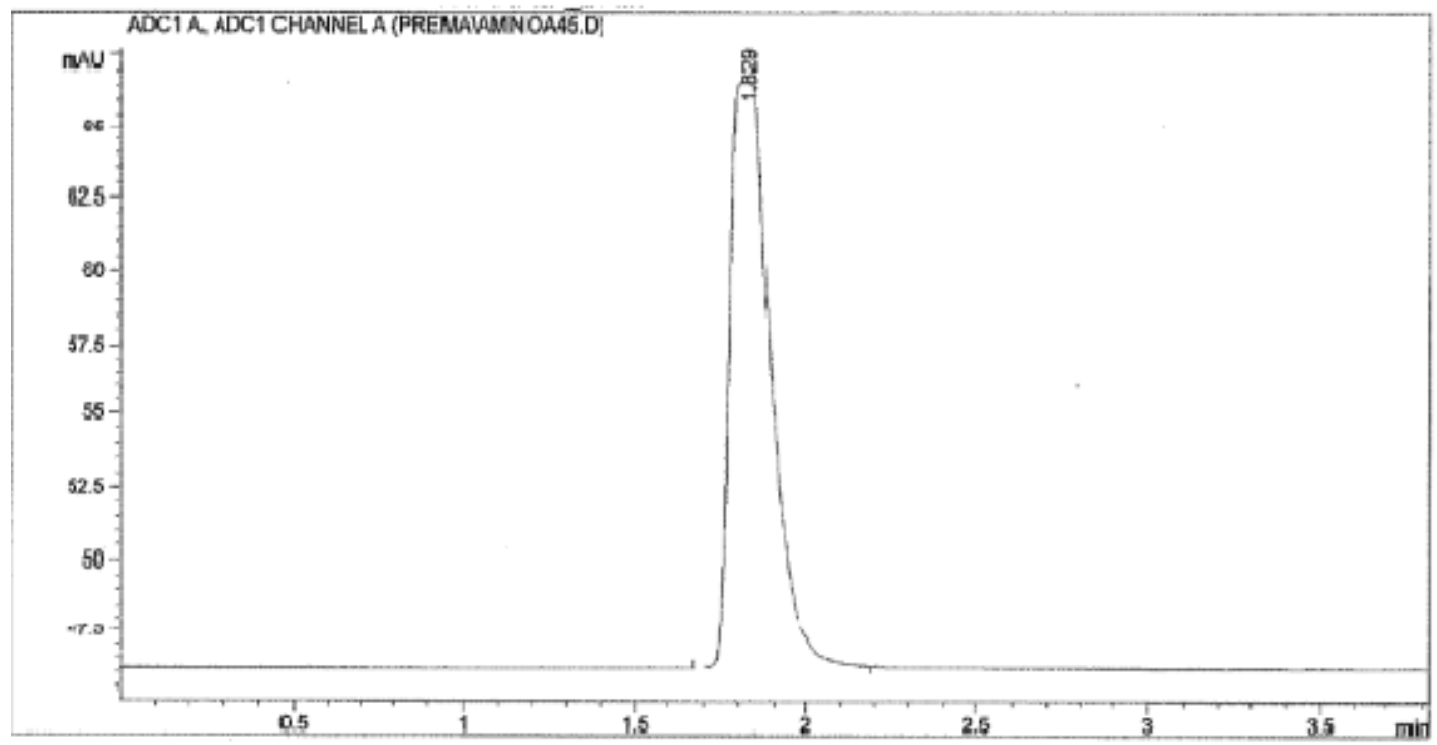

Figure 15. Chromatogram of citric acid on endcapped silica hydride stationary phase. Mobile phase: $10: 90$ water $+0.1 \%$ formic acid / acetonitrile $+0.1 \%$ formic acid. 


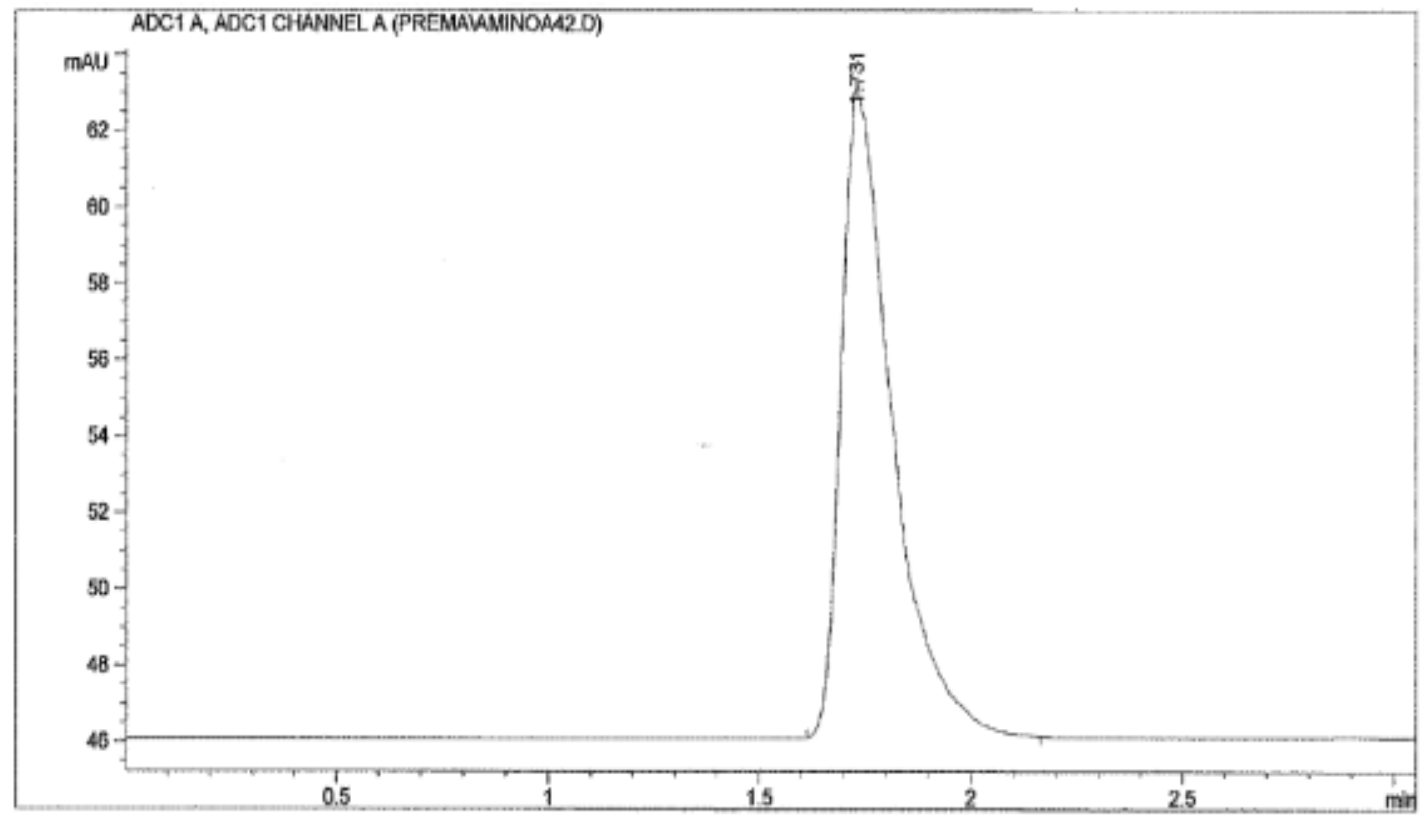

Figure 16. Chromatogram of citric acid on endcapped silica hydride stationary phase. Mobile phase: $20: 80$ water $+0.1 \%$ formic acid / acetonitrile $+0.1 \%$ formic acid. 


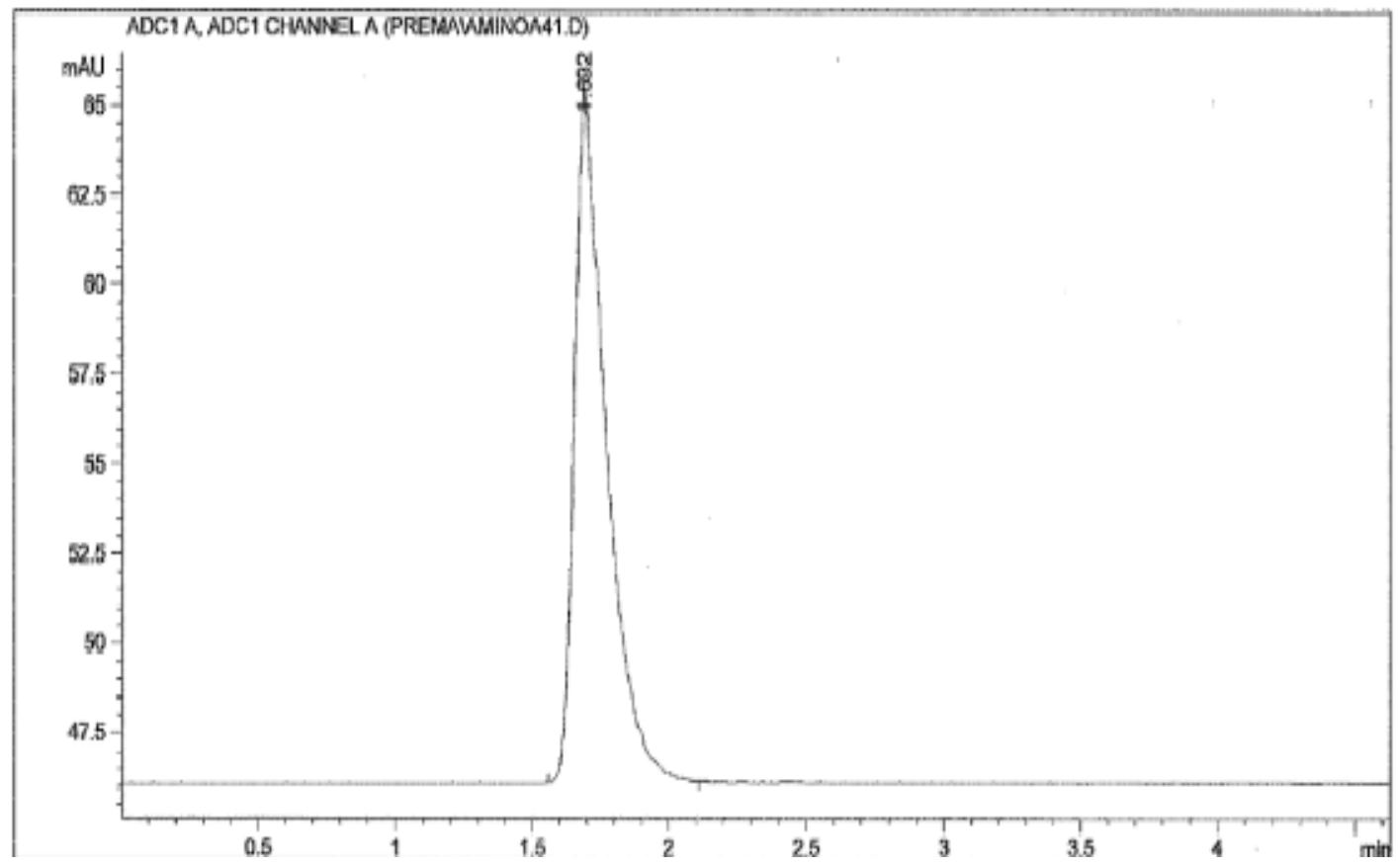

Figure 17. Chromatogram of citric acid on endcapped silica hydride stationary phase. Mobile phase: $30: 70$ water $+0.1 \%$ formic acid / acetonitrile $+\mathbf{0 . 1 \%}$ formic acid. 


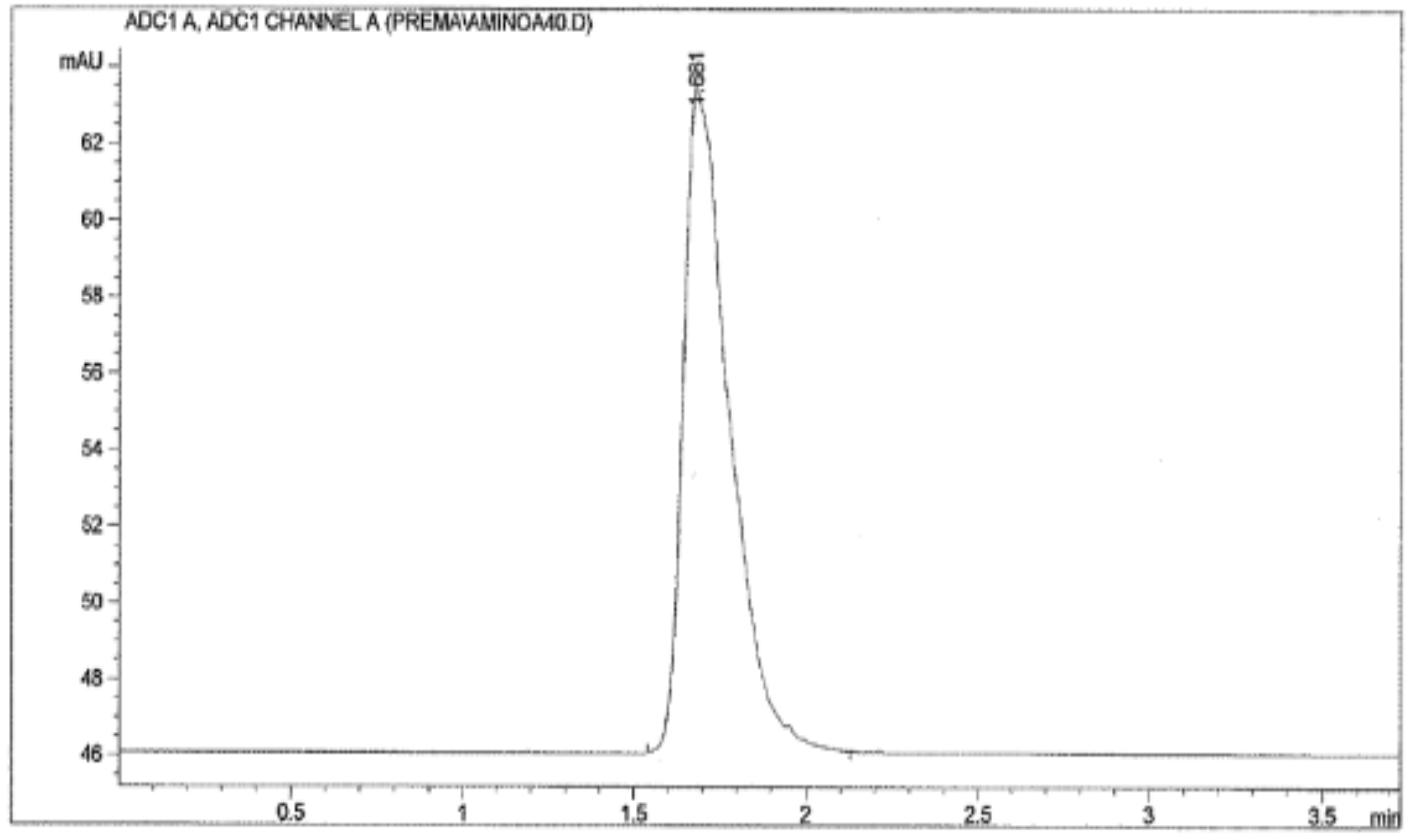

Figure 18. Chromatogram of citric acid on endcapped silica hydride stationary phase. Mobile phase: 40:60 water $+0.1 \%$ formic acid / acetonitrile $+0.1 \%$ formic acid. 


\section{b. Reversed-Phase Behavior of Citric Acid}

The concentration of citric acid used for reversed-phase behavior was $0.5 \mathrm{mg} / \mathrm{mL}$ in a 50:50 water/acetonitrile solution. The ELSD detector parameters were set and stabilized with the starting conditions of the isocratic system.

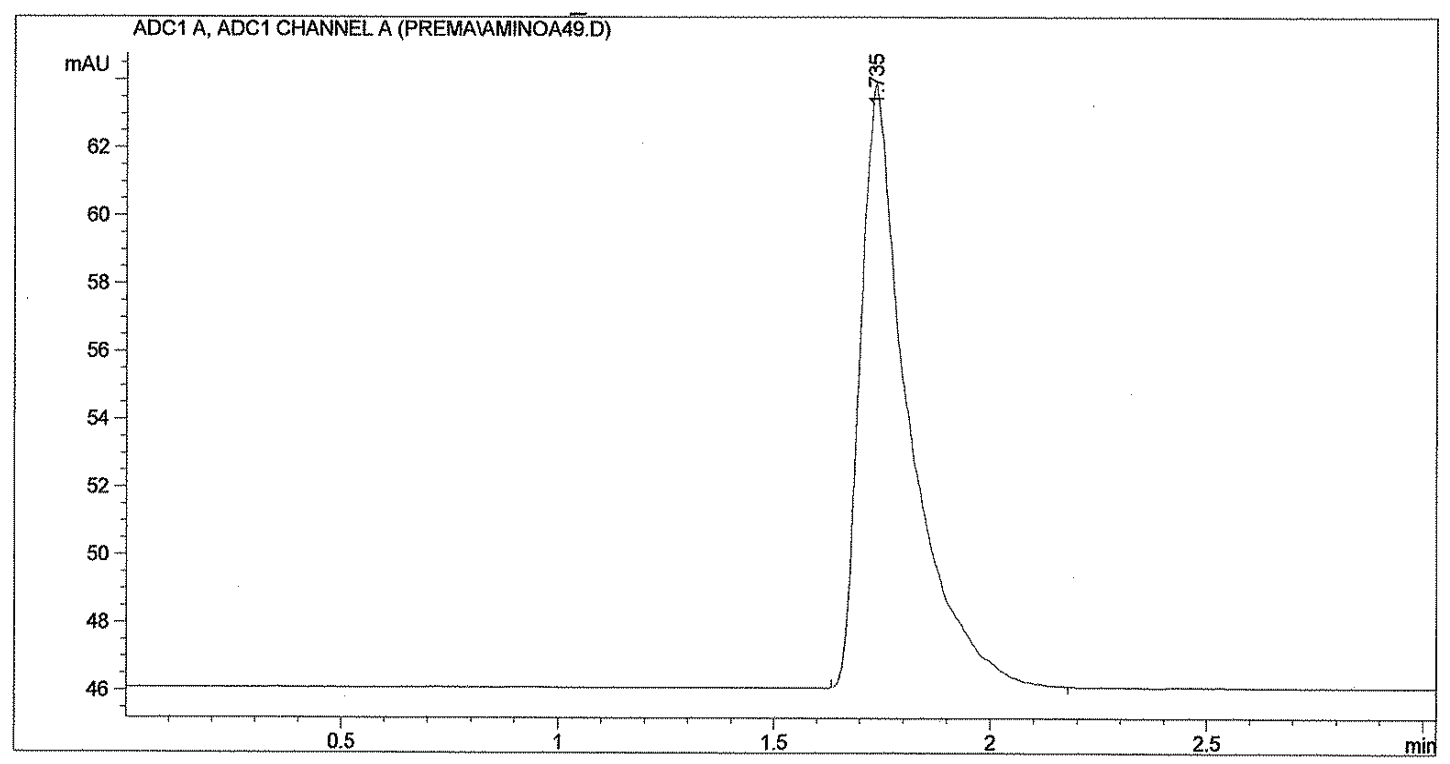

Figure 19. Chromatogram of citric acid on endcapped silica hydride stationary phase. Mobile phase: $60: 40$ water $+0.1 \%$ formic acid / acetonitrile $+0.1 \%$ formic acid. 


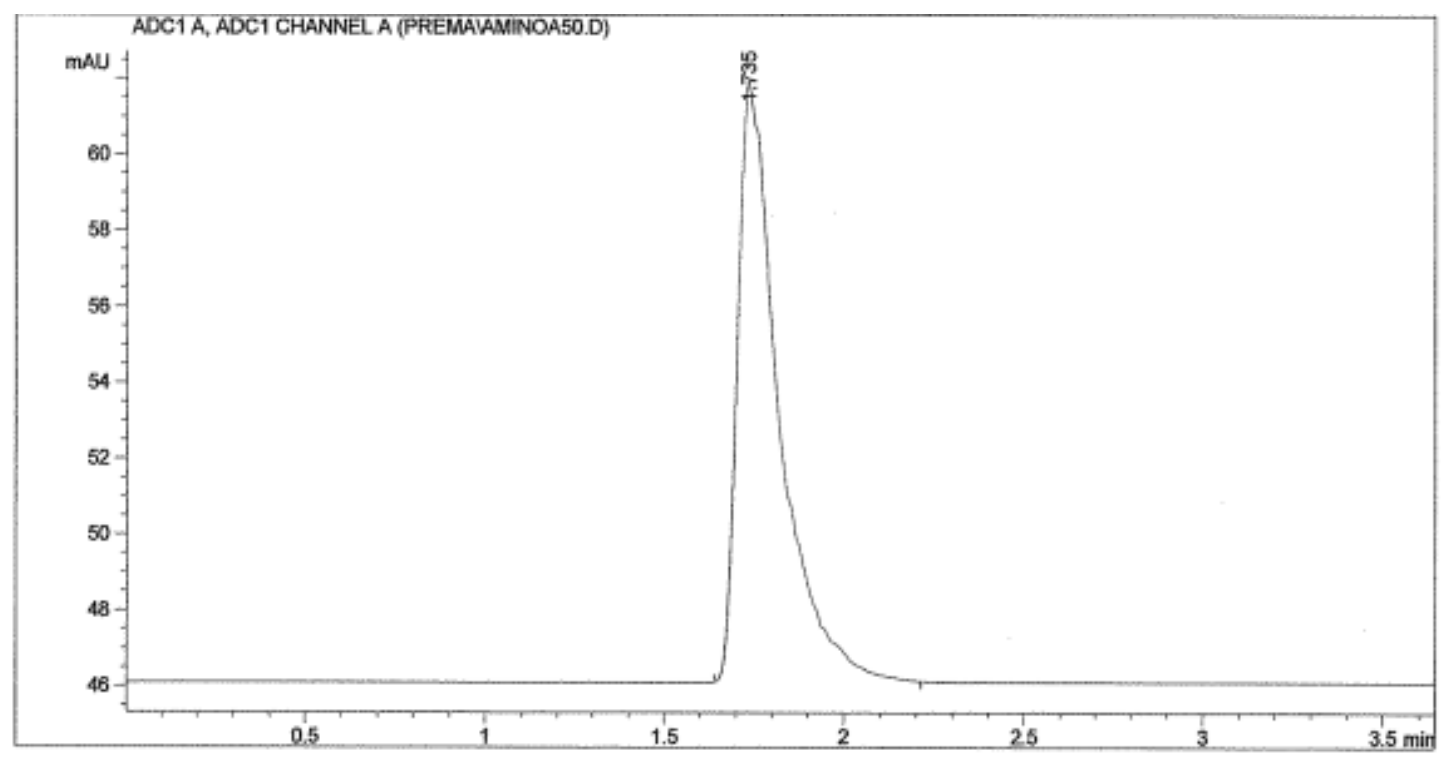

Figure 20. Chromatogram of citric acid on endcapped silica hydride stationary phase. Mobile phase: 70:30 water $+0.1 \%$ formic acid / acetonitrile $+0.1 \%$ formic acid. 


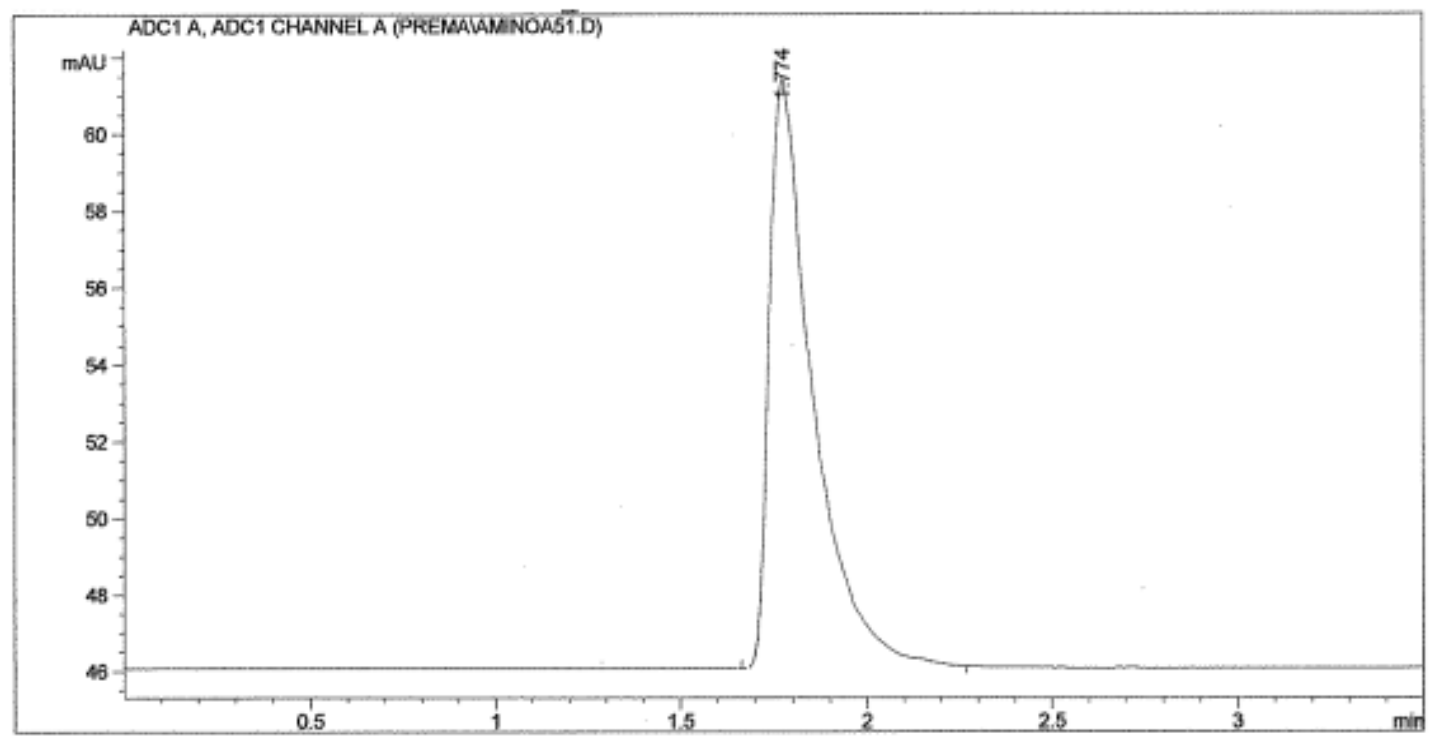

Figure 21. Chromatogram of citric acid on endcapped silica hydride stationary phase. Mobile phase: 80:20 water $+0.1 \%$ formic acid / acetonitrile $+0.1 \%$ formic acid. 


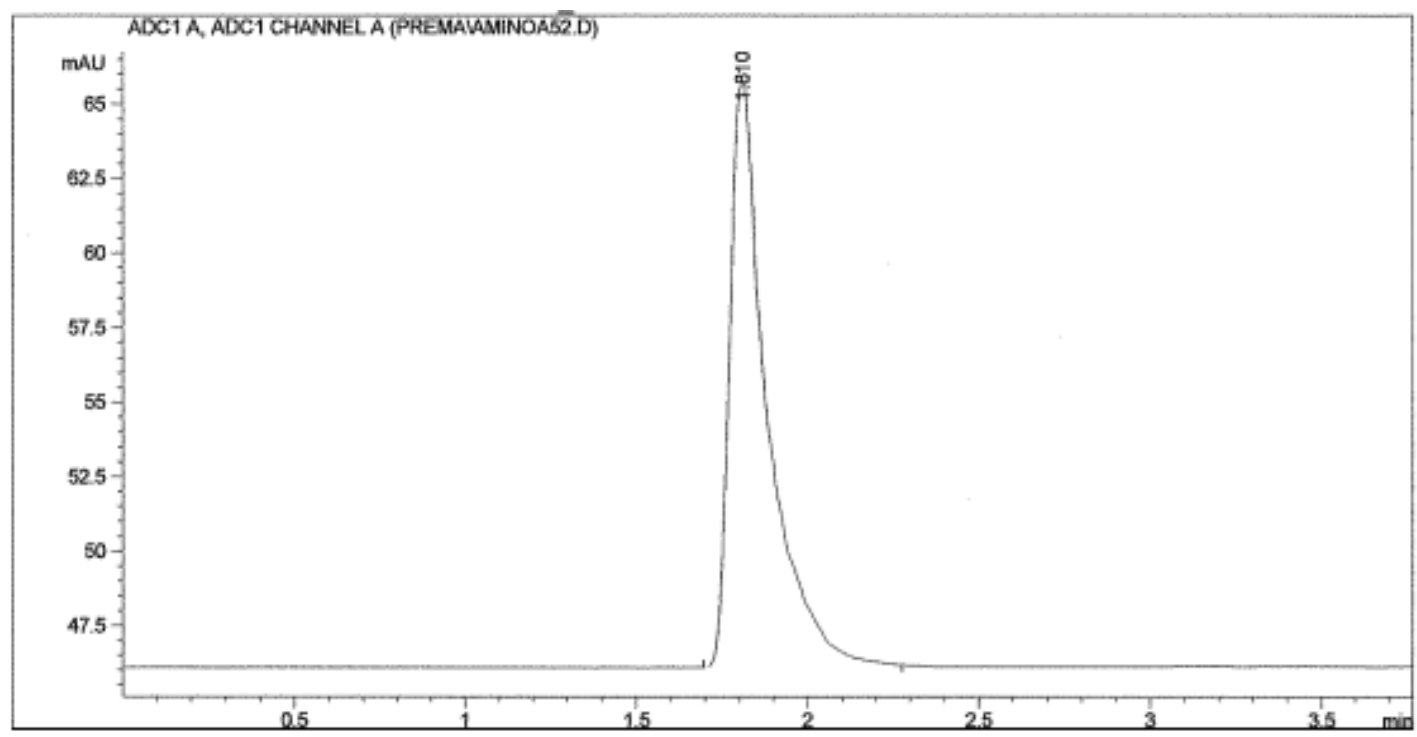

Figure 22. Chromatogram of citric acid on endcapped silica hydride stationary phase. Mobile phase: $90: 10$ water $+0.1 \%$ formic acid / acetonitrile $+0.1 \%$ formic acid. 


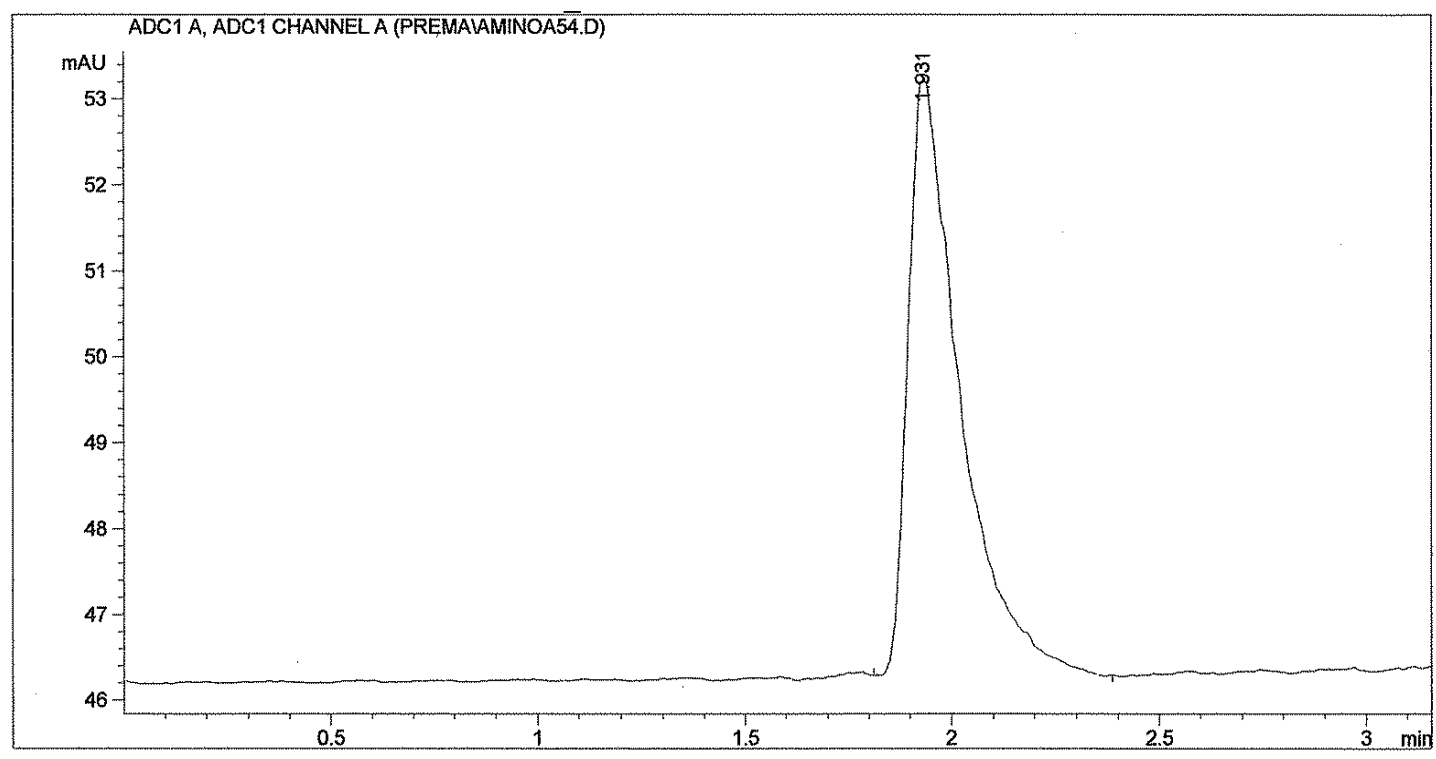

Figure 23. Chromatogram of citric acid on endcapped silica hydride stationary phase. Mobile phase: acetonitrile $+\mathbf{0 . 1 \%}$ formic acid. 


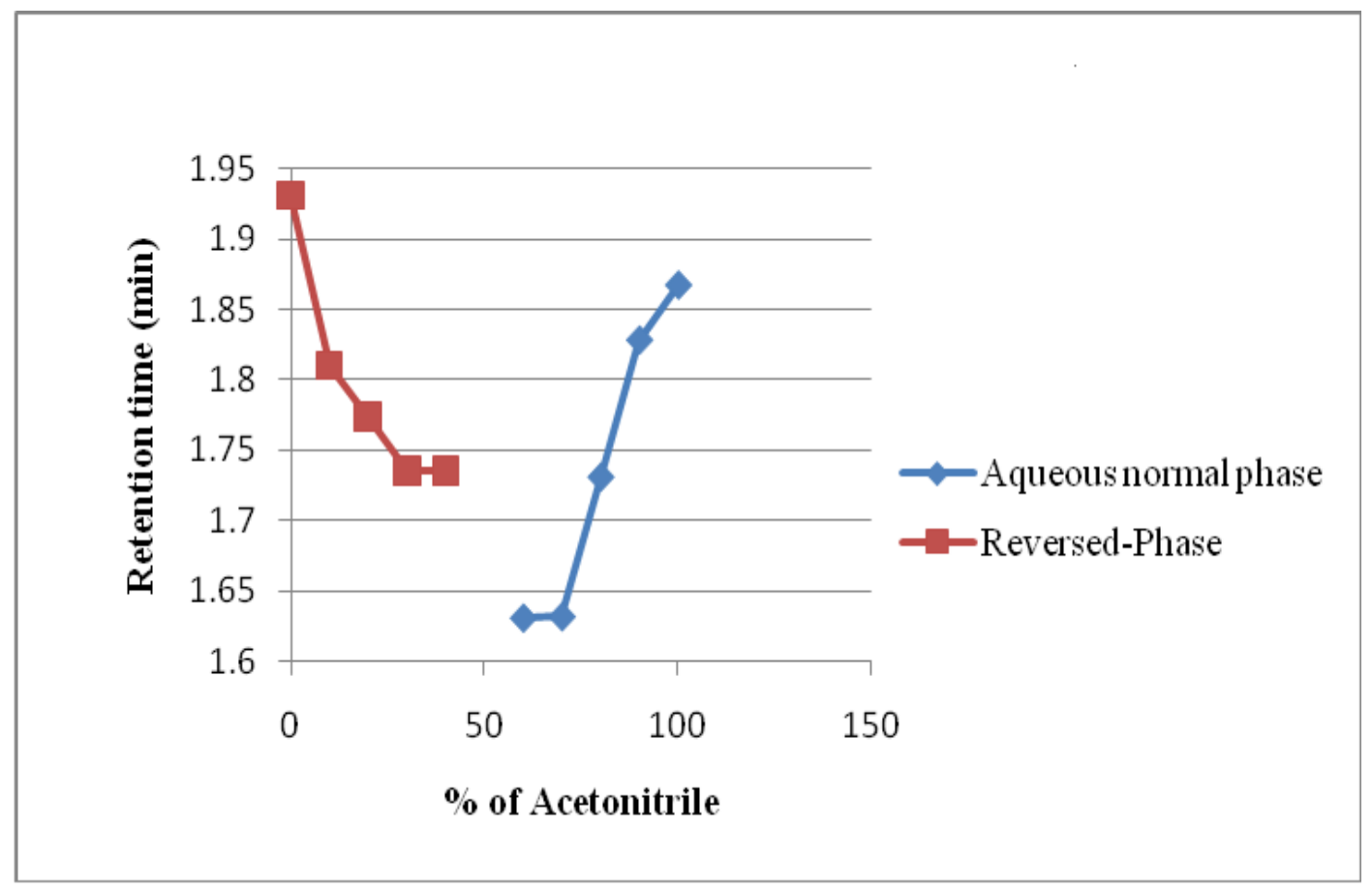

Figure 24. Retention map of citric acid for ANP and RP conditions.

Citric acid was analyzed using aqueous normal phase and reversed-phase conditions as shown in the chromatograms from Figure 14 to Figure 22. The mobile phase used was $0.1 \%$ formic acid in acetonitrile and $0.1 \%$ formic acid in water and, the detector used for the analysis was the evaporative light scattering detector. As shown in Figure 24, citric acid can be retained using the aqueous normal phase elution mode and the reversed-phase elution mode. In the aqueous normal phase mode, the retention of the compound increased as the percentage of acetonitrile increased. It can be retained using the reversed-phase elution mode also as it displayed good retention under selected reversed-phase isocratic conditions. Citric acid was also tested with $0.1 \%$ ammonium 
acetate in water and $0.1 \%$ ammonium acetate in acetonitrile using a UV detector. It was also measured using $0.1 \%$ formic acid in water and $0.1 \%$ formic acid in acetonitrile with a UV detector. The chromatograms for all the isocratic conditions tested had many split peaks and some conditions had negative peaks. Therefore more investigations need to be done using different mobile phase compositions.

\section{c. Reversed-Phase Behavior of Melamine}

The concentration of melamine used for reversed-phase behavior was $0.5 \mathrm{mg} / \mathrm{mL}$ in a 50:50 water/acetonitrile solution. The ELSD detector parameters were set and stabilized with the starting conditions of the isocratic system.

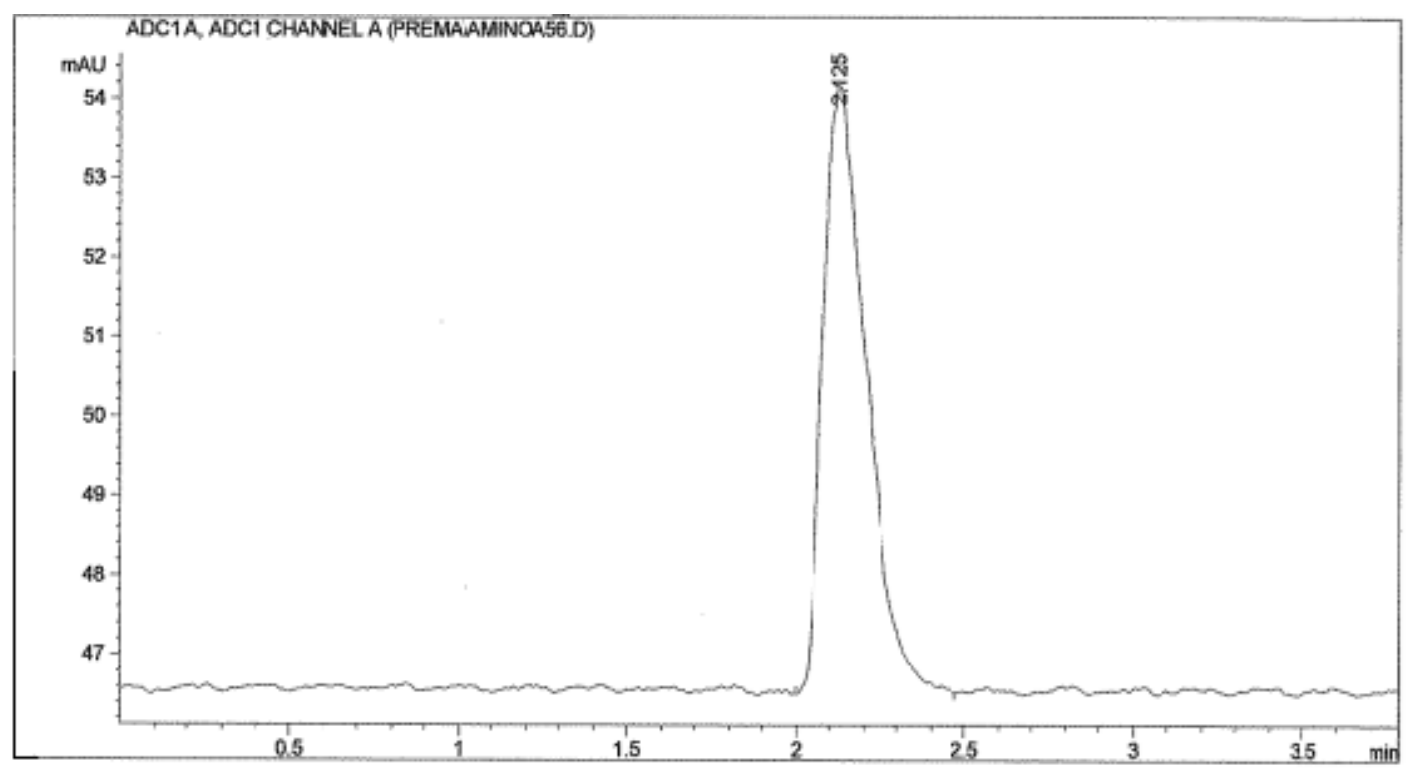

Figure 25. Chromatogram of melamine on endcapped silica hydride stationary phase. Mobile phase: $90: 10$ water $+0.1 \%$ formic acid / acetonitrile $+0.1 \%$ formic acid. 


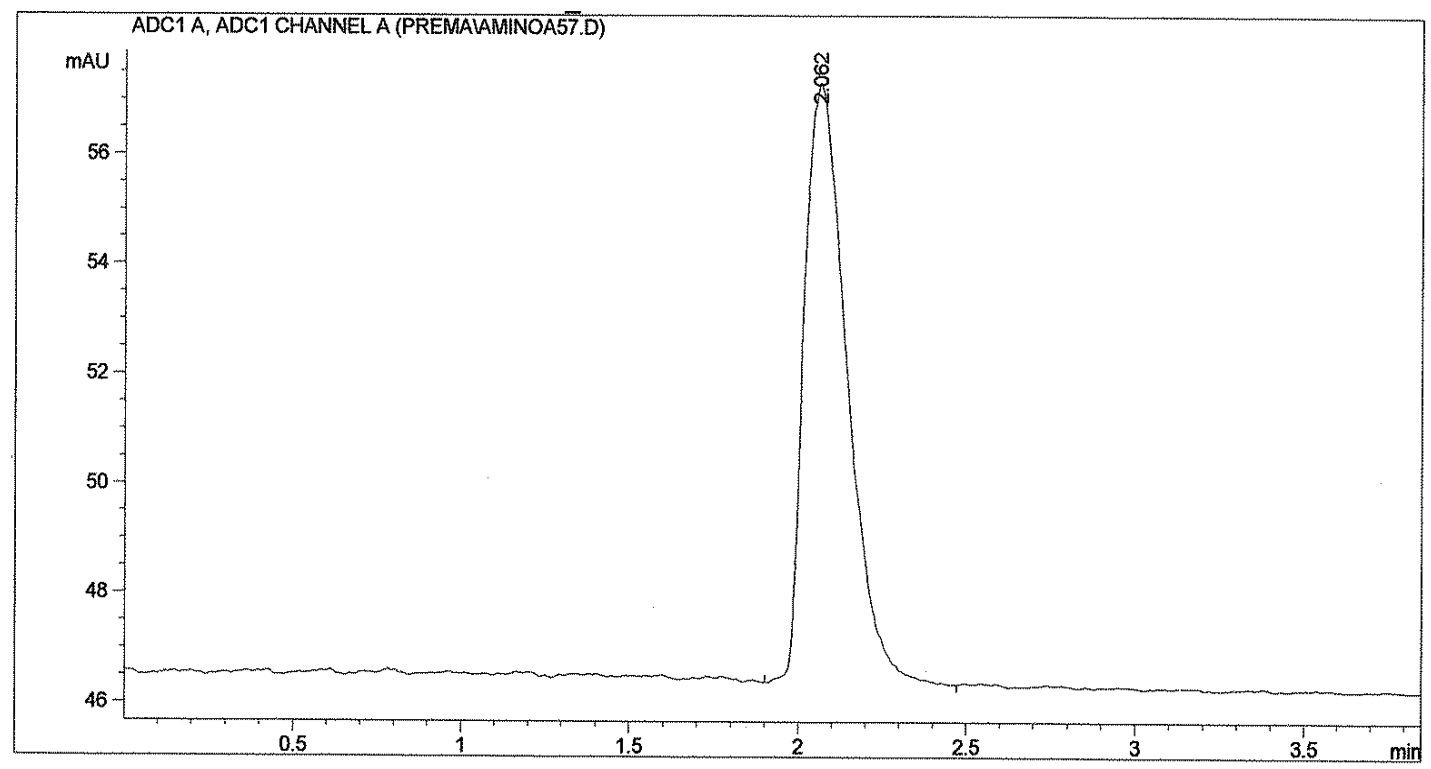

Figure 26. Chromatogram of melamine on endcapped silica hydride stationary phase. Mobile phase: $80: 20$ water $+0.1 \%$ formic acid / acetonitrile $+0.1 \%$ formic acid. 


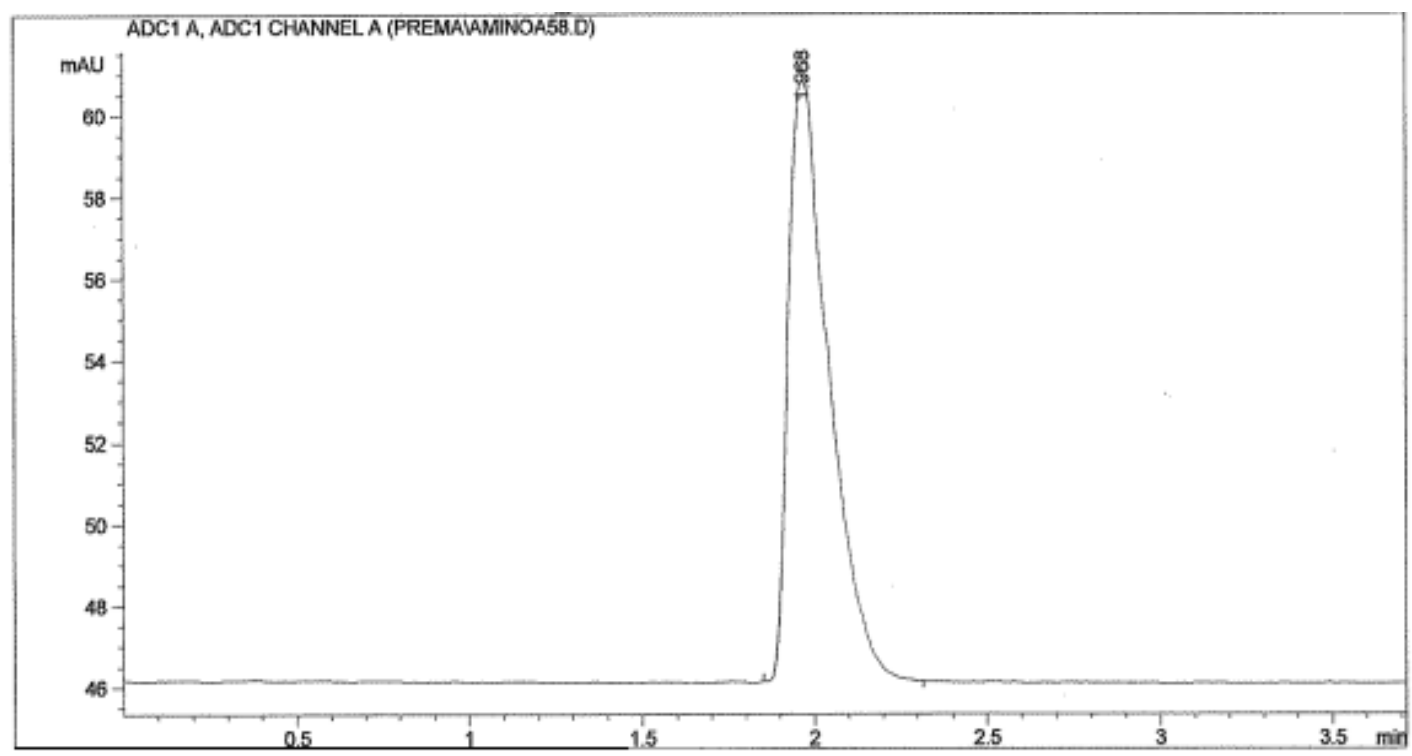

Figure 27. Chromatogram of melamine on endcapped silica hydride stationary phase. Mobile phase: $70: 30$ water $+0.1 \%$ formic acid / acetonitrile $+0.1 \%$ formic acid. 


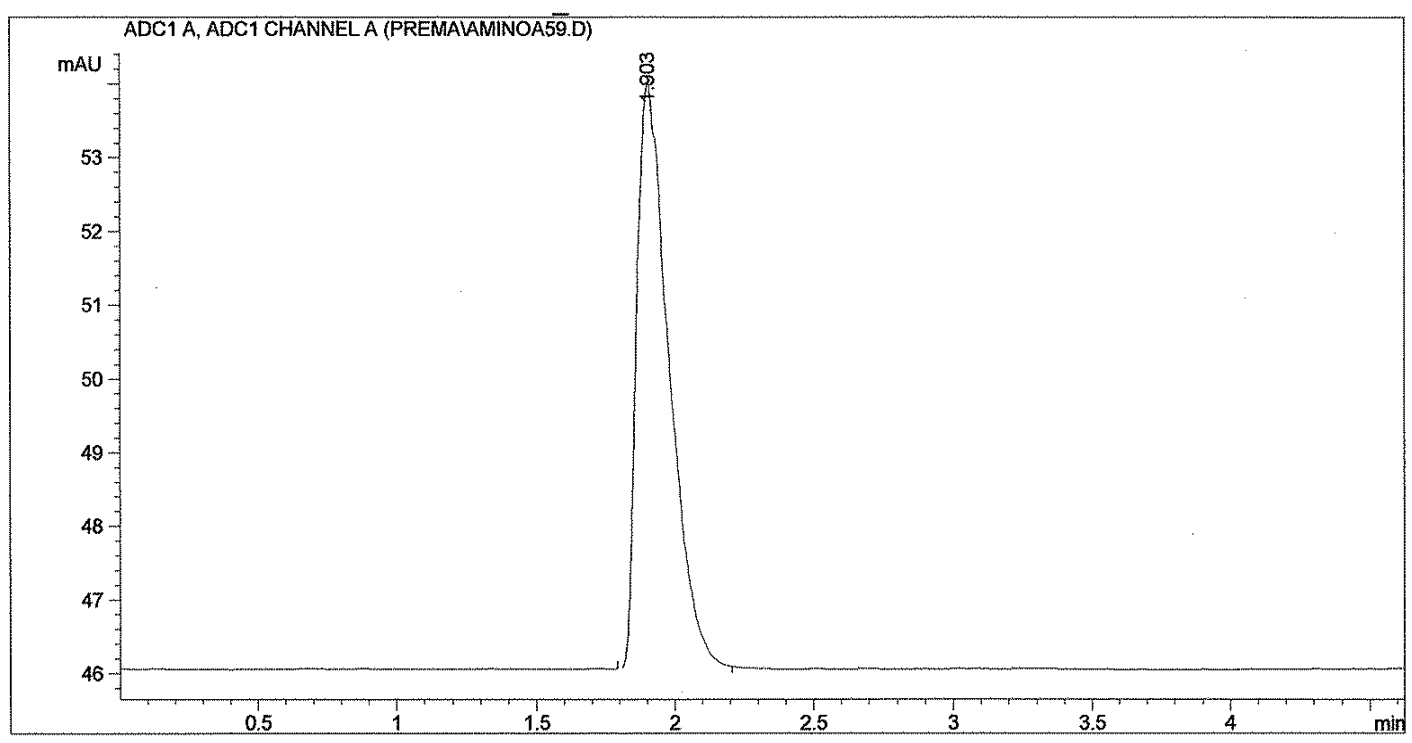

Figure 28. Chromatogram of melamine on endcapped silica hydride stationary phase. Mobile phase: $60: 40$ water $+0.1 \%$ formic acid / acetonitrile $+0.1 \%$ formic acid. 


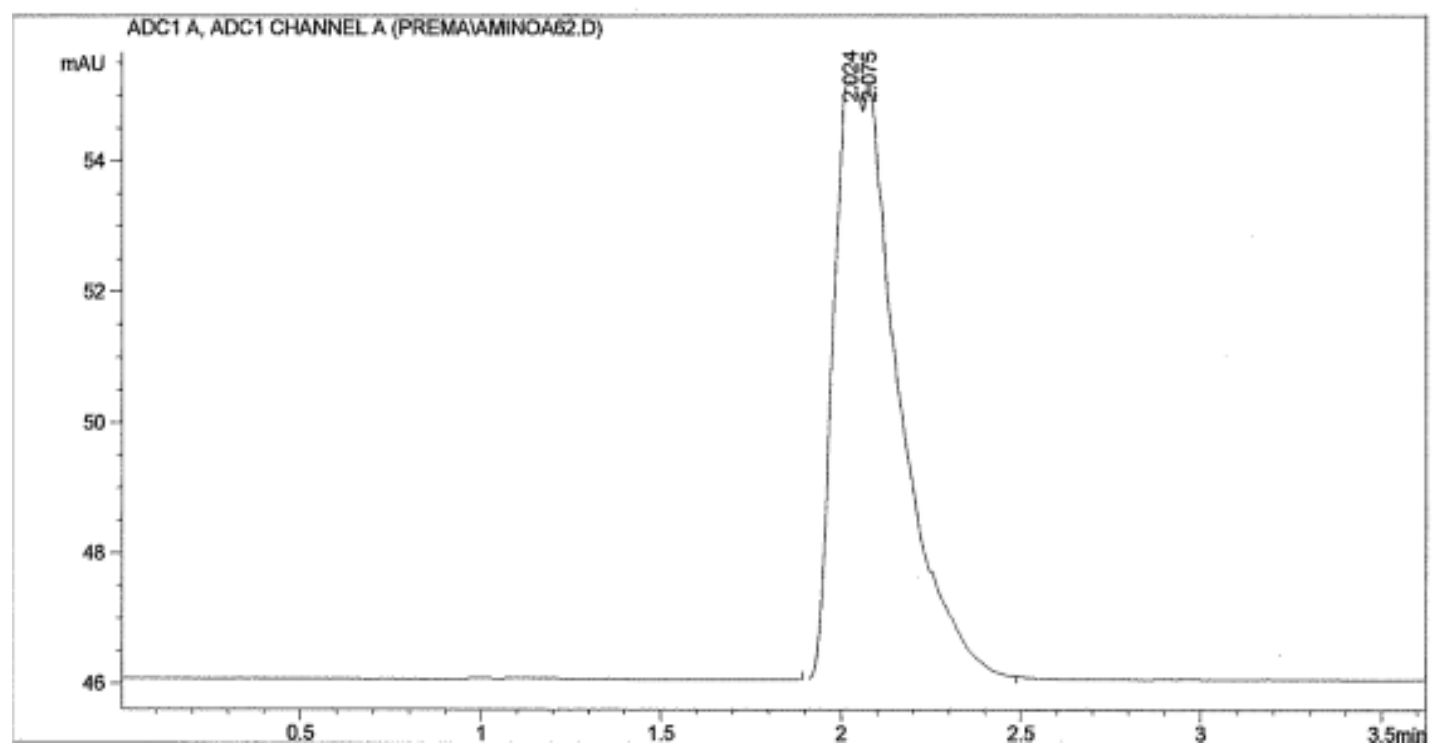

Figure 29. Chromatogram of melamine on endcapped silica hydride stationary phase. Mobile phase: $50: 50$ water $+0.1 \%$ formic acid / acetonitrile $+0.1 \%$ formic acid. 


\section{d. Aqueous Normal Phase Behavior of Melamine}

The concentration of melamine used for aqueous normal phase behavior was $0.5 \mathrm{mg} / \mathrm{mL}$ in a 50:50 water/acetonitrile solution. The ELSD detector parameters were set and stabilized with the starting conditions of the isocratic system.

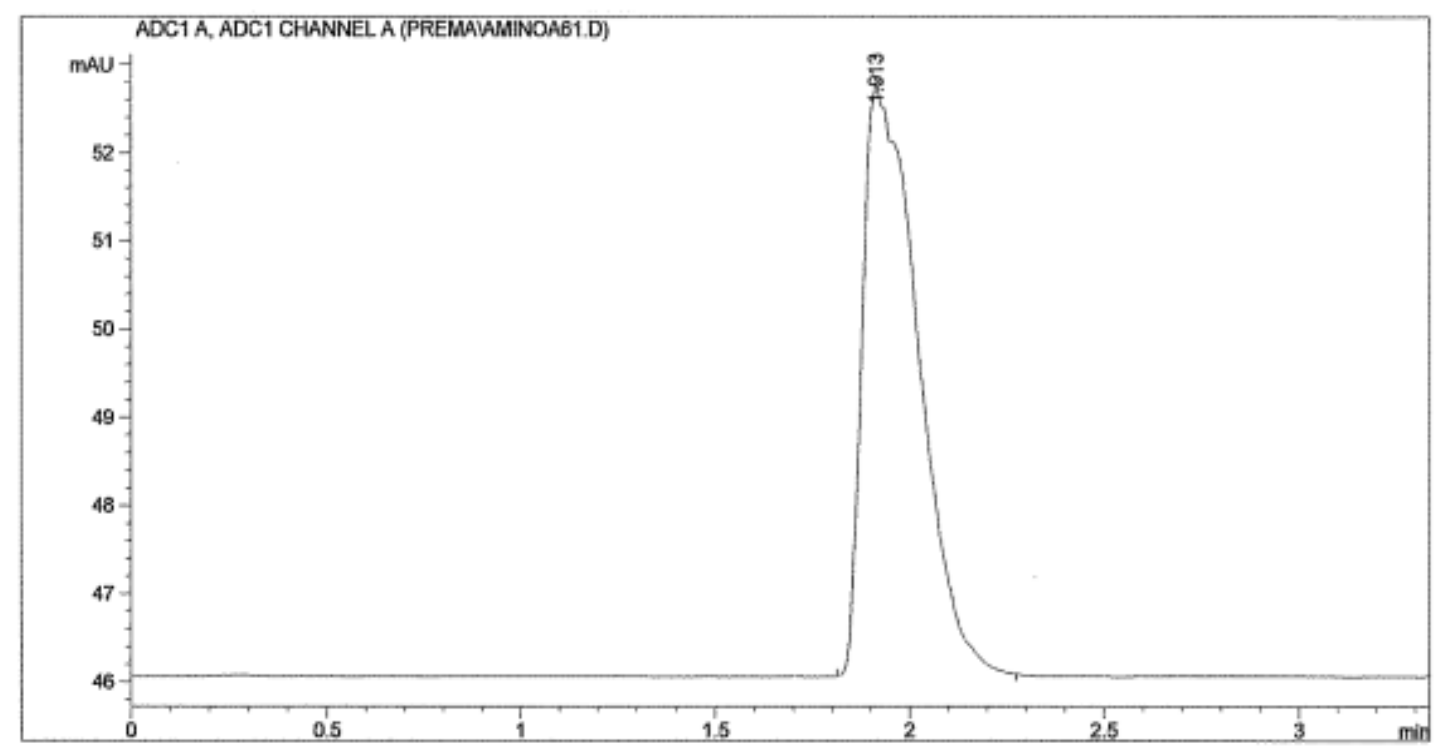

Figure 30. Chromatogram of melamine on endcapped silica hydride stationary

phase. Mobile phase: 40:60 water $+0.1 \%$ formic acid / acetonitrile $+0.1 \%$ formic acid. 


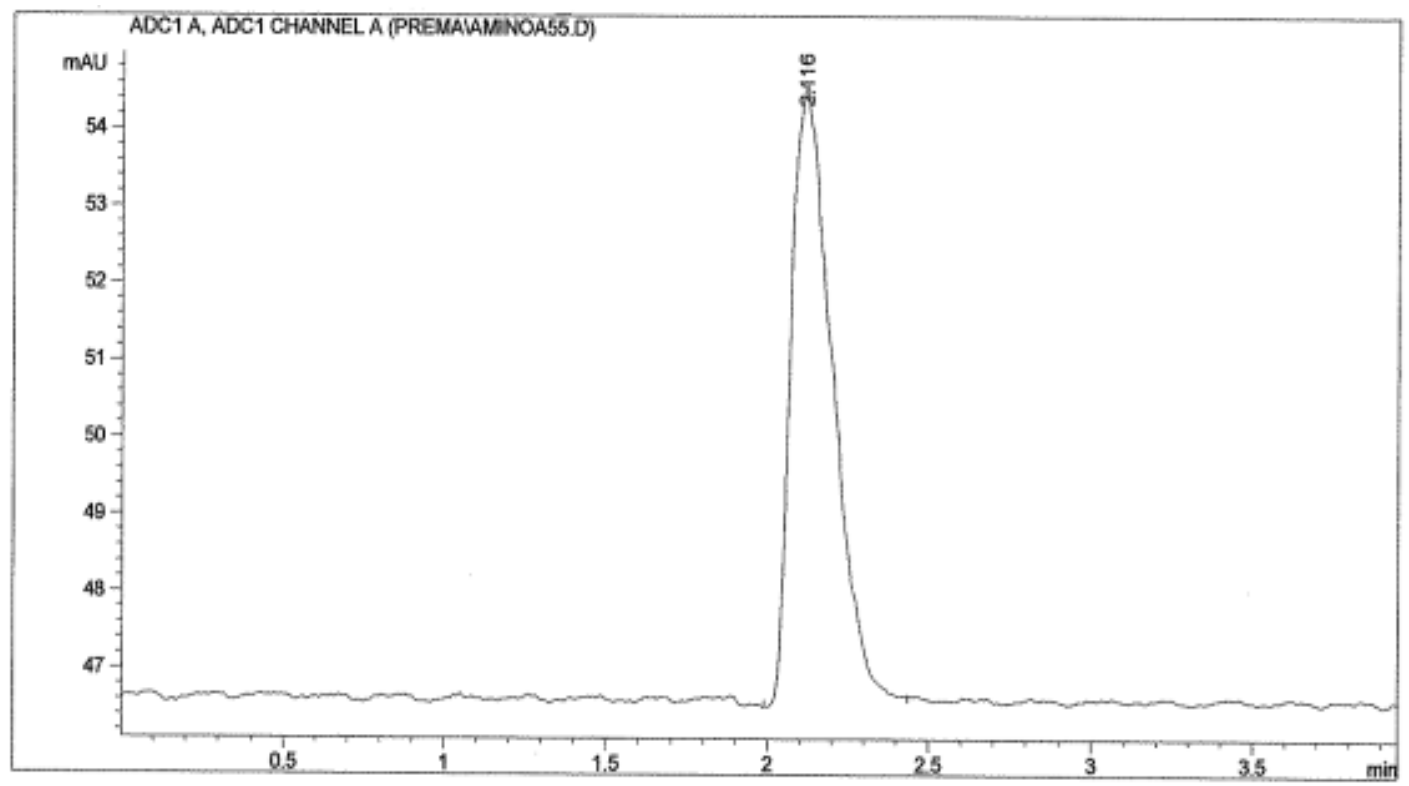

Figure 31. Chromatogram of melamine on endcapped silica hydride stationary phase. Mobile phase: $20: 80$ water $+0.1 \%$ formic acid / acetonitrile $+0.1 \%$ formic acid. 


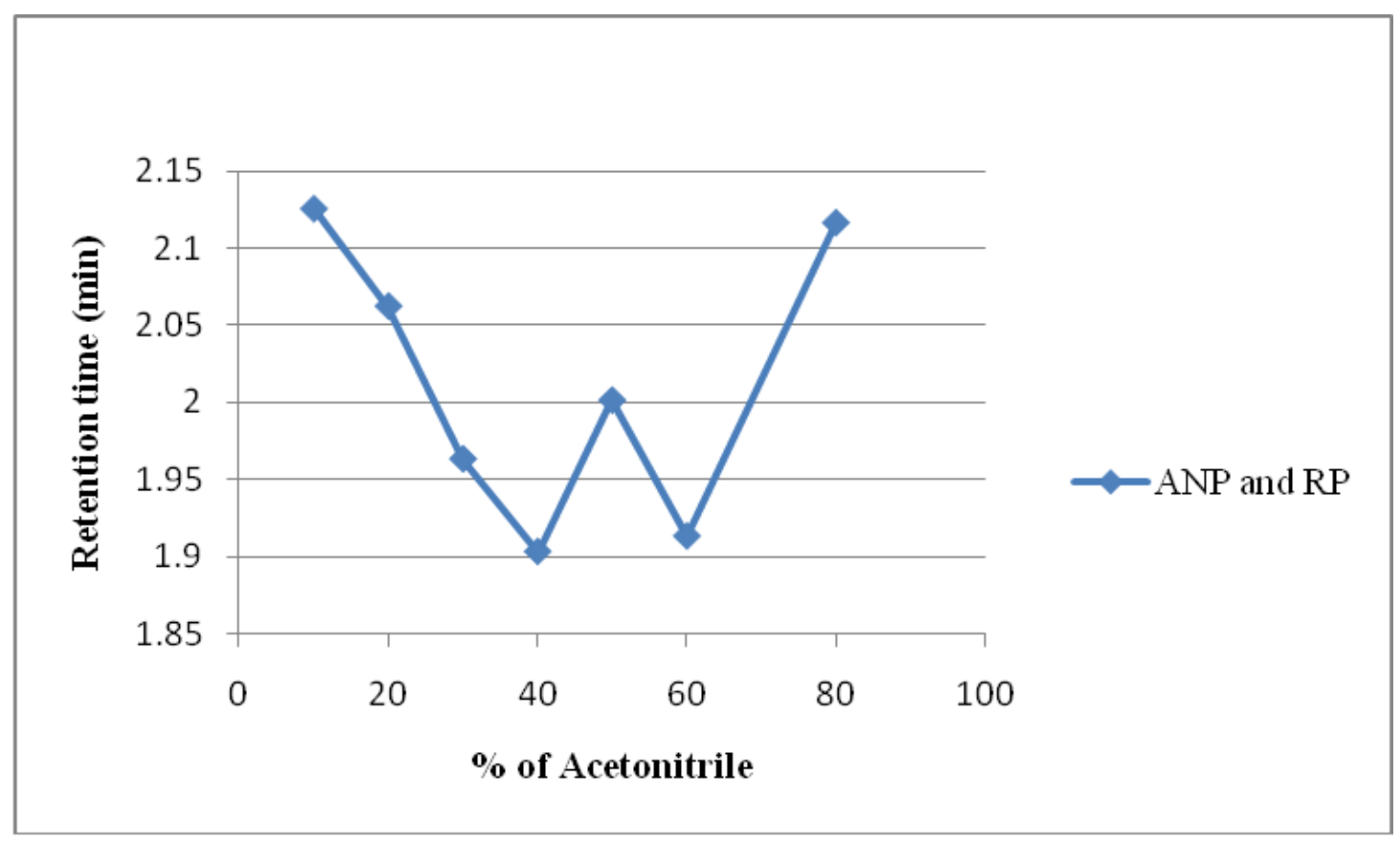

Figure 32. Retention map of melamine for ANP and RP conditions.

Melamine was analyzed using aqueous normal phase as well as using reversed-phase conditions as shown in the chromatograms from Figure 25 to Figure 31. The mobile phase used was $0.1 \%$ formic acid in acetonitrile and $0.1 \%$ formic acid in water. The detector used for the analysis was the evaporative light scattering detector. As shown in Figure 32, melamine can be retained using the aqueous normal phase elution mode. The basic principle of the aqueous normal phase mode is that retention increases as the percentage of acetonitrile increases. Melamine can also be retained in the reversed-phase elution mode as shown in Figure 32 for the isocratic conditions with high water content. 


\section{e. Aqueous Normal Phase Behavior of Phenylalanine}

The concentration of phenylalanine used for aqueous normal phase behavior was $0.5 \mathrm{mg} / \mathrm{mL}$ in a 50:50 water/acetonitrile solution. The ELSD detector parameters were set and stabilized with the starting conditions of the isocratic system.

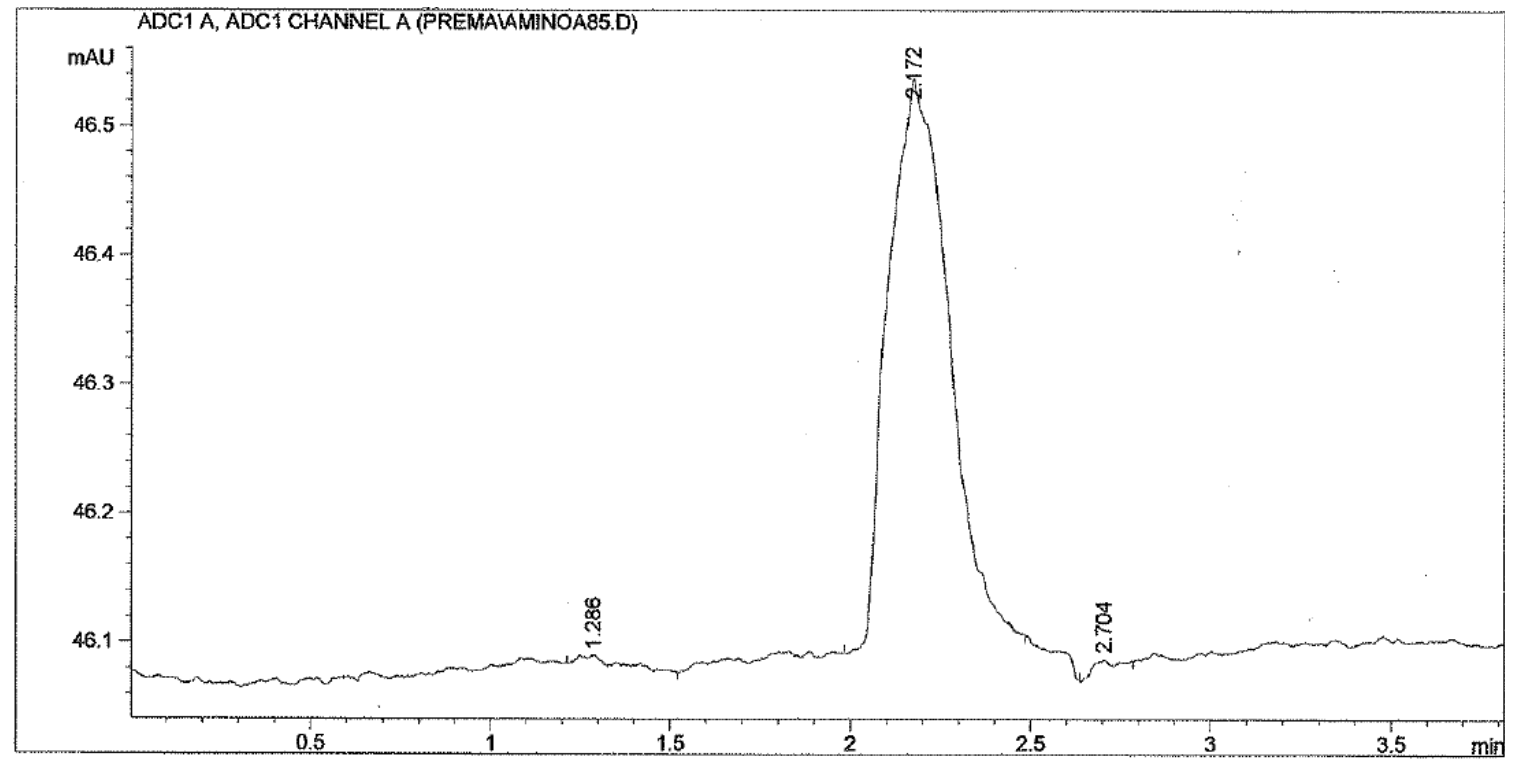

Figure 33. Chromatogram of phenylalanine on endcapped silica hydride stationary phase. Mobile phase: 40:60 water $+0.1 \%$ formic acid / acetonitrile $+0.1 \%$ formic acid. 


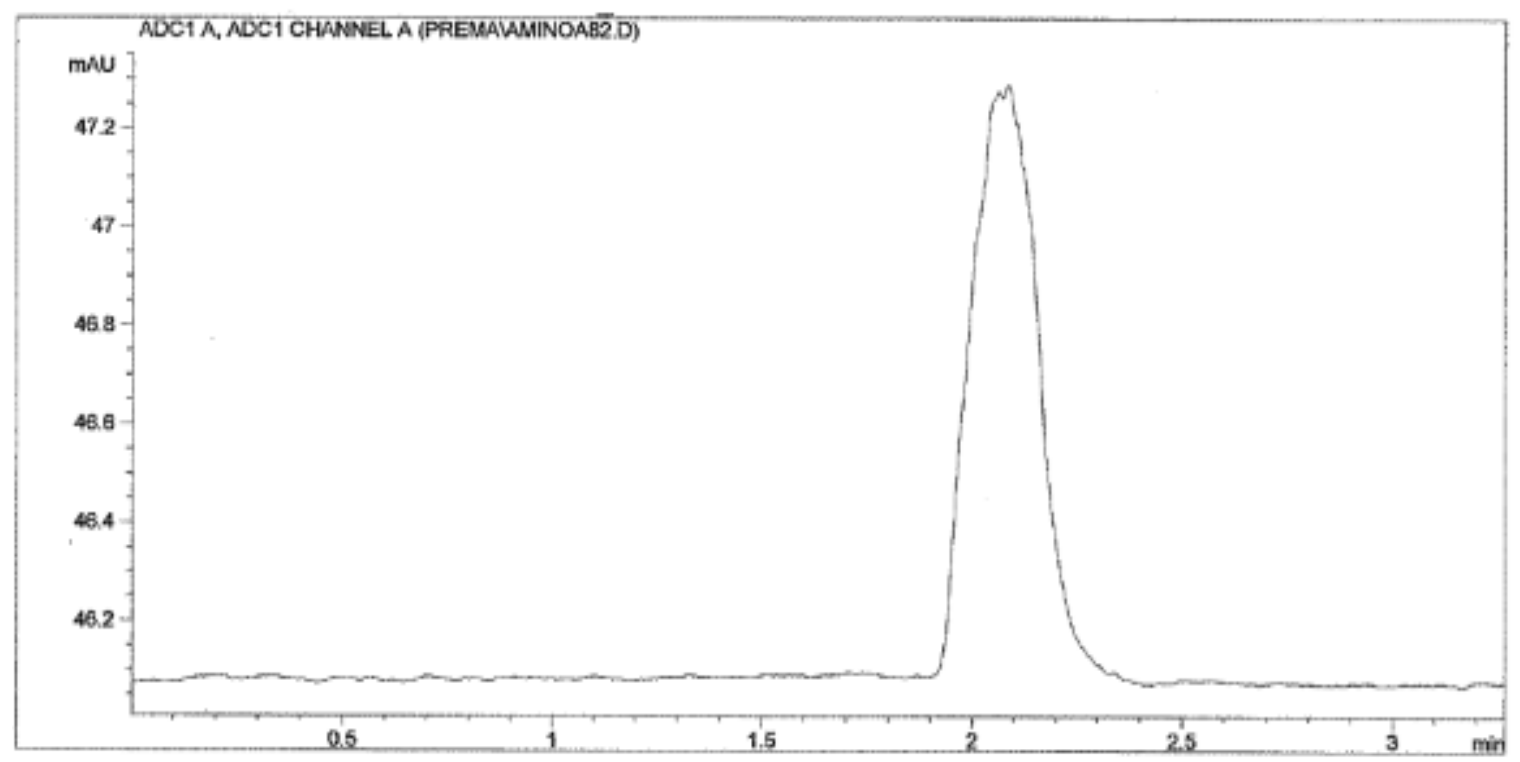

Figure 34. Chromatogram of phenylalanine on endcapped silica hydride phase. Mobile phase: $50: 50$ water $+0.1 \%$ formic acid / acetonitrile $+0.1 \%$ formic acid.

Phenylalanine was analyzed using $0.1 \%$ formic acid in water and $0.1 \%$ formic acid in acetonitrile under ANP isocratic conditions with an ELSD detector as shown in Figures 33 and 34. The compound was dissolved in 50:50 water:acetonitrile at a concentration of $0.5 \mathrm{mg} / \mathrm{mL}$. The compound was also analyzed using UV detection which is discussed in a subsequent section. Considerably more work has been done using UV detection in comparison to ELSD. 


\section{ANP and RP Behavior of Endcapped Si-H Using UV Detection}

The chromatographic characterization of the endcapped silica hydride column was done by measuring the retention behavior of the compounds discussed in the experimental section. The results were compared with the results of C8 (75 x $4.6 \mathrm{~mm}$ i.d) silica hydride column. The characterization of the column was done under aqueous normal phase mode as well as under reversed-phase mode. The parameters used for HPLC with UV detection were a flow rate $0.5 \mathrm{~mL} / \mathrm{min}$, an injection volume $5 \mu \mathrm{L}$, and the column temperature at ambient. The concentration of the samples was $0.5 \mathrm{mg} / \mathrm{mL}$ in 50:50 water and acetonitrile solution. The wavelengths used for the analysis were $254 \mathrm{~nm}$ and $220 \mathrm{~nm}$, and the column was maintained at the starting conditions of the gradient or isocratic system until a stable baseline was obtained. A water blank was run to eliminate any interference from the previous measurements. Raloxifene, dimethylthiourea, lipoic acid, triphenylphosphine, phenylalanine, niacinic acid, and lisinopril were analyzed using HPLC with a UV detector under isocratic and gradient conditions. The gradient program used for this research is shown in Table 7 and Table 8. The main goal of this research was to characterize the endcapped Si-H hydride column using aqueous normal phase and reversed-phase mode under isocratic and gradient conditions. 
Table 7. Mobile phase gradients used for reversed-phase analysis

\begin{tabular}{|c|c|c|c|c|c|}
\hline \multicolumn{3}{|c|}{ Gradient A } & \multicolumn{3}{c|}{ Gradient B } \\
\hline Time & Water with & Acetonitrile & Time & Water with & Acetonitrile \\
& 0.1\% TFA & with 0.1\% & $($ min) & 0.1\% TFA & with 0.1\% \\
& & TFA & & & TFA \\
\hline 0 & 100 & 0 & 0 & 95 & 5 \\
\hline 25 & 60 & 40 & 5 & 95 & 5 \\
\hline 35 & 30 & 70 & 35 & 5 & 95 \\
\hline 40 & 30 & 70 & 40 & 5 & 95 \\
\hline 45 & 100 & 0 & 45 & 95 & 5 \\
\hline 50 & 100 & 0 & 50 & 95 & 5 \\
\hline
\end{tabular}


Table 8. Mobile phase gradients used for reversed-phase analysis

\begin{tabular}{|c|c|c|c|c|c|}
\hline \multicolumn{3}{|c|}{ Gradient C } & \multicolumn{3}{|c|}{ Gradient D } \\
\hline $\begin{array}{l}\text { Time } \\
(\mathrm{min})\end{array}$ & $\begin{array}{c}\text { Water } \\
\text { with } 0.1 \% \\
\text { TFA }\end{array}$ & $\begin{array}{c}\text { Acetonitrile } \\
\text { with } 0.1 \% \\
\text { TFA }\end{array}$ & $\begin{array}{l}\text { Time } \\
\text { (min) }\end{array}$ & $\begin{array}{c}\text { Water } \\
\text { with } 0.1 \% \\
\text { TFA }\end{array}$ & $\begin{array}{c}\text { Acetonitrile } \\
\text { with } 0.1 \% \\
\text { TFA }\end{array}$ \\
\hline $\mathbf{0}$ & 75 & 25 & $\mathbf{0}$ & 75 & 25 \\
\hline 5 & 75 & 25 & 5 & 75 & 25 \\
\hline 30 & 55 & 45 & 30 & 50 & 50 \\
\hline 35 & 50 & 50 & 35 & $\mathbf{0}$ & 100 \\
\hline 40 & 5 & 95 & 40 & $\mathbf{0}$ & 100 \\
\hline 50 & 5 & 95 & 45 & 75 & 25 \\
\hline 55 & 75 & 25 & 50 & 75 & 25 \\
\hline 60 & 75 & 25 & & & \\
\hline
\end{tabular}




\section{a. Aqueous Normal Phase Behavior of Phenylalanine}

Phenylalanine was analyzed using aqueous normal phase isocratic conditions and concentration used was $0.5 \mathrm{mg} / \mathrm{mL}$ in a 50:50 water/acetonitrile solution. $220 \mathrm{~nm} \mathrm{UV}$ wavelength was selected and stabilized with the starting conditions of the isocratic system.

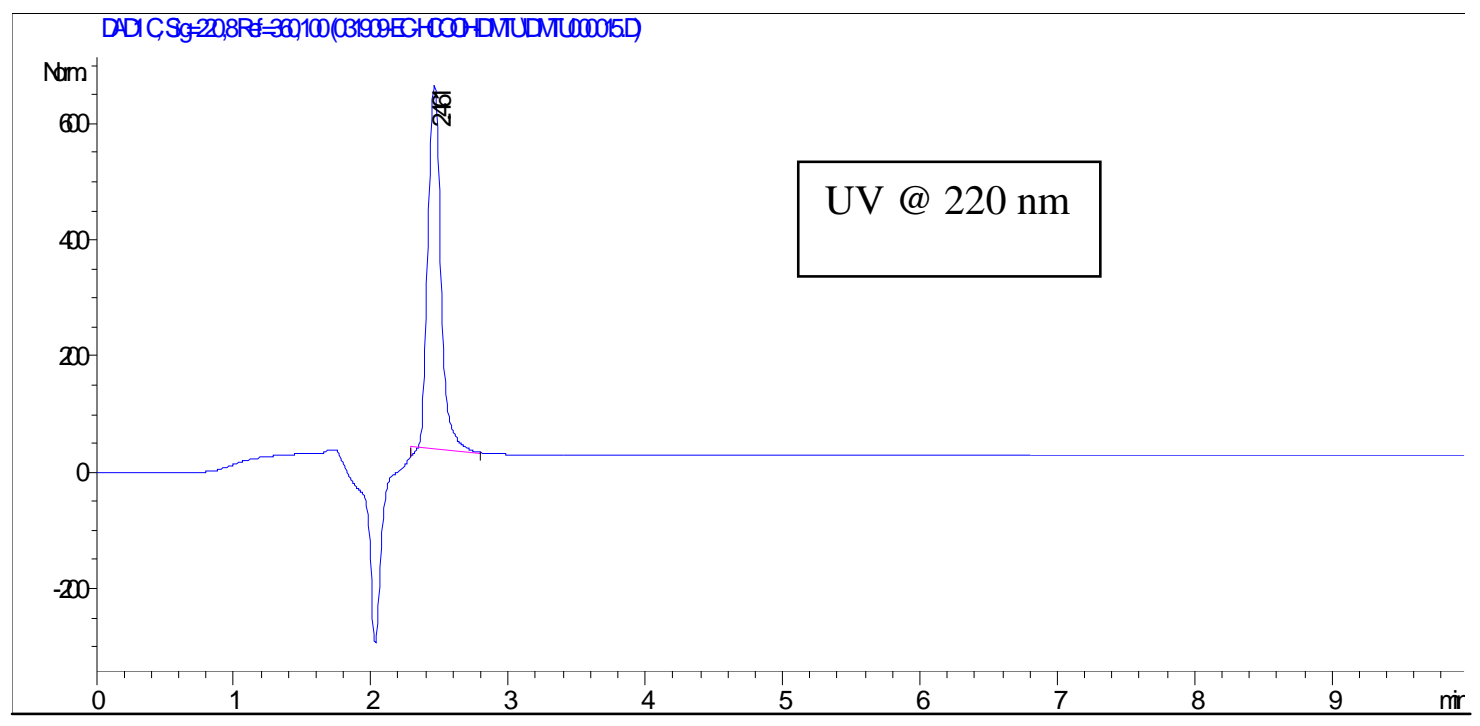

Figure 35. Chromatogram of phenylalanine on endcapped silica hydride stationary phase. Mobile phase: $40: 60$ water $+0.1 \%$ formic acid / acetonitrile $+0.1 \%$ formic acid. 


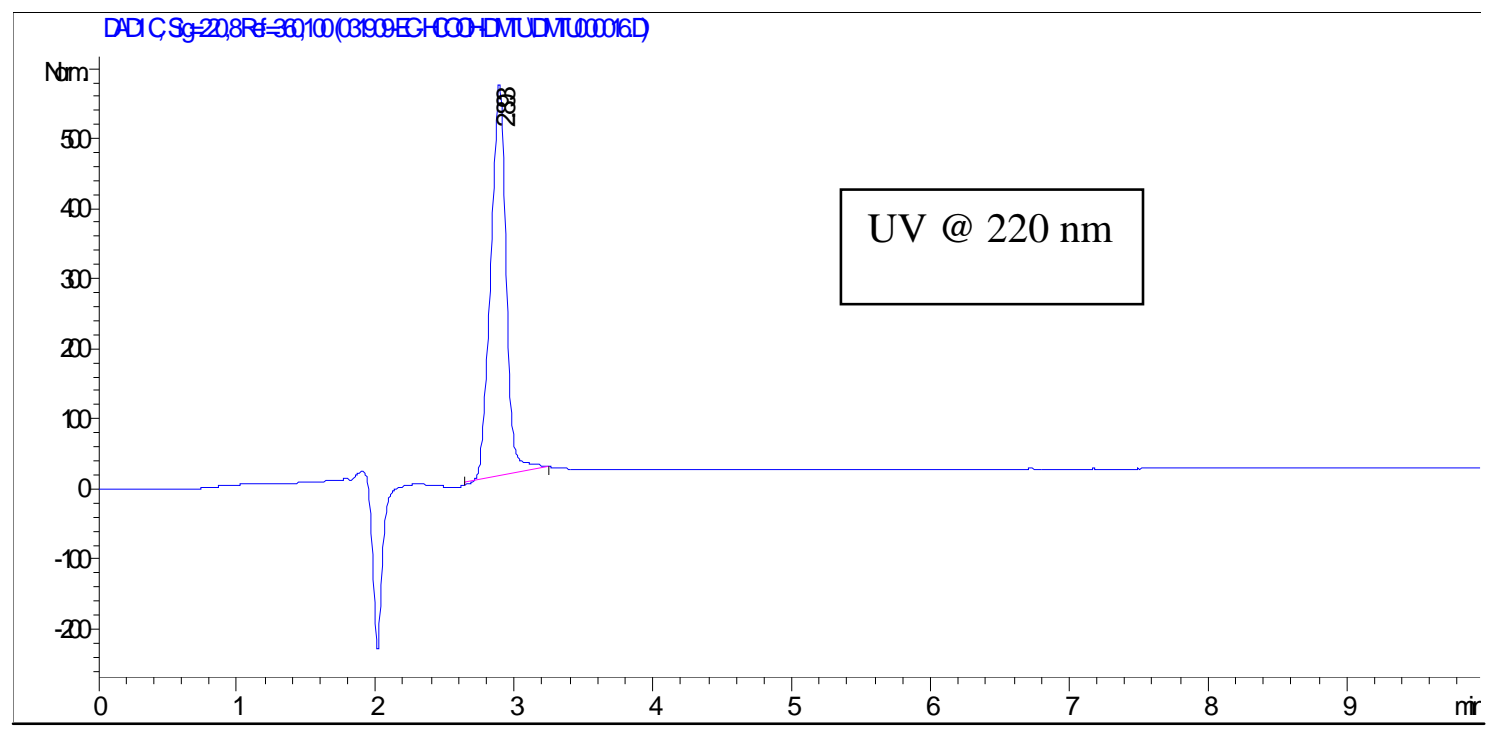

Figure 36. Chromatogram of phenylalanine on endcapped silica hydride stationary phase. Mobile phase: 30:70 water $+0.1 \%$ formic acid / acetonitrile $+0.1 \%$ formic acid. 


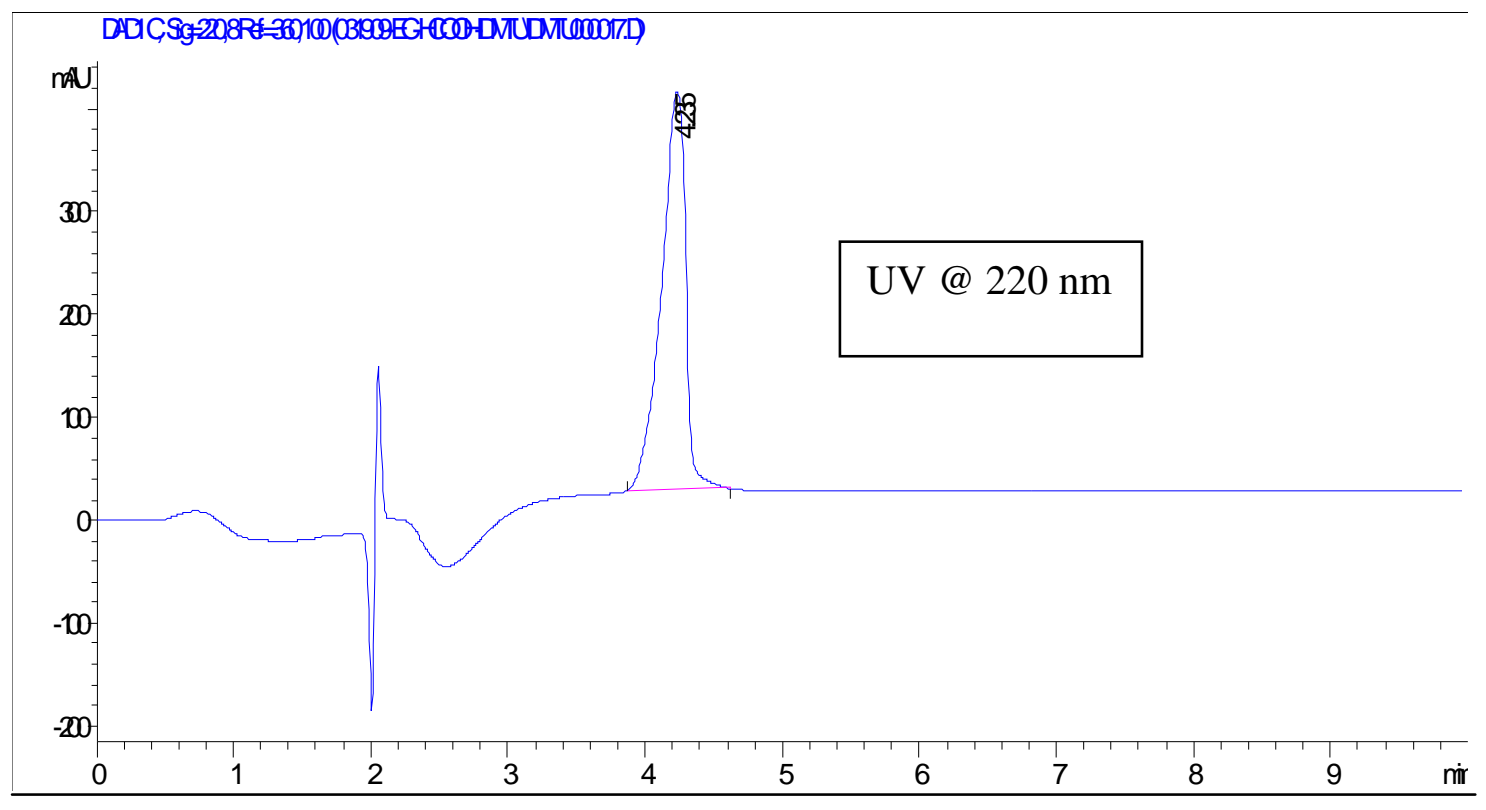

Figure 37. Chromatogram of phenylalanine on endcapped silica hydride stationary phase. Mobile phase: $20: 80$ water $+0.1 \%$ formic acid / acetonitrile $+0.1 \%$ formic acid.

Table 9. Summary of isocratic elution data for phenylalanine

\begin{tabular}{|c|c|c|}
\hline \multicolumn{3}{|c|}{ Phenylalanine Isocratic } \\
\hline \% Water + & \% Acetonitrile & Retention \\
0.1\% FA & $+0.1 \%$ FA & time (min) \\
\hline 40 & 60 & 2.5 \\
\hline 30 & 70 & 2.9 \\
\hline 20 & 80 & 4.2 \\
\hline
\end{tabular}


Phenylalanine was analyzed using aqueous normal phase isocratic conditions. The mobile phase used was $0.1 \%$ formic acid in water and $0.1 \%$ formic acid in acetonitrile as shown in Figures 35 to 37. The compound was dissolved in 50:50 water/acetonitrile at a concentration of $0.5 \mathrm{mg} / \mathrm{mL}$ and the detector used was UV. In ANP mode the retention of the compound increases as the percentage of acetonitrile increases as shown in Table 9.

\section{b. Aqueous Normal Phase Behavior of Niacinic Acid}

Niacinic acid was analyzed using aqueous normal phase isocratic conditions and concentration used was $0.5 \mathrm{mg} / \mathrm{mL}$ in a 50:50 water/acetonitrile solution. $220 \mathrm{~nm} \mathrm{UV}$ wavelength was selected and stabilized with the starting conditions of the isocratic system. 


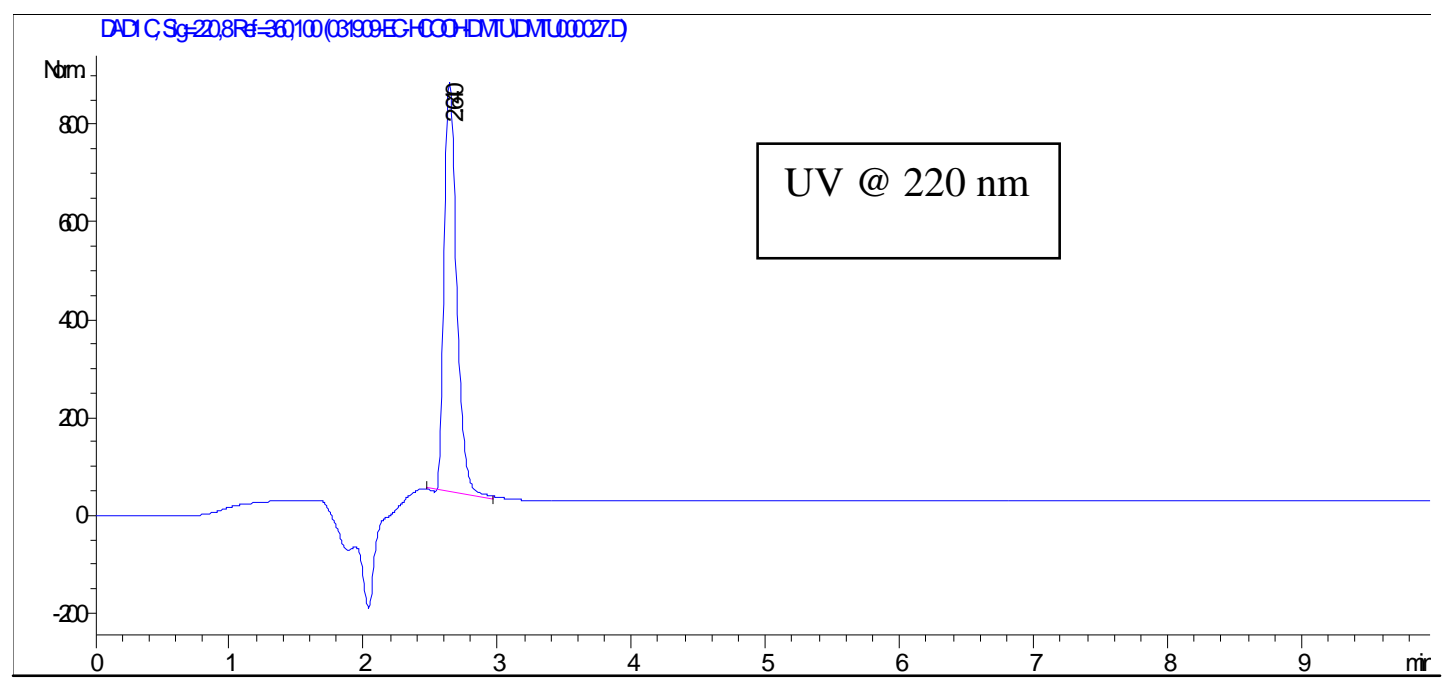

Figure 38. Chromatogram of niacinic acid on endcapped silica hydride stationary phase. Mobile phase: 40:60 water $+0.1 \%$ formic acid / acetonitrile $+0.1 \%$ formic acid.

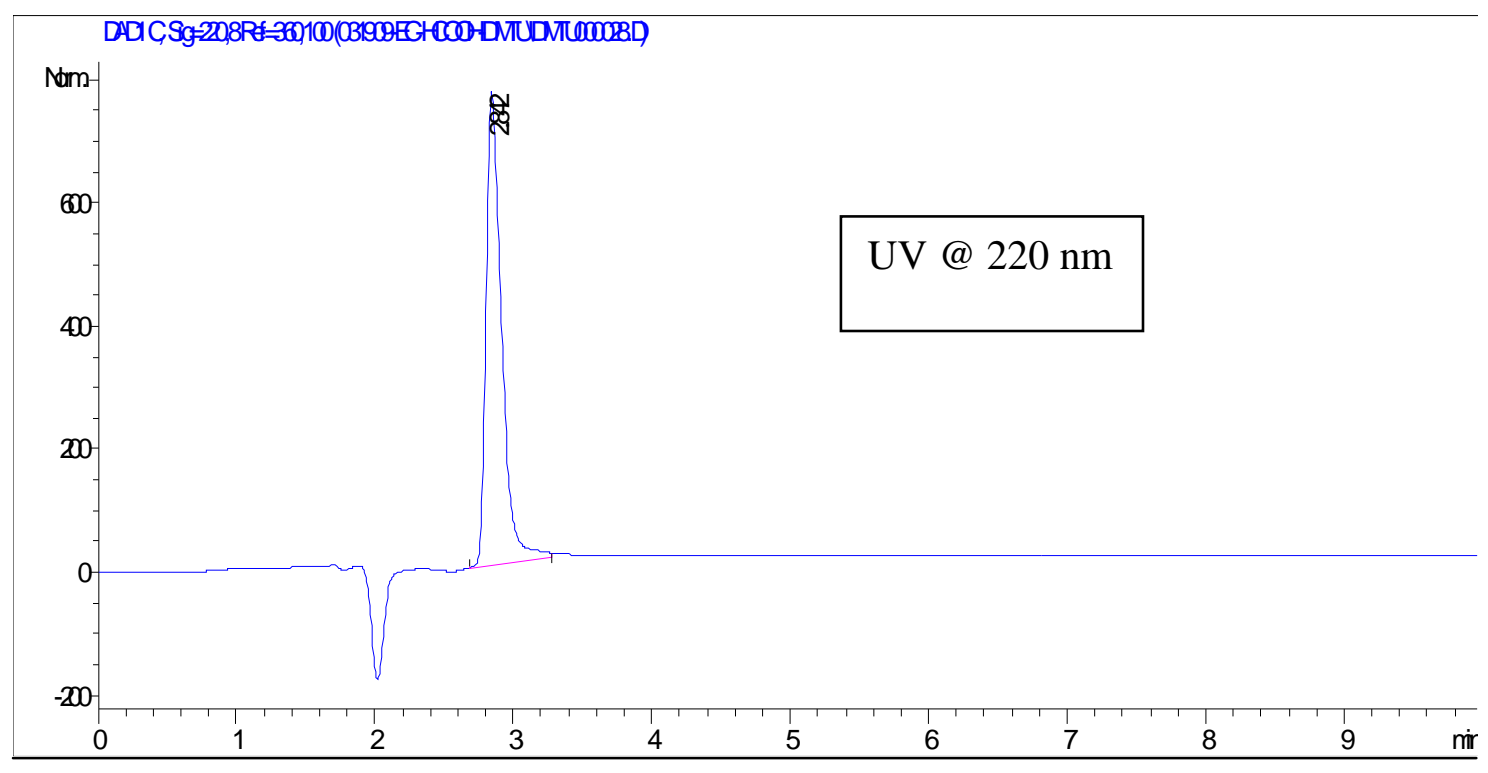

Figure 39. Chromatogram of niacinic acid on endcapped silica hydride stationary phase. Mobile phase: $30: 70$ water $+0.1 \%$ formic acid / acetonitrile $+\mathbf{0 . 1 \%}$ formic acid. 


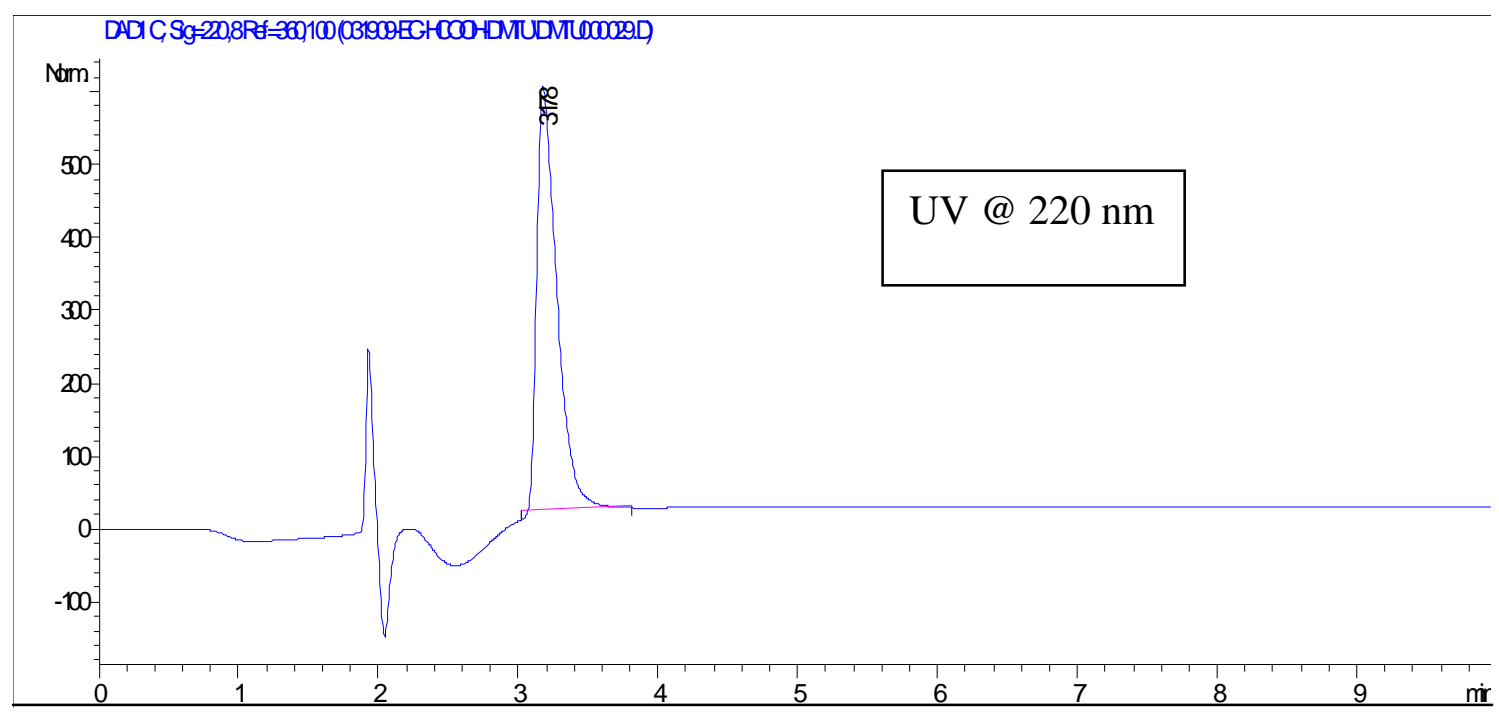

Figure 40. Chromatogram of niacinic acid on endcapped silica hydride stationary phase. Mobile phase: $20: 80$ water $+0.1 \%$ formic acid / acetonitrile $+0.1 \%$ formic acid.

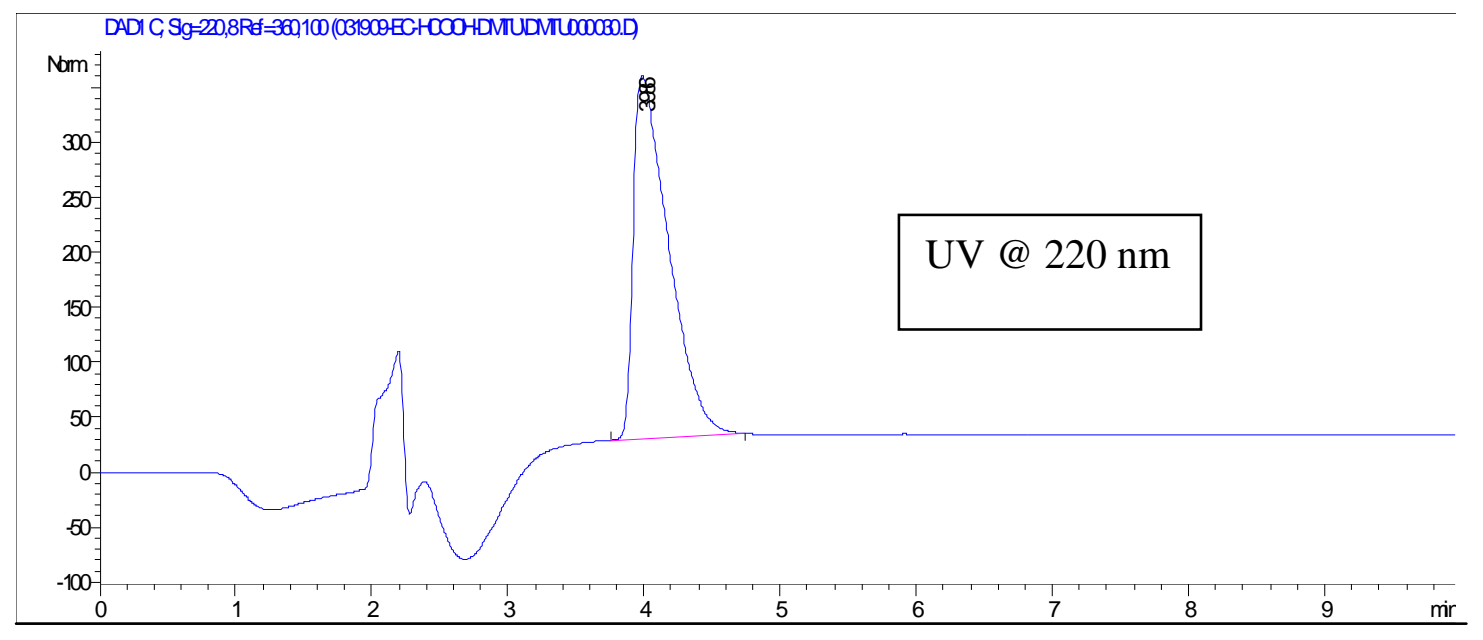

Figure 41. Chromatogram of niacinic acid on endcapped silica hydride stationary phase. Mobile phase: 10:90 water $+0.1 \%$ formic acid / acetonitrile $+\mathbf{0 . 1 \%}$ formic acid. 


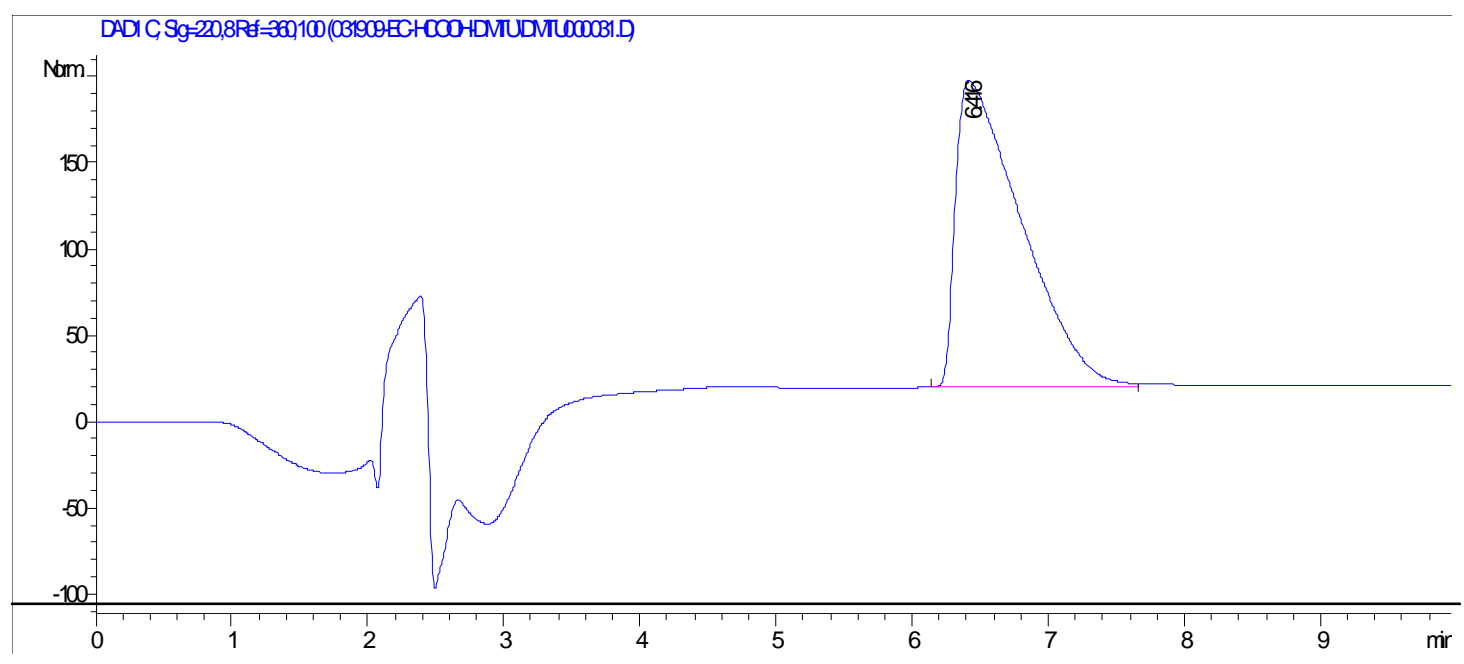

Figure 42. Chromatogram of niacinic acid on endcapped silica hydride stationary phase. Mobile phase: 5:95 water $+0.1 \%$ formic acid / acetonitrile $+0.1 \%$ formic acid.

Table 10. Summary of isocratic elution data for niacinic acid

\begin{tabular}{|c|c|c|}
\hline \multicolumn{3}{|c|}{ Niacinic Acid - Isocratic } \\
\hline \% Water + & \% Acetonitrile + & Retention \\
$0.1 \%$ FA & $0.1 \%$ FA & time (min) \\
\hline 40 & 60 & 2.2 \\
\hline 30 & 70 & 2.8 \\
\hline 20 & 80 & 3.2 \\
\hline 10 & 90 & 4 \\
\hline 5 & 95 & 6.4 \\
\hline
\end{tabular}


Niacinic acid was analyzed using UV detection under different isocratic conditions as shown in Figures 38 to 42 under aqueous normal phase conditions. As shown in Table 10, in ANP elution as the composition of acetonitrile increases the retention of the compound increases.

\section{c. Aqueous Normal Phase Behavior of Lisinopril}

Lisinopril was analyzed using aqueous normal phase isocratic conditions and concentration used was $0.5 \mathrm{mg} / \mathrm{mL}$ in a $50: 50$ water/acetonitrile solution.

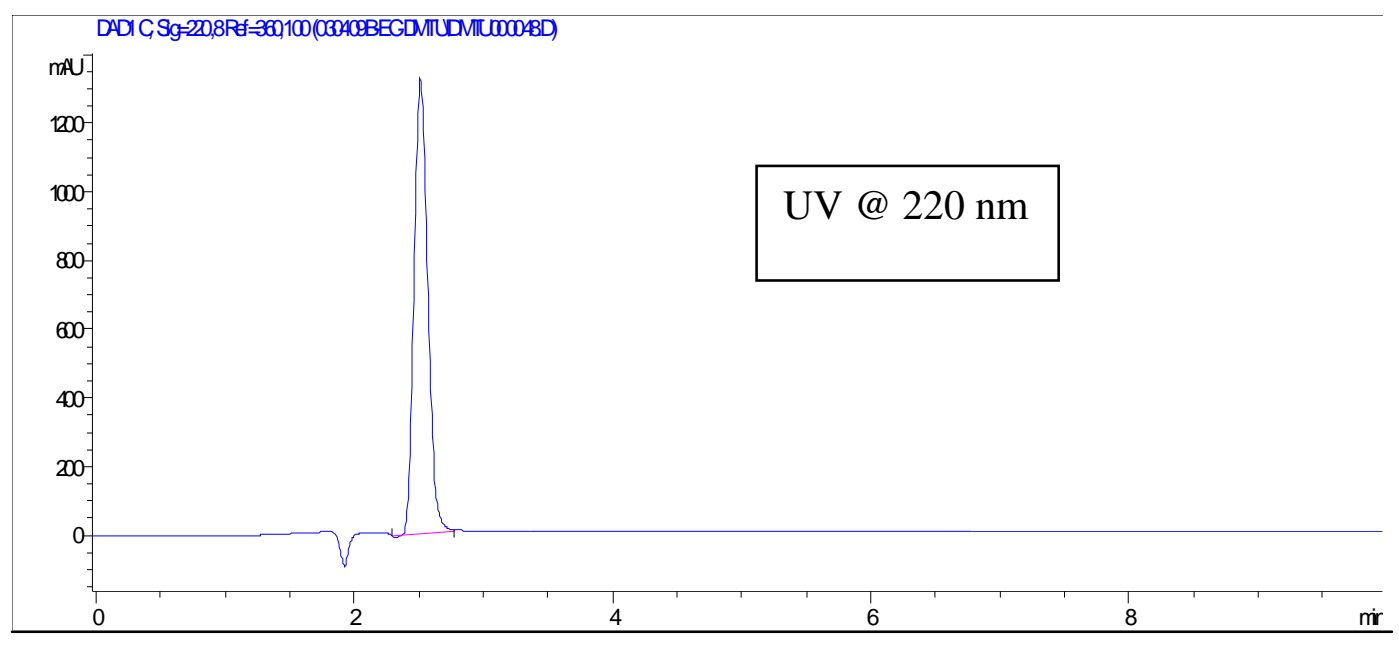

Figure 43. Chromatogram of lisinopril on endcapped silica hydride stationary phase. Mobile phase: $20: 80$ water $+0.1 \%$ trifluoroacetic acid / acetonitrile $+0.1 \%$ trifluoroacetic acid. 


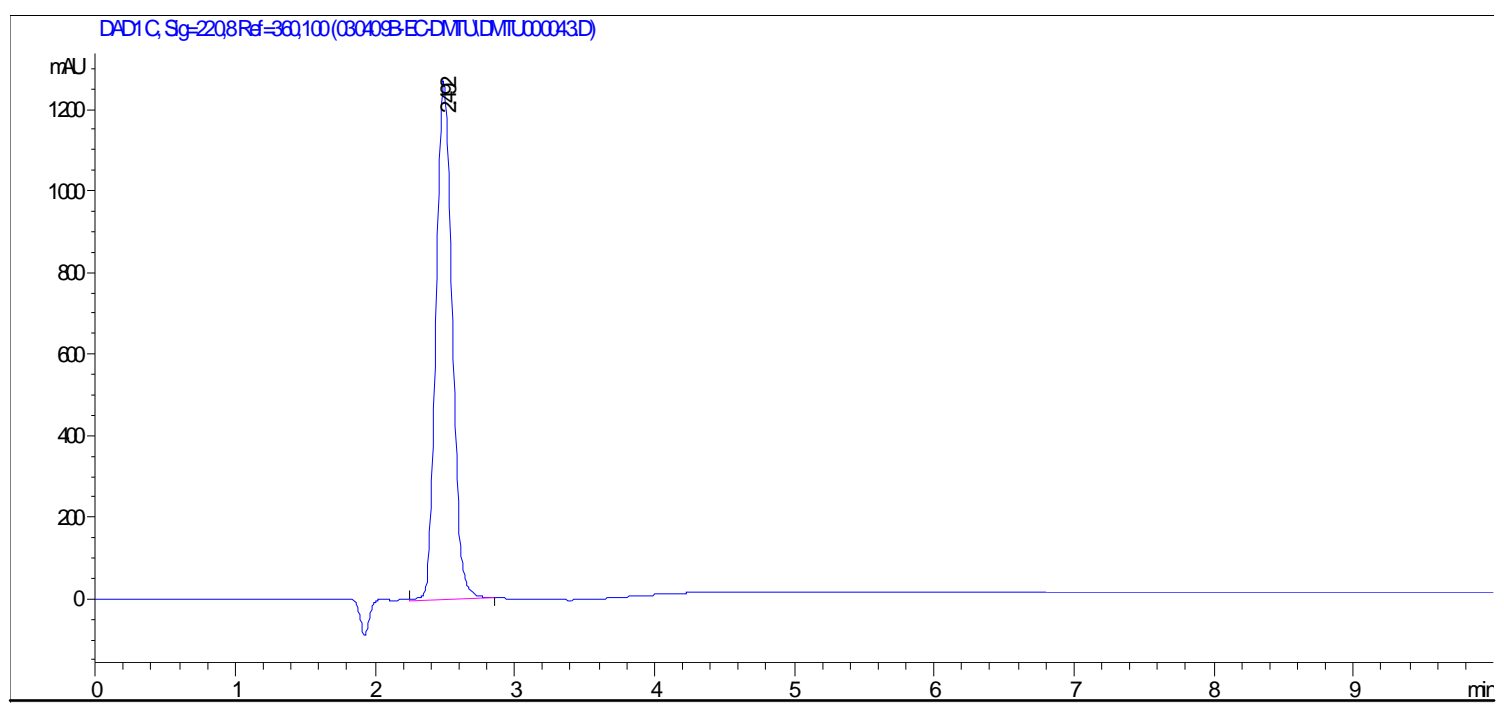

Figure 44. Chromatogram of lisinopril on endcapped silica hydride stationary phase. Mobile phase: 10:90 water $+\mathbf{0 . 1 \%}$ trifluoroacetic acid / acetonitrile $+\mathbf{0 . 1 \%}$ trifluoroacetic acid.

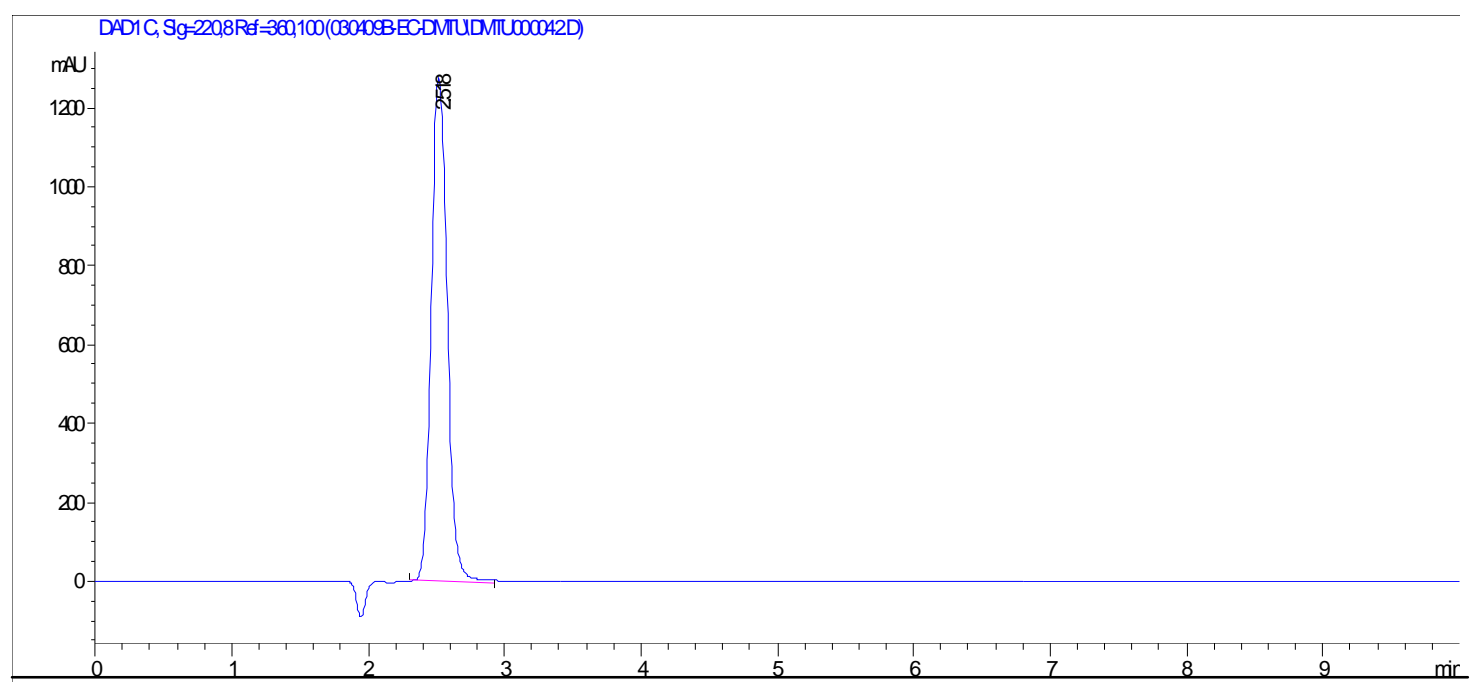

Figure 45. Chromatogram of lisinopril on endcapped silica hydride stationary phase. Mobile phase: 5:95 water $+0.1 \%$ trifluoroacetic acid / acetonitrile $+0.1 \%$ trifluoroacetic acid. 
Table 11. Summary of isocratic elution data for lisinopril

\begin{tabular}{|c|c|c|}
\hline \multicolumn{3}{|c|}{ Lisinopril - Isocratic } \\
\hline \% Water + 0.1\% FA & \% Acetonitrile + & Retention \\
& 0.1\% FA & time (min) \\
\hline 50 & 50 & 2.7 \\
\hline 30 & 70 & 2.0 \\
\hline 20 & 80 & 2.2 \\
\hline 10 & 90 & 2.5 \\
\hline 5 & 95 & 2.5 \\
\hline
\end{tabular}

Lisinopril was analyzed using UV detection under different isocratic conditions.

The Figures from 43 to 45 show aqueous normal phase elution and as shown in Table 11 as the composition of acetonitrile increase the retention of the compound increases above a composition of $50 \%$ acetonitrile. This data obtained confirmed the aqueous normal phase behavior of lisinopril. 


\section{d. ANP Separation of Two Compounds Using an Isocratic Method}

Lisinopril and lipoic acid was analyzed using aqueous normal phase isocratic conditions and concentration used was $0.5 \mathrm{mg} / \mathrm{mL}$ in a $50: 50$ water/acetonitrile solution.

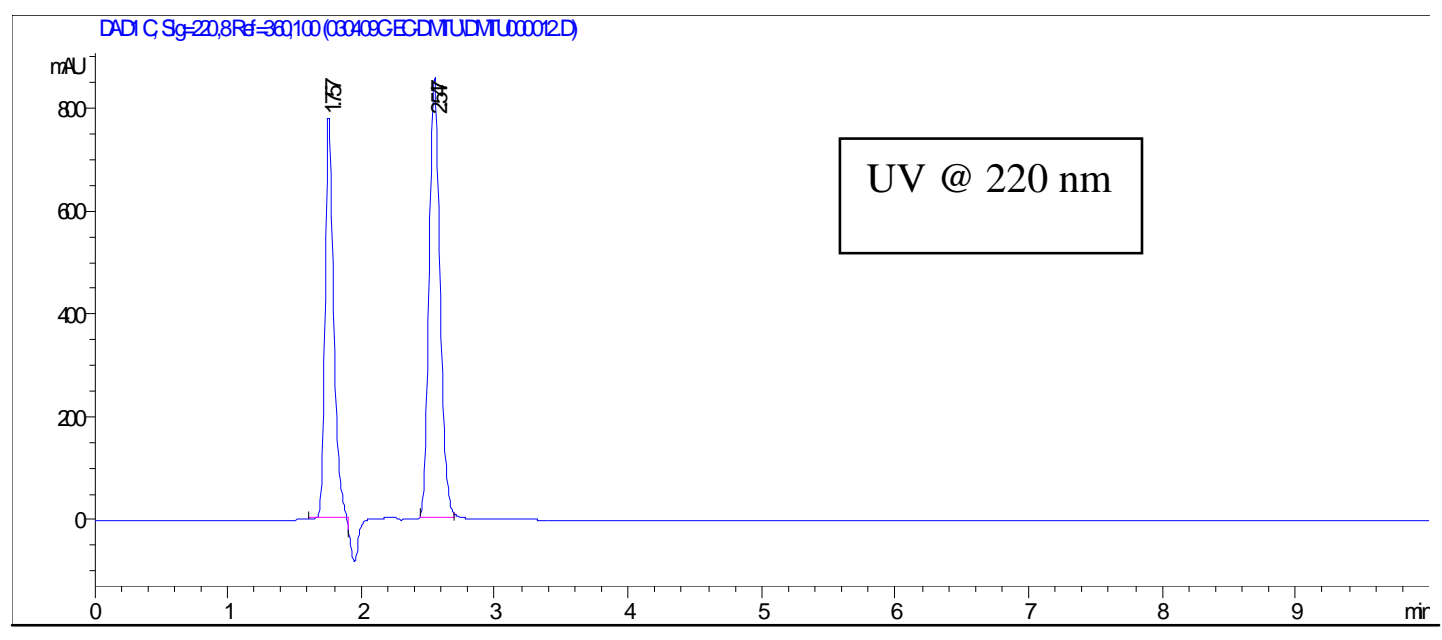

Figure 46. ANP isocratic separation of lipoic acid and lisinopril. Mobile phase: 95:5 acetonitrile/water $+\mathbf{0 . 1 \%}$ TFA. The peak at $1.75 \mathrm{~min}$ is for lipoic acid and the peak at $2.547 \mathrm{~min}$ is for lisinopril. 


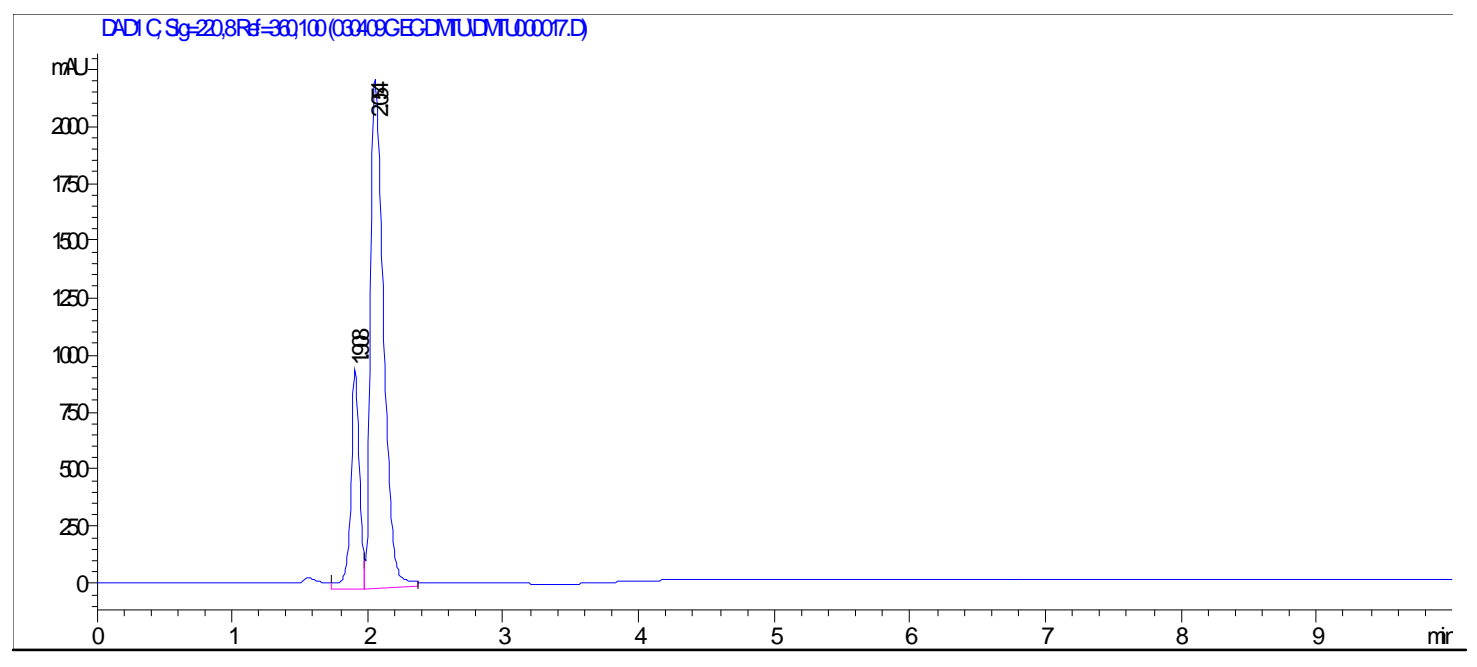

Figure 47. ANP isocratic separation of dimethylthiourea and raloxifene. Mobile phase: 95:5 acetonitrile/water $+0.1 \%$ TFA. The peak at $1.978 \mathrm{~min}$ is for raloxifene and the peak at $\mathbf{2 . 5 4 7} \mathrm{min}$ is for dimethylthiourea.

Two-compound mixture was tested on the endcapped silica hydride column to determine whether it was be possible to separate them either gradient or isocratic conditions. The first mixture analyzed was lisinopril and lipoic acid as shown in Figure 46. A second mixture analyzed was raloxifene and dimethylthiourea as shown in Figure 47; both mixtures were tested for ANP behavior. The samples were analyzed using UV detection under isocratic conditions. The polar compounds dimethylthiourea and lisinopril were retained longer than the nonpolar compounds raloxifene and lipoic acid, as expected under aqueous normal phase conditions. This was a very good example illustrating typical aqueous normal phase behavior in which nonpolar compounds elute before polar compounds. The polar compounds were retained longer and retention 
decreased as the percentage of polar solvent was increased. Therefore it can be confirmed that endcapped $\mathrm{Si}-\mathrm{H}$ showed good aqueous normal phase behavior.

\section{e. RP Behavior of Endcapped Si-H Using UV Detection Under Isocratic and Gradient Conditions}

In reversed-phase gradient separation the composition of the organic component is increased with respect to time. Nonpolar compounds are preferentially separated in this chromatographic mode.

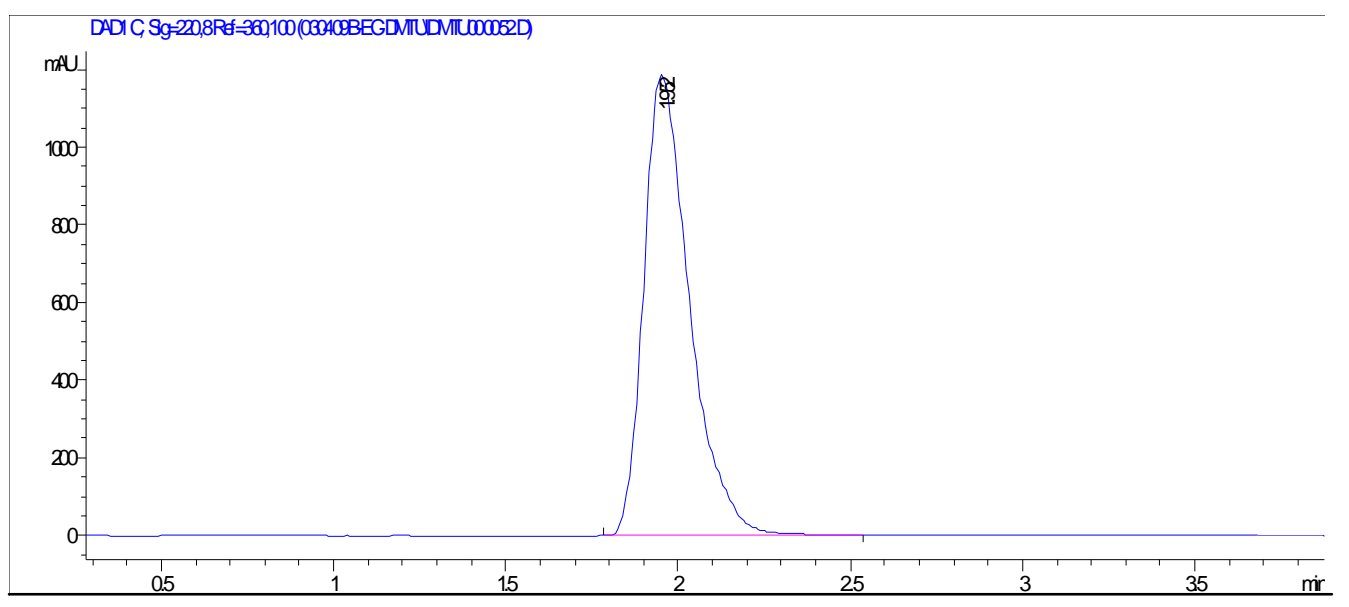

Figure 48. Chromatogram of lisinopril in reversed-phase mode on the endcapped silica hydride phase. Mobile phase: 75:25 water/acetonitrile $+0.1 \%$ TFA . 


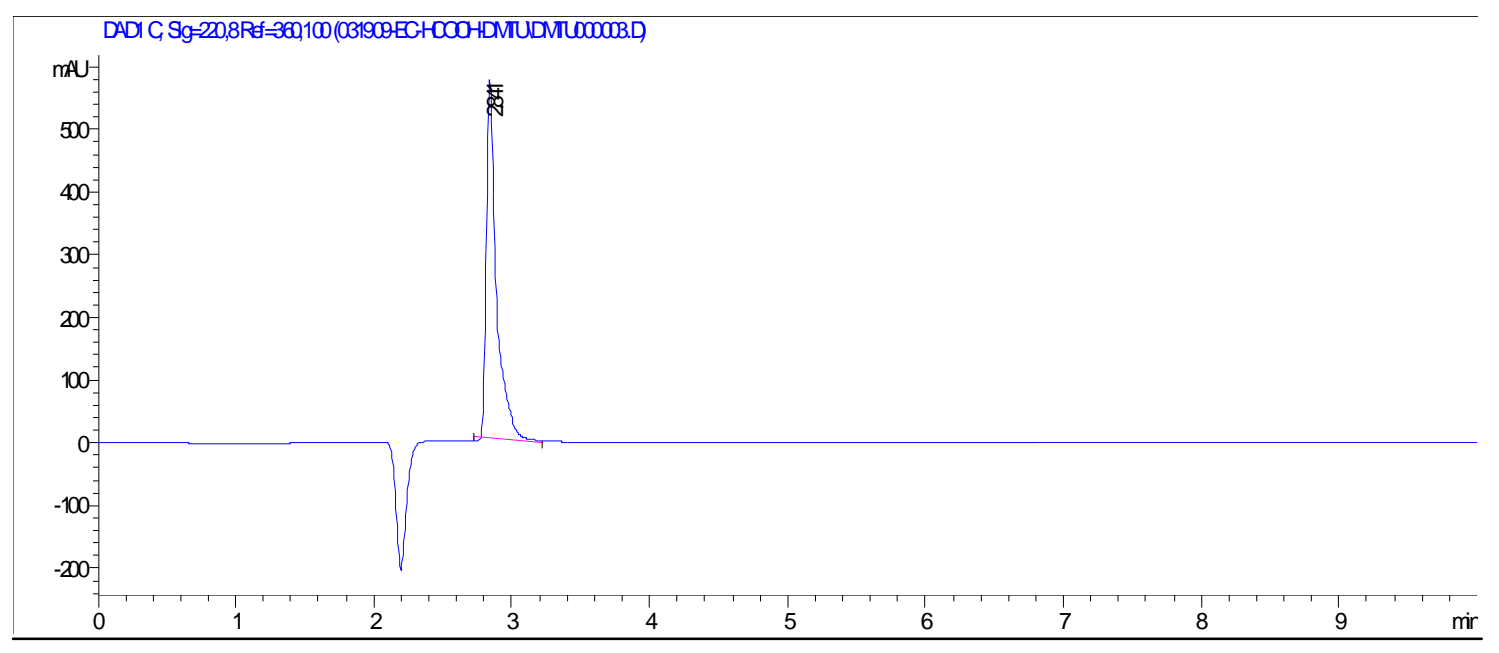

Figure 49. Chromatogram of dimethylthiourea in reversed-phase mode on endcapped silica hydride column using $100 \%$ water $+0.1 \%$ TFA.

Lisinopril and dimethylthiourea were measured using isocratic reversed-phase conditions as shown in Figures 48 and 49 and detected using UV detection at $220 \mathrm{~nm}$. As mentioned earlier silica hydride columns have the ability to separate both polar and nonpolar compounds. Endcapping the silica hydride enhances the hydrophobicity which will be discussed in a later section. 


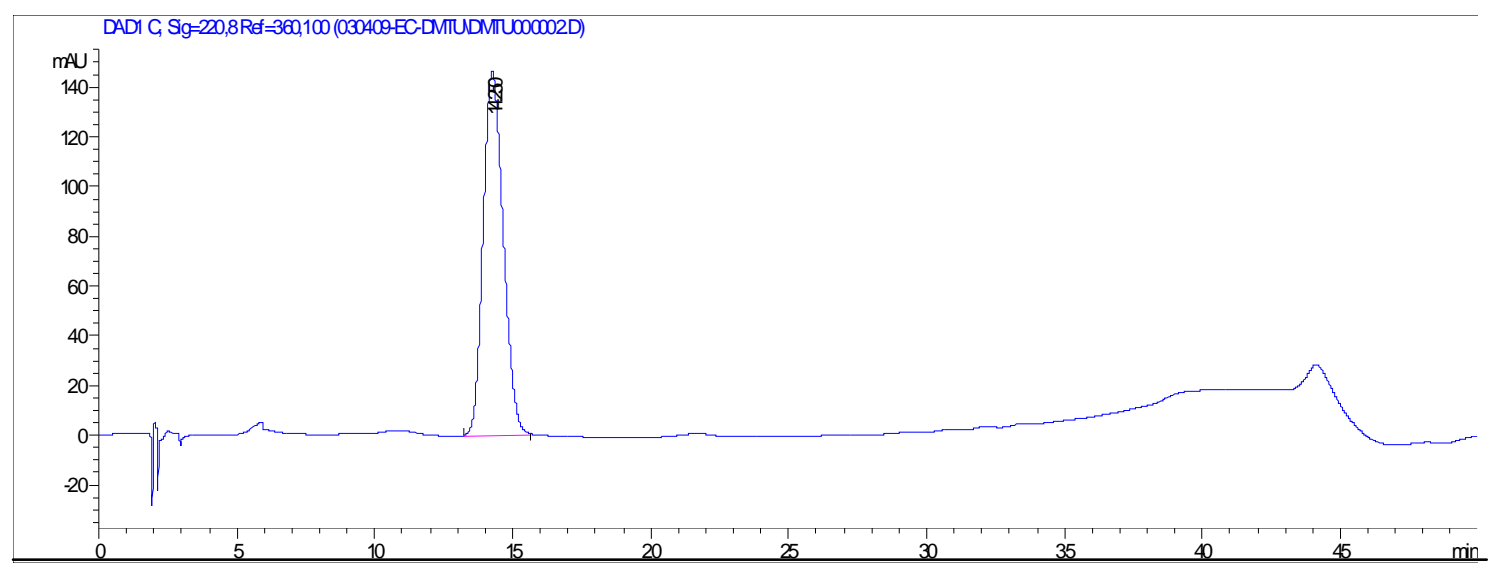

Figure 50. Chromatogram of raloxifene on the endcapped silica hydride column in reversed-phase mode using Gradient A shown in Table 7

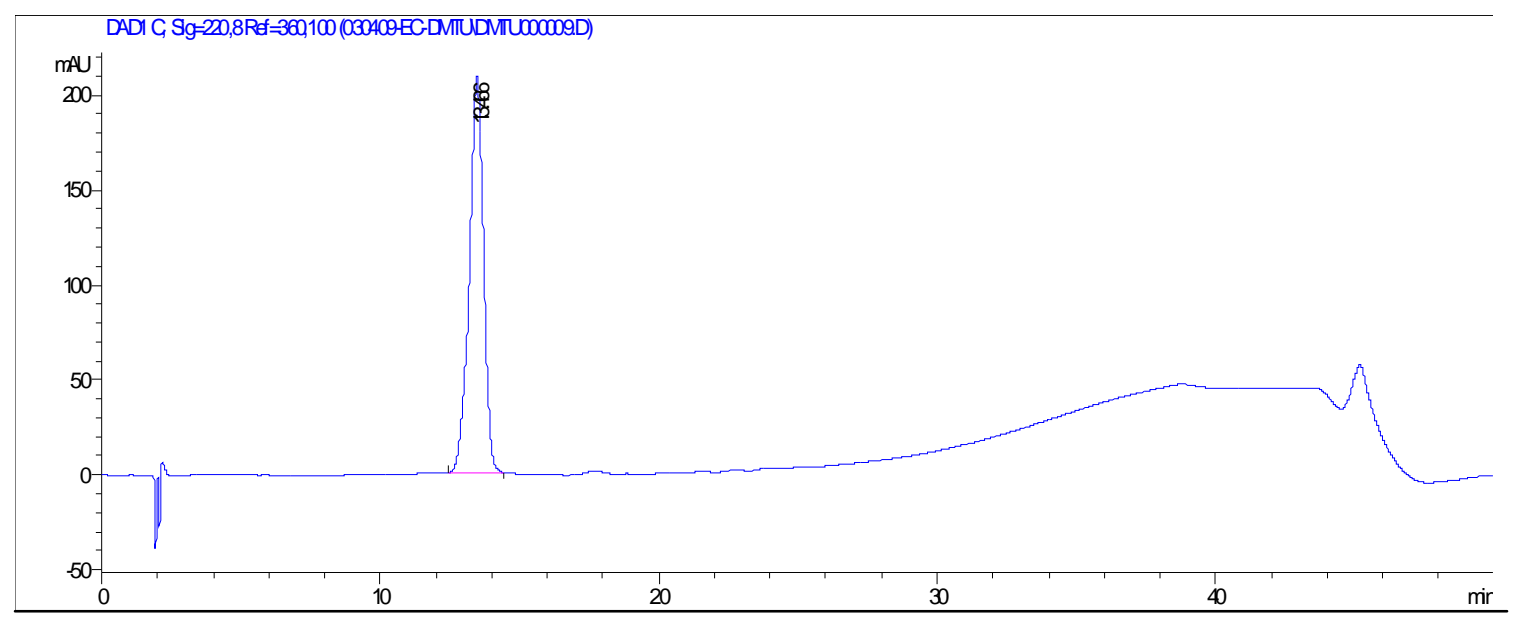

Figure 51. Chromatogram of raloxifene on the endcapped silica hydride column in reversed-phase mode using Gradient B shown in Table 7.

Raloxifene was eluted using Gradients A and B shown in Table 7 and the chromatograms are shown in Figures 50 and 51. The mobile phase used for this analysis 
was $0.1 \%$ TFA in water and $0.1 \%$ TFA in acetonitrile having flow rate of $0.5 \mathrm{~mL} / \mathrm{min}$. The concentration of the sample was $0.1 \mathrm{mg} / \mathrm{mL}$ dissolved in water. Figures 50 and 51 show the enhancement of the retention capabilities of the endcapped column for nonpolar compounds under reversed-phase conditions. The result was comparable with the $\mathrm{C} 8$ data which will be discussed in a subsequent section. The detector used for this data is UV at a wavelength of $220 \mathrm{~nm}$.

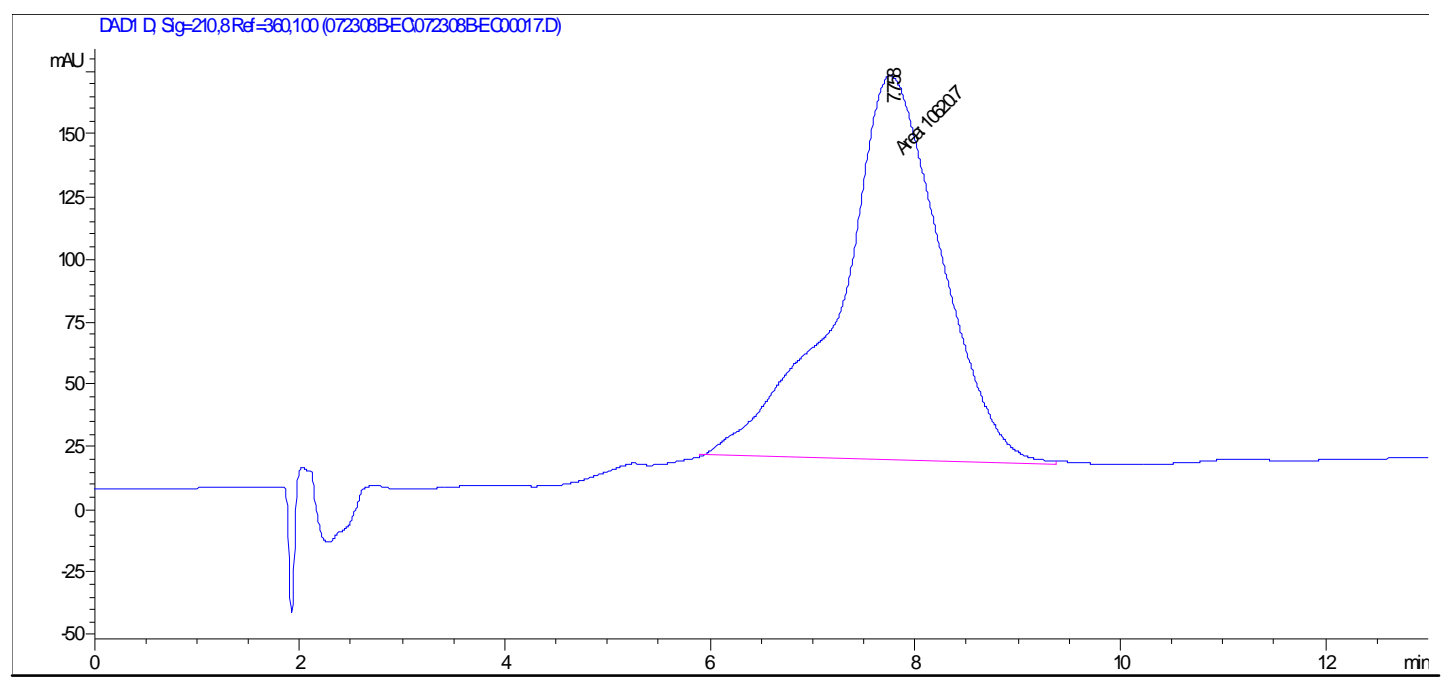

Figure 52. Chromatogram of lipoic acid on the endcapped silica hydride column in reversed-phase mode using Gradient A shown in Table 7.

Lipoic acid was eluted using Gradient A and the mobile phase used for this analysis was $0.1 \%$ TFA in water and $0.1 \%$ TFA in acetonitrile as shown in Figure 52. The peak shape was not symmetric under this gradient condition but it eluted at 8 min which was a good retention for the compound. 


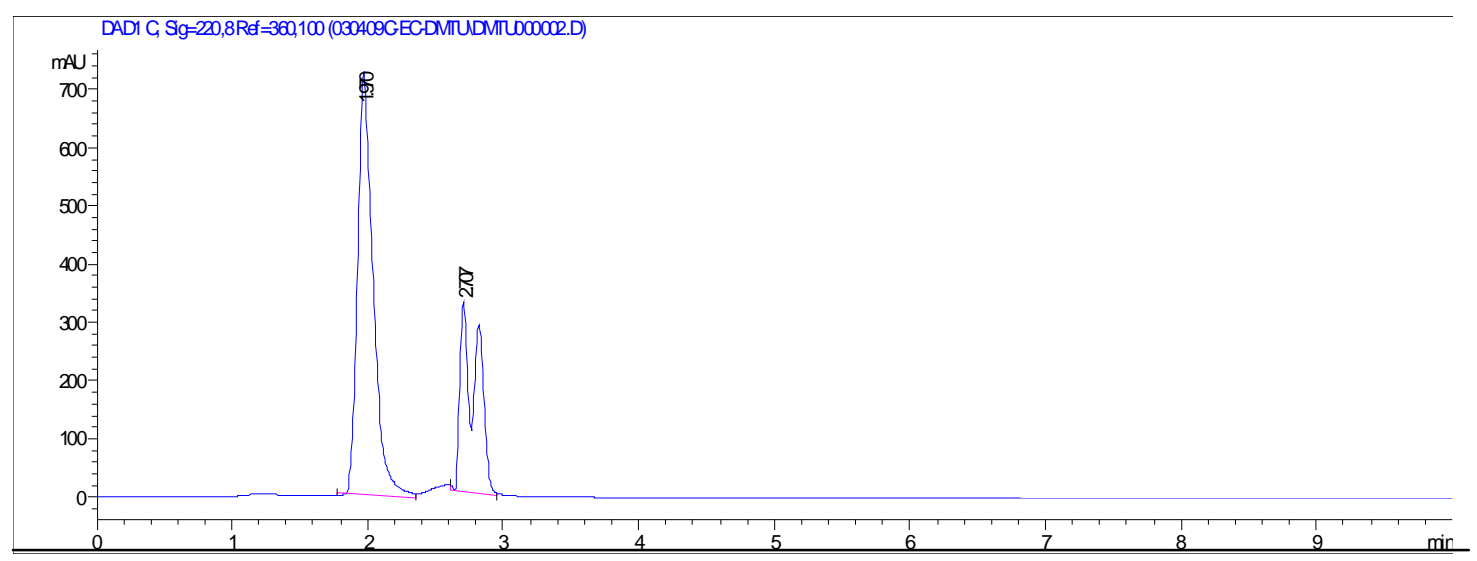

Figure 53. Reversed-phase isocratic separation of lisinopril and lipoic acid. The peak at $1.90 \mathrm{~min}$ is lisinopril and the peak at $2.8 \mathrm{~min}$ is lipoic acid. The isocratic composition used is 75:25 water/ acetonitrile $+0.1 \%$ TFA.

The lisinopril and lipoic acid sample mixture were separated using a reversedphase isocratic condition as shown in Figure 53. The mobile phase flow rate was 0.5 $\mathrm{mL} / \mathrm{min}$ and with UV detection at $220 \mathrm{~nm}$. Lisinopril being polar compared to lipoic acid eluted at $1.97 \mathrm{~min}$ and lipoic acid eluted at $2.8 \mathrm{~min}$. Lipoic acid is a stereoisomer, so it is possible that the isomers are being separated under the given conditions. In order to determine whether the endcapped silica hydride column can be used for the separation of stereoisomers, more work needed to be done using different isomeric compounds. 


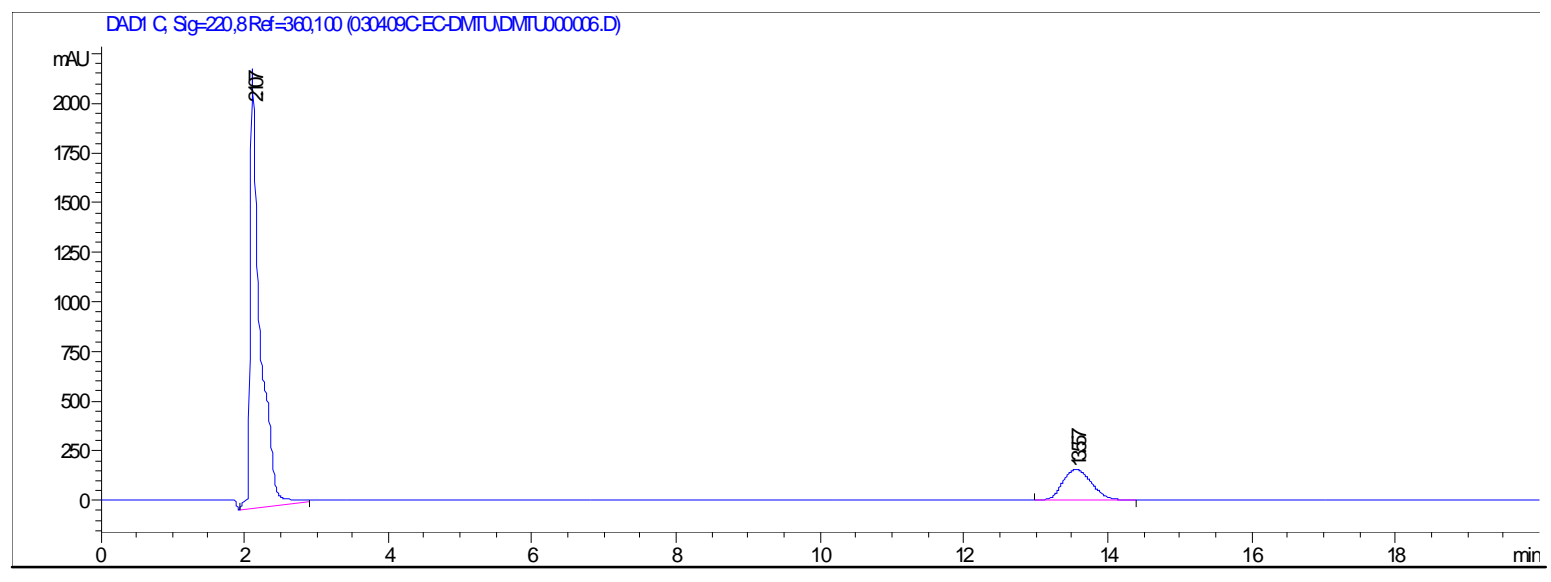

Figure 54. Reversed-phase gradient separation of dimethylthiourea and raloxifene. The peak at $\mathbf{2 . 0 7} \mathrm{min}$ is for dimethylthiourea and the peak $\mathbf{1 3 . 5 5 7} \mathbf{m i n}$ is for raloxifene. Gradient B used for the separation is shown in Table 7.

Raloxifene and dimethylthiourea were separated under reversed-phase conditions as shown in Figure 54. The gradient used for the separation was Gradient B shown in Table 7. The mobile phase flow rate was $0.5 \mathrm{~mL} / \mathrm{min}$ with UV detection at $220 \mathrm{~nm}$. Dimethylthiourea being very polar eluted at the beginning of the gradient and raloxifene being very nonpolar eluted at the latter part of the gradient.

From the results of this study, it can be interpreted that endcapping the silica hydride stationary phase with trimethylchlorosilane and hexamethyldisilazane increases the ability of the stationary phase for nonpolar compound separation. The separation between the compounds occurs by the hydrophobic interaction between the analytes and the stationary phase. The data obtained was comparable with the results obtained from 
the $\mathrm{C} 8$ column which will be discussed in a subsequent section. This confirms the fact that endcapping the silica hydride stationary phase increases the hydrophobic capabilities which can be used for the separation of nonpolar compounds.

As mentioned earlier that after silica modifications with TES there are some residual silanol groups present on the surface which may interact with the analyte molecule. This interaction might result in peak tailing, band broadening, and poor separation of basic compounds. One goal of this research was to reduce the amount of residual silanol groups on silica, and to prevent the remaining silanol group interaction with the samples of interest by endcapping $\mathrm{Si}-\mathrm{OH}$. 


\section{ANP and RP Behavior of Si-H based C8 Column Using UV Detection}

\section{a. Aqueous Normal Phase Behavior}

Phenylalanine, raloxifene, dimethylthiourea, and lipoic acid were analyzed under aqueous normal phase conditions on Si-H based C8 column as shown in Figures 55 to 60. The concentrations of the samples are $0.5 \mathrm{mg} / \mathrm{mL}$ and the detector used was UV.

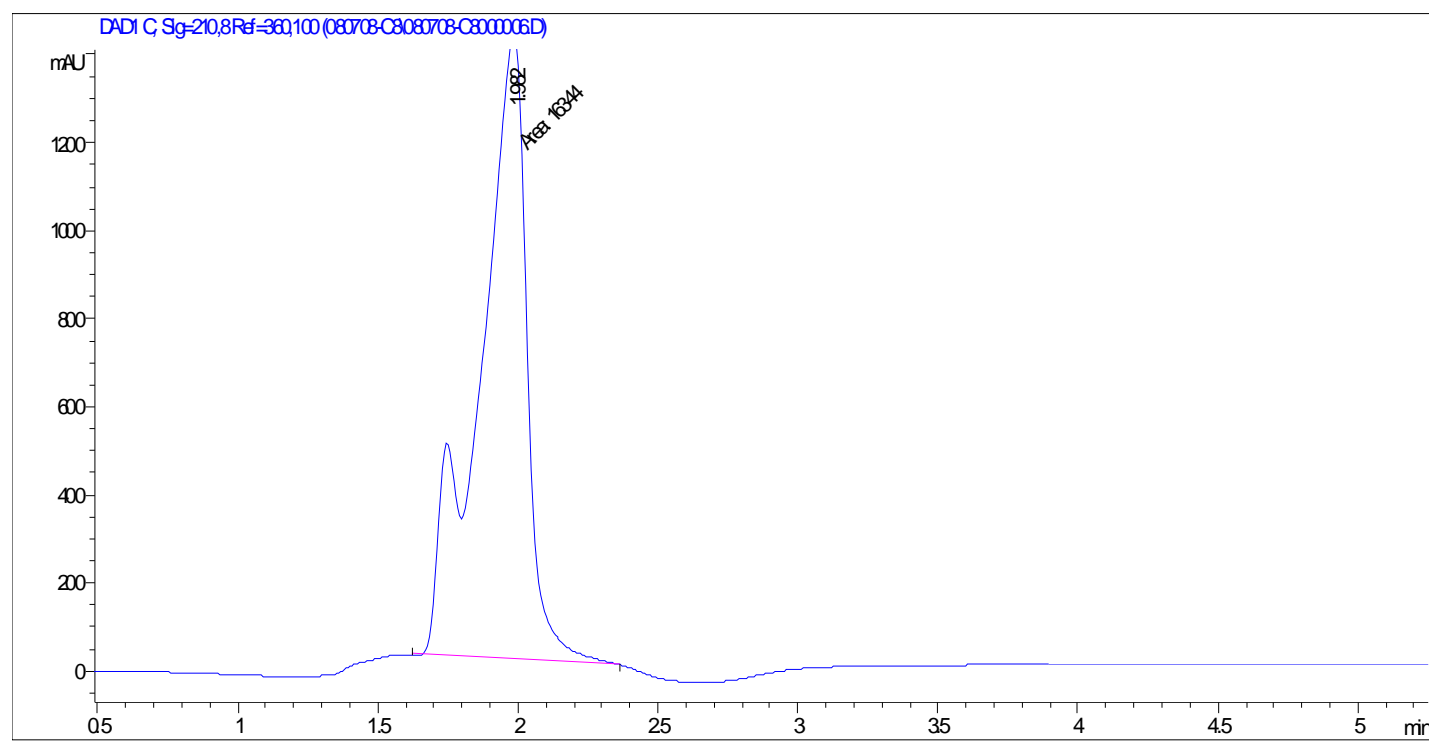

Figure 55. ANP isocratic retention of phenylalanine on silica hydride-based C8 column. The isocratic composition used is 60:40 acetonitrile/water $+0.1 \%$ TFA. 
Table 12. Summary of isocratic elution data for Phenylalanine

\begin{tabular}{|c|c|c|}
\hline \multicolumn{3}{|c|}{ Phenylalanine-Isocratic condition } \\
\hline Water + 0.1\% TFA & Acetonitrile + 0.1\% & RT (min) \\
& TFA & \\
\hline 50 & 50 & 1.7 \\
\hline 40 & 60 & 2 \\
\hline 5 & 95 & 1.9 \\
\hline
\end{tabular}

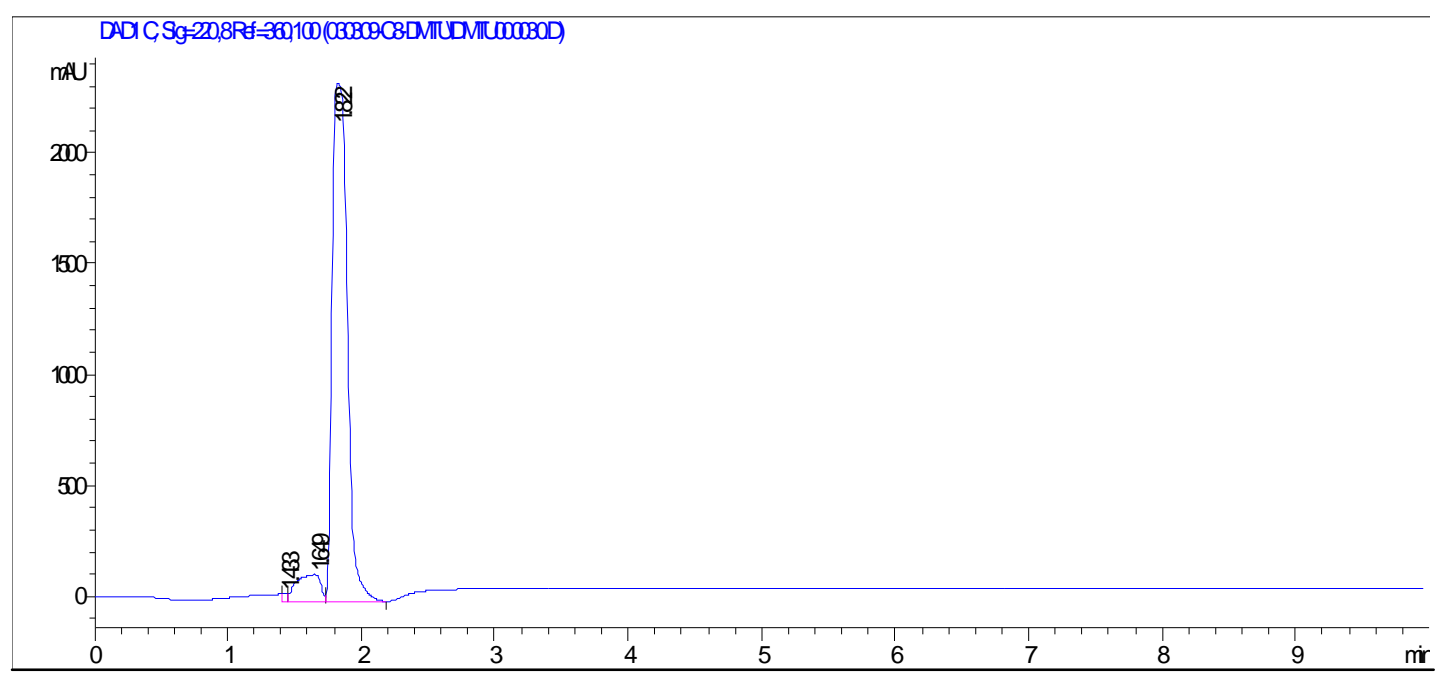

Figure 56. ANP isocratic retention of dimethylthiourea on silica hydride-based C8 column. The isocratic composition used is $90: 10$ acetonitrile/water $+0.1 \%$ TFA. 


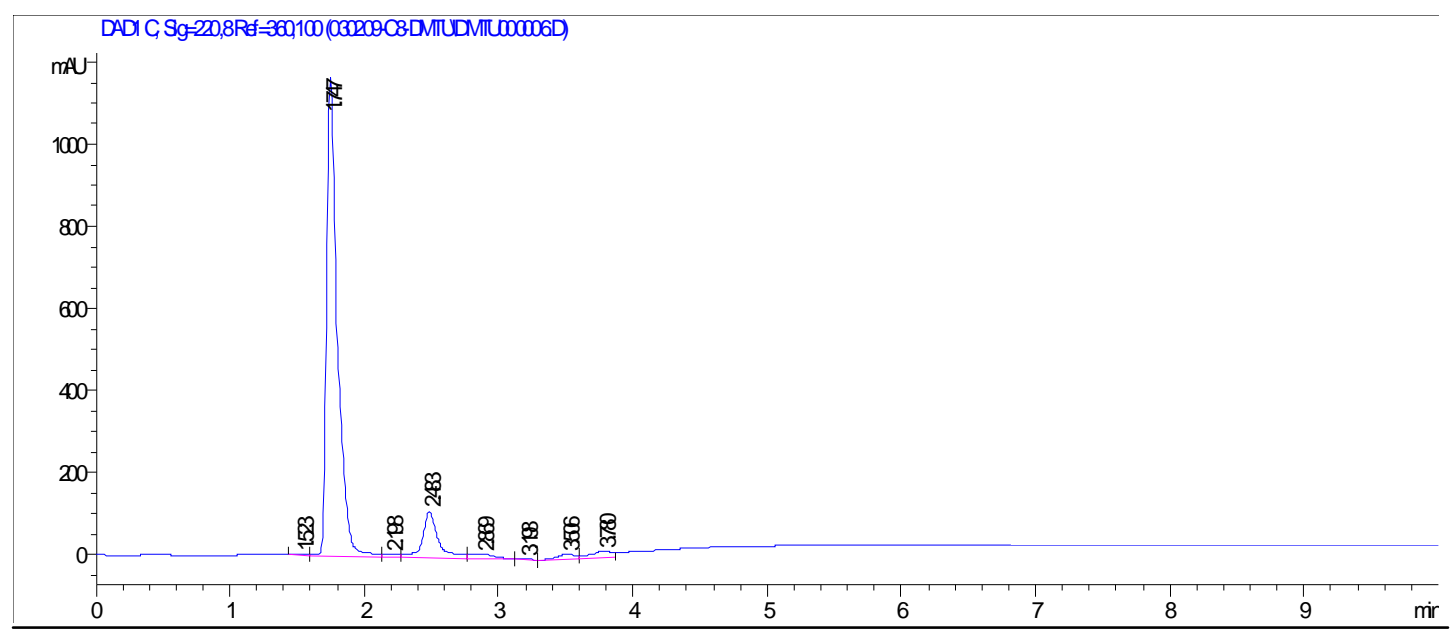

Figure 57. ANP isocratic retention of raloxifene on silica hydride-based C8 column. The isocratic composition used is 95:5 acetonitrile/water + 0.1\% TFA.

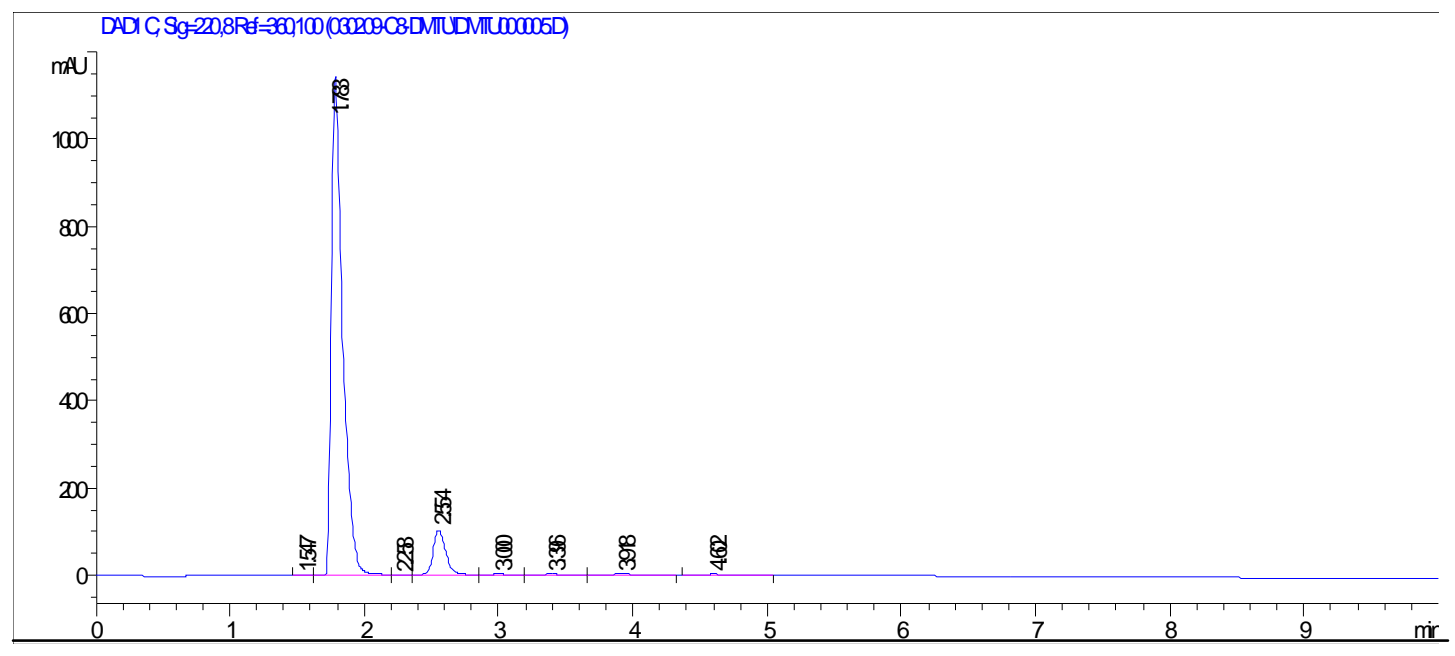

Figure 58. ANP isocratic retention of raloxifene on silica hydride-based C8 column. The isocratic composition used is 80:20 acetonitrile/water $+0.1 \%$ TFA. 


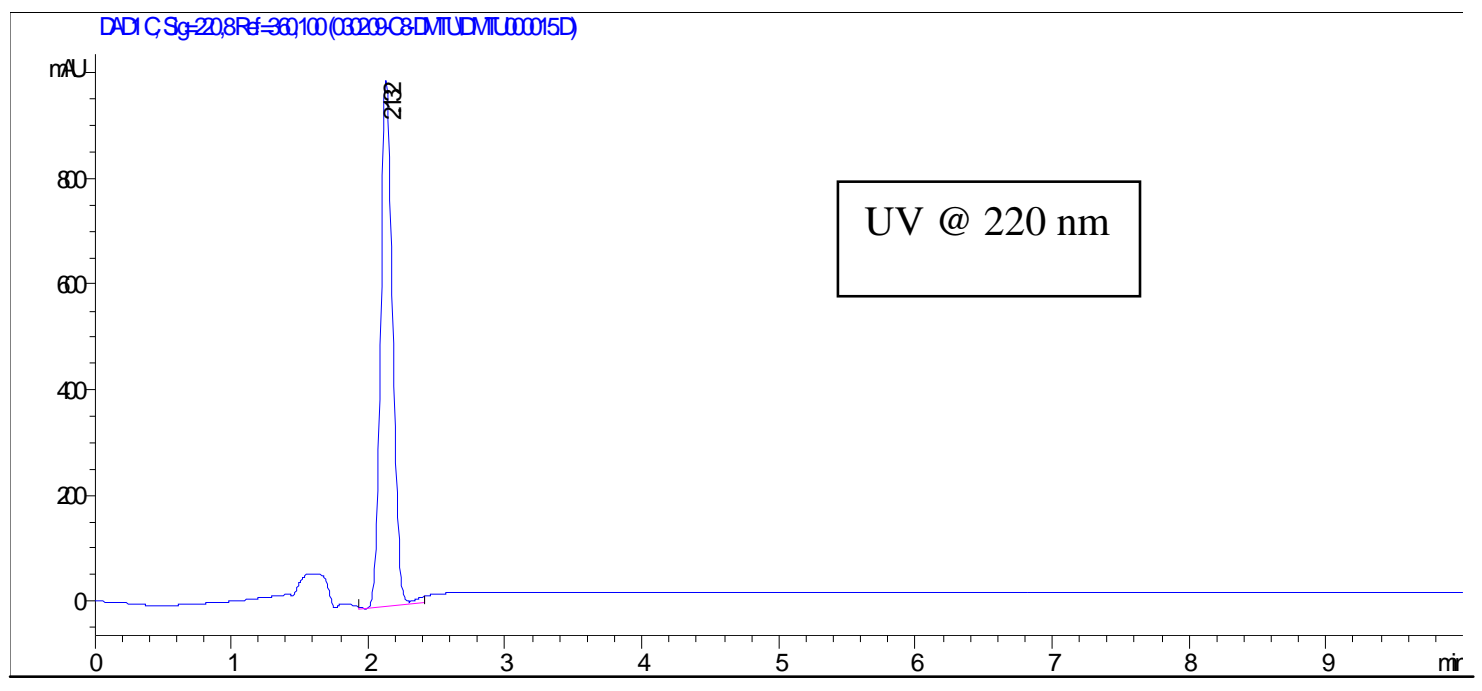

Figure 59. ANP isocratic retention of lipoic acid on silica hydride-based C8 column. The isocratic composition used is 80:20 acetonitrile/water + 0.1\% TFA.

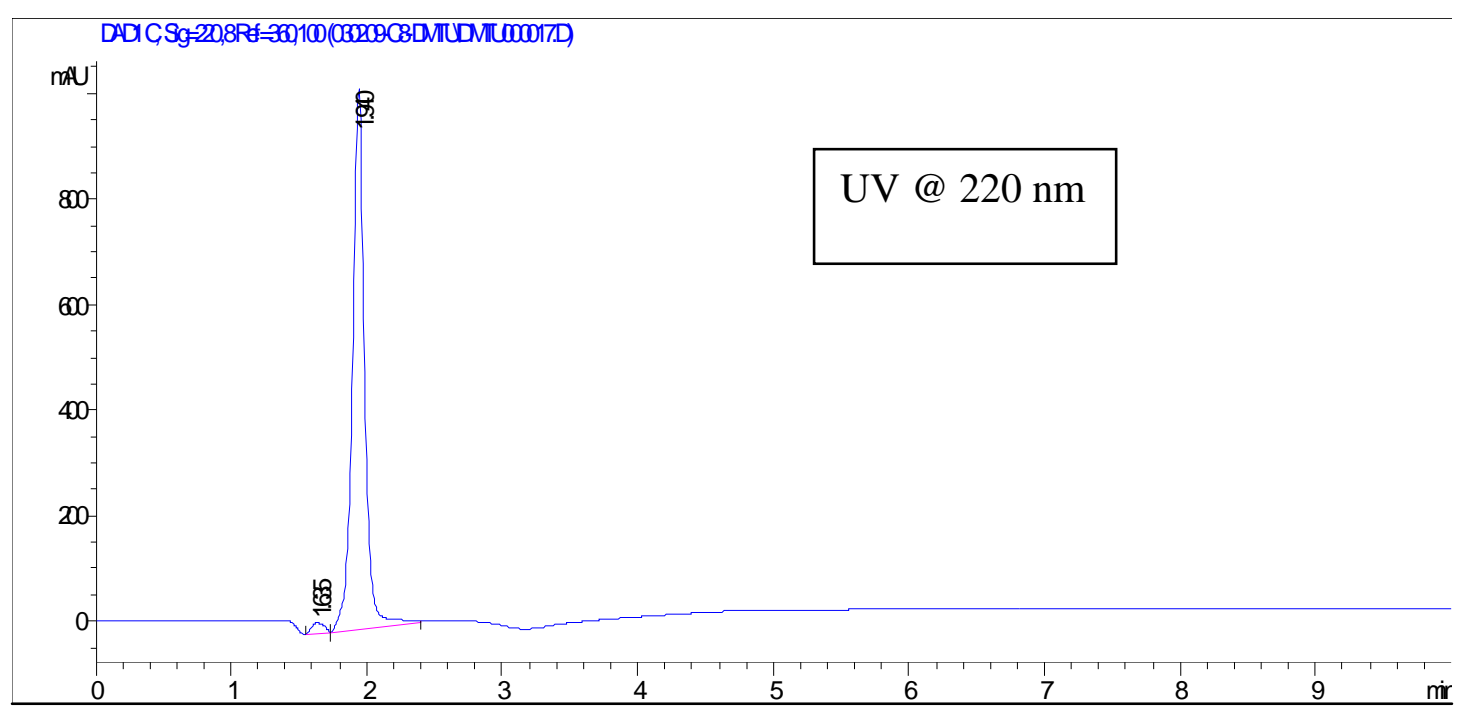

Figure 60. ANP isocratic retention of lipoic acid on silica hydride-based C8 column. The isocratic composition used is 95:5 acetonitrile/water + 0.1\% TFA. 
Raloxifene being nonpolar eluted early in the aqueous normal phase mode. The aqueous normal phase behavior data obtained for the $\mathrm{C} 8$ column confirms that the stationary phase was not suitable for aqueous normal phase separation of these compounds.

\section{b. Reversed-Phase Behavior}

Raloxifene, triphenylphosphine, lisinopril, and niacinic acid were analyzed under reversed-phase gradient conditions using silica hydride based C8 column as shown in Figures 61 to 66 . The concentration of the sample was $0.5 \mathrm{mg} / \mathrm{mL}$ and the detector used was UV.

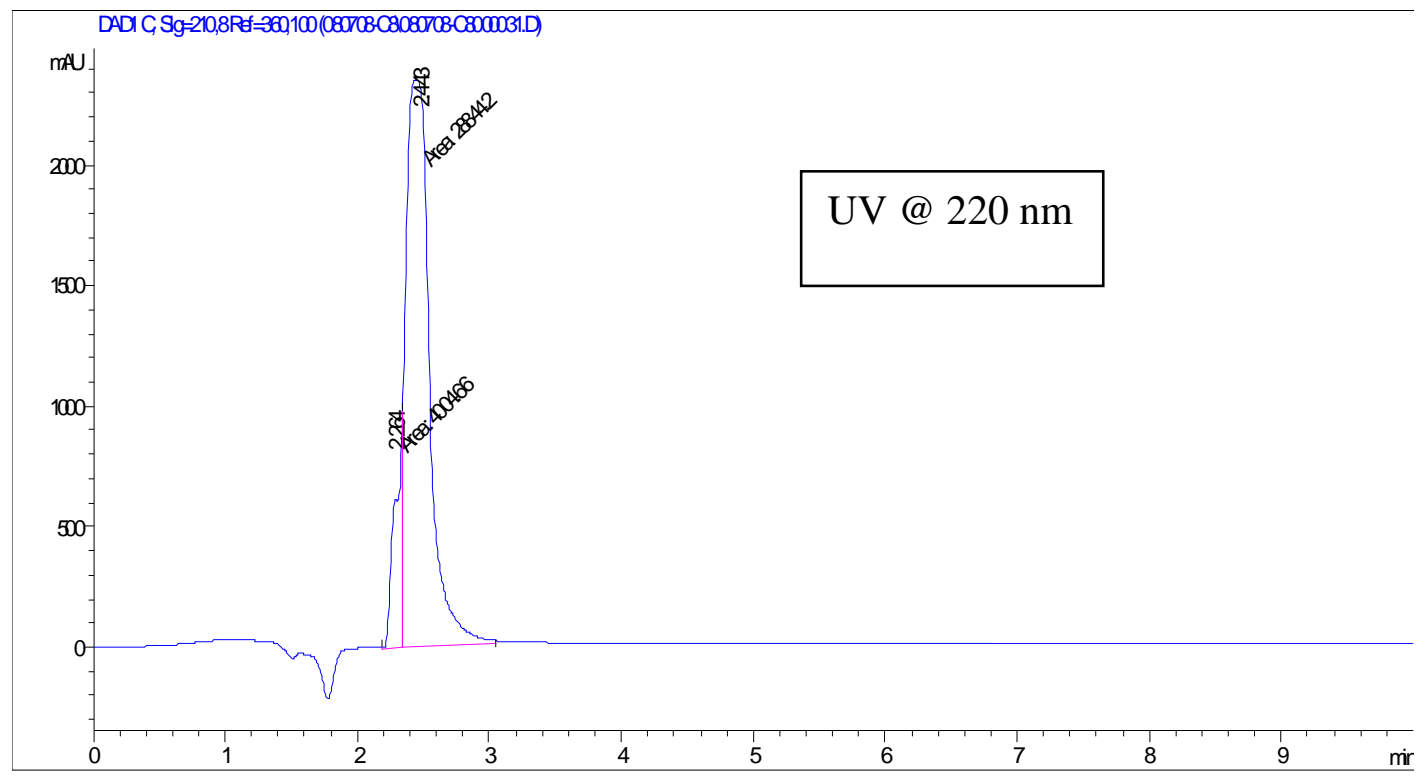

Figure 61. RP isocratic retention of niacinic acid on silica hydride-based C8 column. The isocratic composition used is $30: 70$ acetonitrile/water $+0.1 \%$ TFA. 


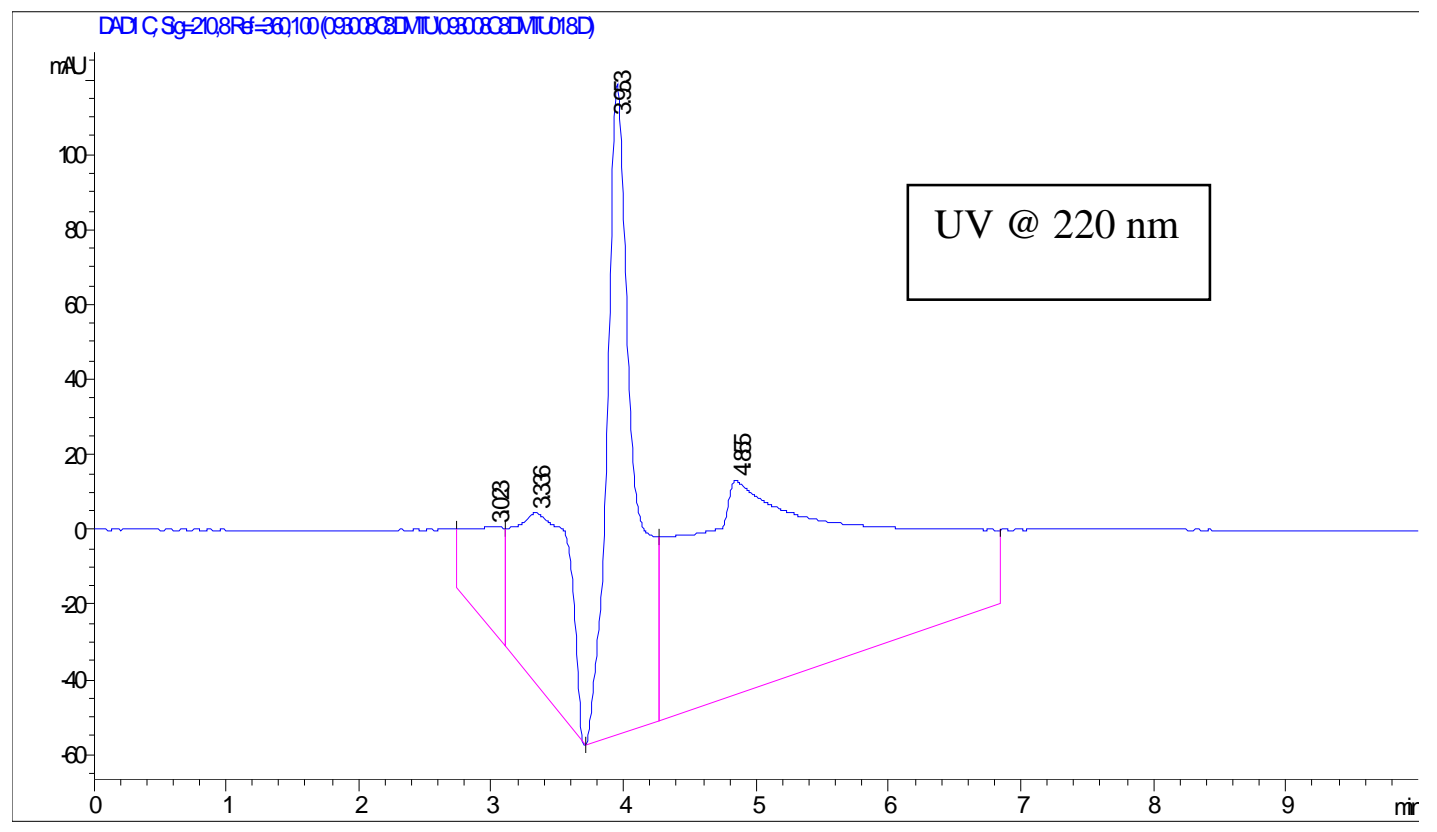

Figure 62. RP isocratic retention of lisinopril on silica hydride-based C8 column. The isocratic composition used is 45:55 acetonitrile/water + 0.1\% TFA. 


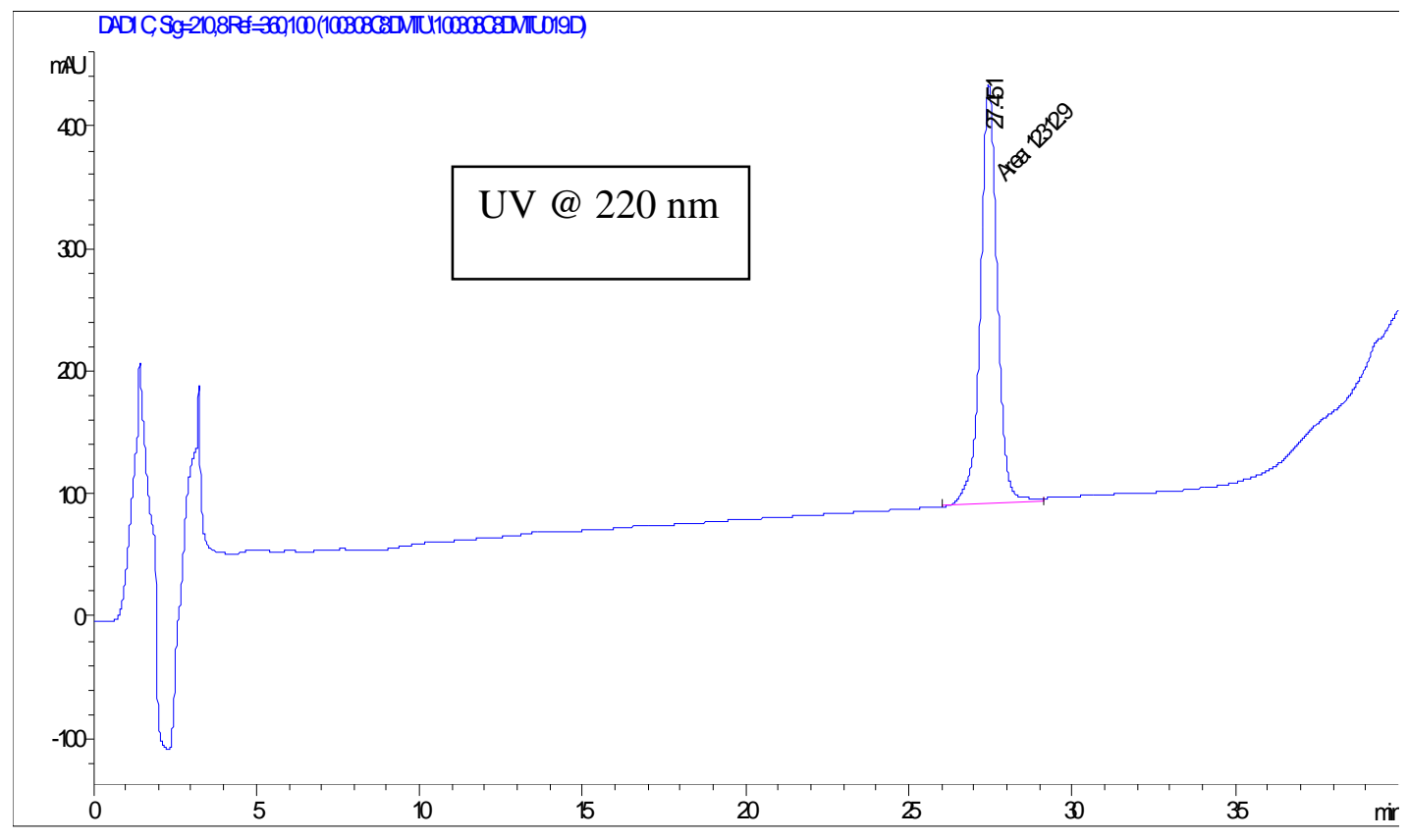

Figure 63. RP gradient retention of triphenylphosphine on the silica hydride-based C8 column. It elutes at $\mathbf{2 7 . 5}$ min with Gradient $\mathrm{C}$ as shown in Table 8. 


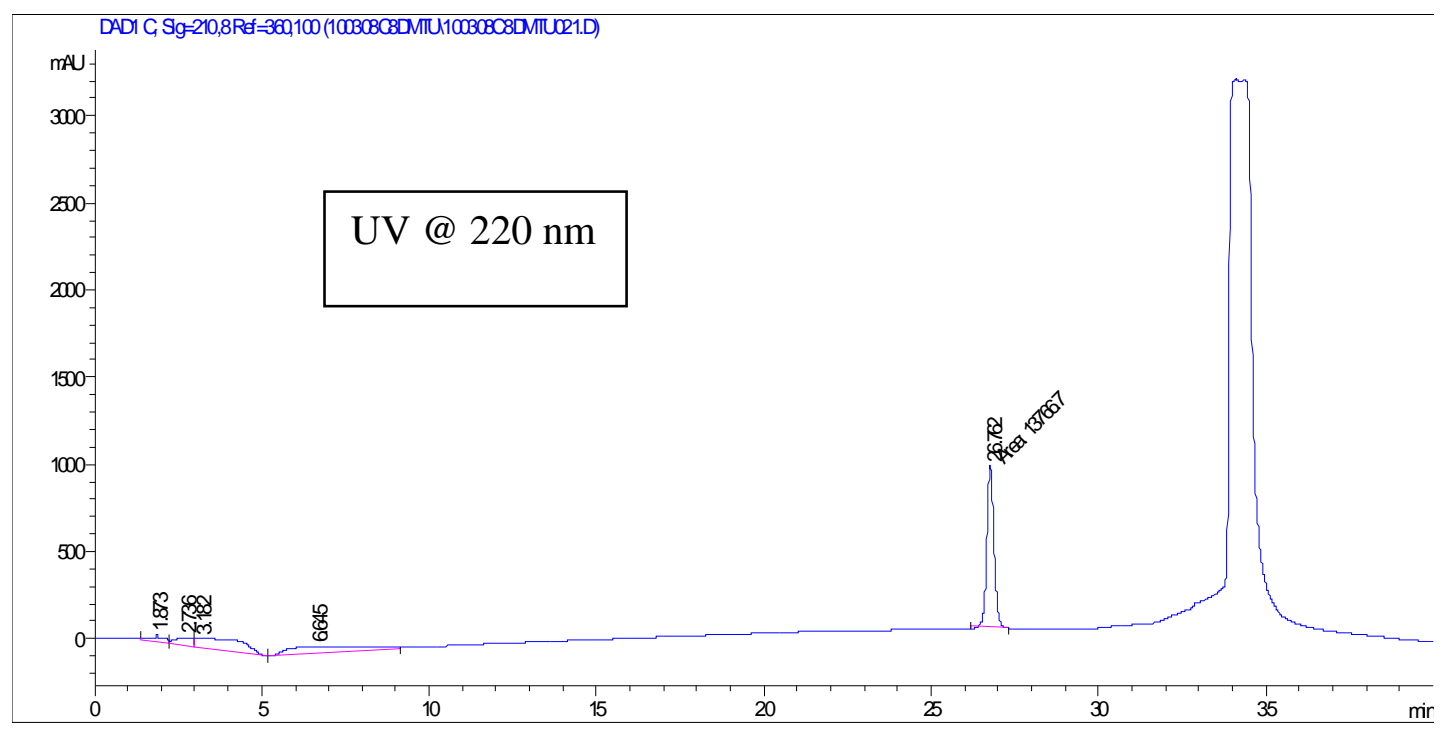

Figure 64. RP gradient retention of triphenylphosphine on silica hydride-based C8 column. It elutes at 26.7 min with Gradient B as shown in Table 7. 


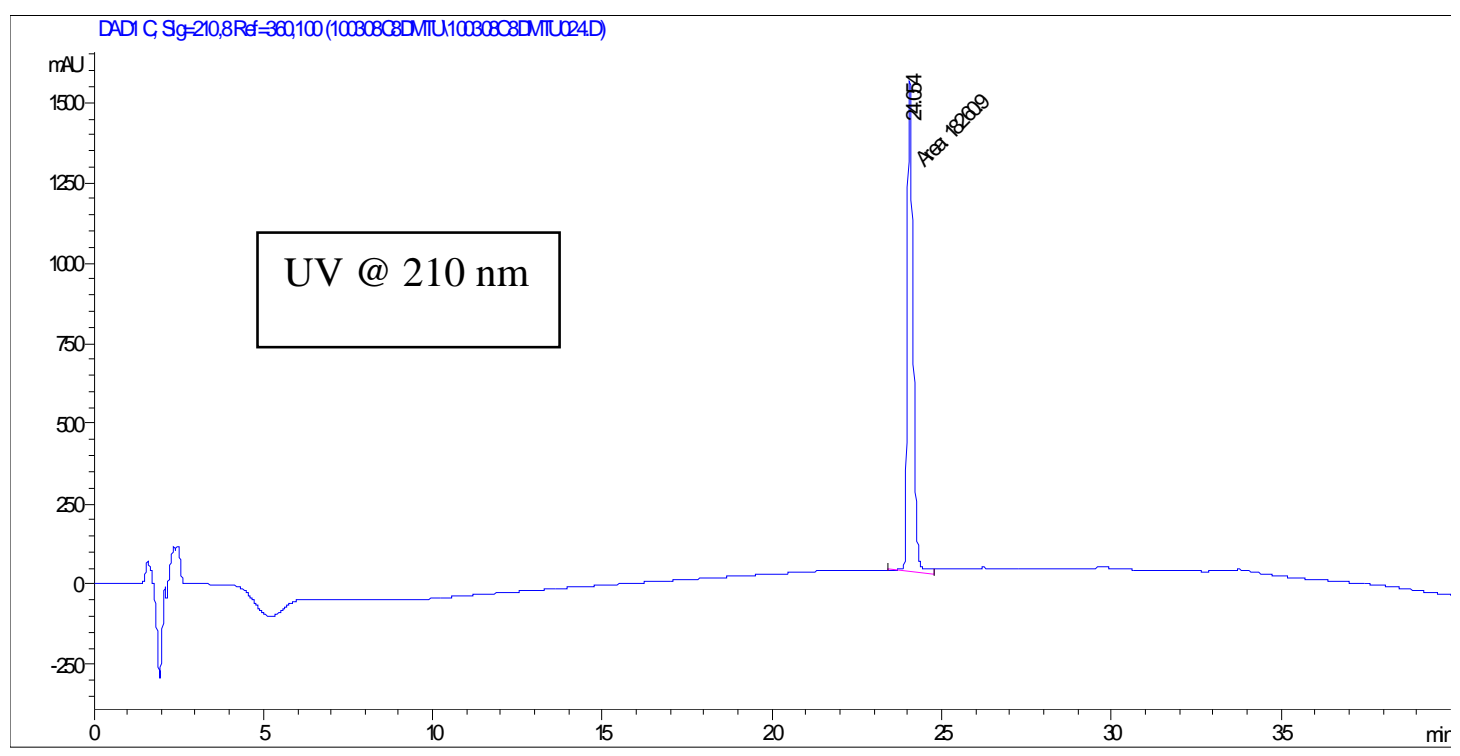

Figure 65. RP gradient retention of raloxifene on the silica hydride-based C8 column. It elutes at $\mathbf{2 4 . 0 6}$ min with Gradient B as shown in Table 7. 


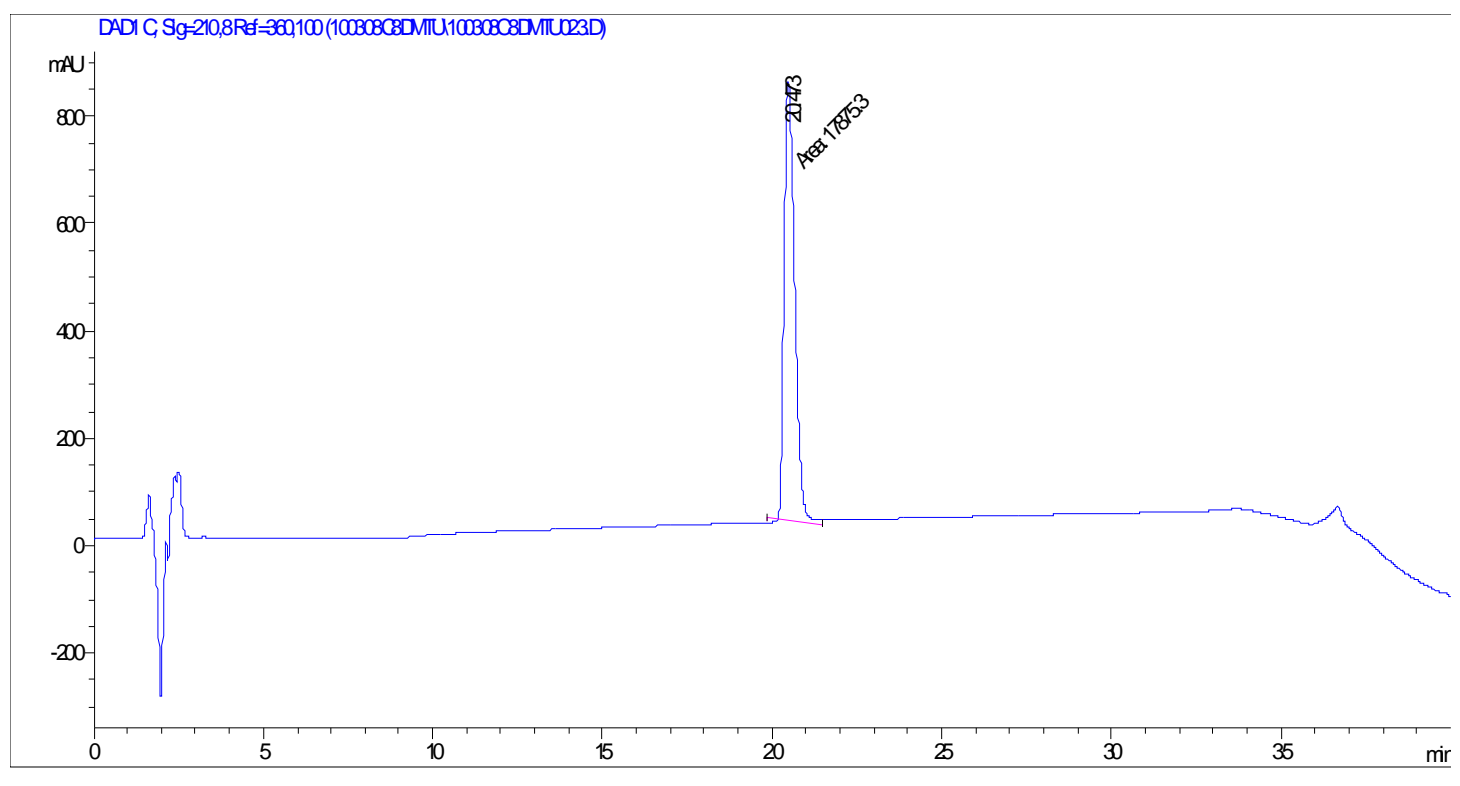

Figure 66. RP gradient retention of raloxifene on silica hydride-based C8 column. It elutes at 20.07 min with Gradient D as shown in Table 7.

Raloxifene and triphenylphosphine being very nonpolar were retained very well on the $\mathrm{C} 8$ column. The retention time varied from gradient to gradient depending on the increment of the percent acetonitrile composition in the mobile phase.

Triphenylphosphine being very nonpolar does not dissolve in any solvent except

cyclohexane. The peak eluting at $35 \mathrm{~min}$ in Figure 64 was due to cyclohexane.

Gradient B was used as shown in Table 7. Gradient C shown in Table 8 was used for the elution of triphenylphosphine as shown in Figure 63. Lisinopril and niacinic acid were measured using reversed-phase isocratic conditions as shown in Figures 61 and 62. The lisinopril chromatogram has a number of negative peaks that could be from the mobile phase. If the mobile phase has greater absorbance than the compound being analyzed, 
then it was possible to observe negative peaks. These negative peaks can be eliminated by choosing a different mobile phase or by optimizing the gradient conditions. Therefore more work needs to be done in order to improve the appearance of the chromatogram.

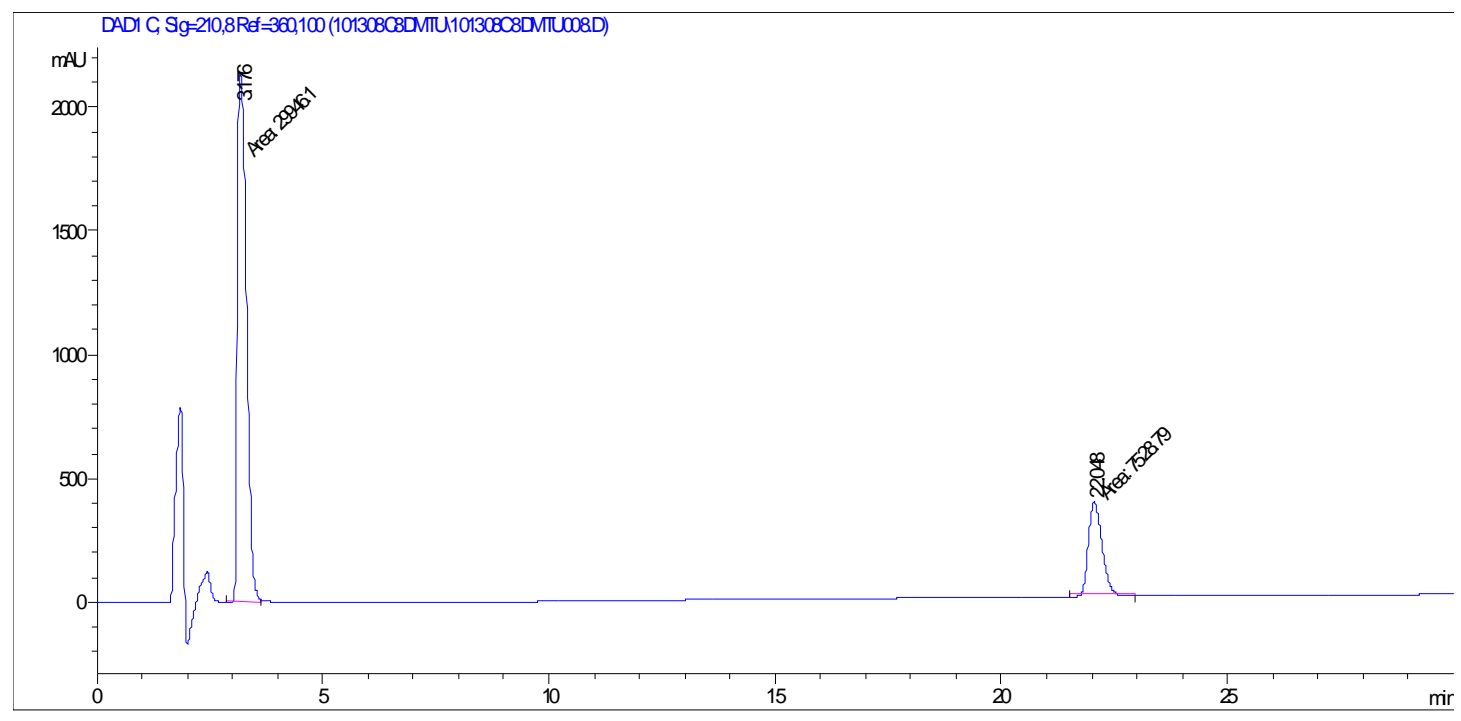

Figure 67. RP gradient separation of lisinopril and raloxifene on silica hydride based C8 column. Lisinopril elutes at $3.18 \mathrm{~min}$ and raloxifene elutes at $22.05 \mathrm{~min}$. Gradient $\mathrm{C}$ used for the separation is shown in Table 8.

A mixture of lisinopril and raloxifene was separated using Gradient $\mathrm{C}$ described in Table 8 under reversed-phase conditions as shown in Figure 67. The mobile phase flow rate was $0.5 \mathrm{~mL} / \mathrm{min}$ and $\mathrm{UV}$ detection at $220 \mathrm{~nm}$ was used. Lisinopril being less nonpolar compared to raloxifene eluted earlier in the gradient at $3.2 \mathrm{~min}$, and raloxifene being more nonpolar eluted at $22.05 \mathrm{~min}$. The retention time for each of the compounds 
was confirmed by running the single analyte using the same gradient, mobile phase, and instrument.

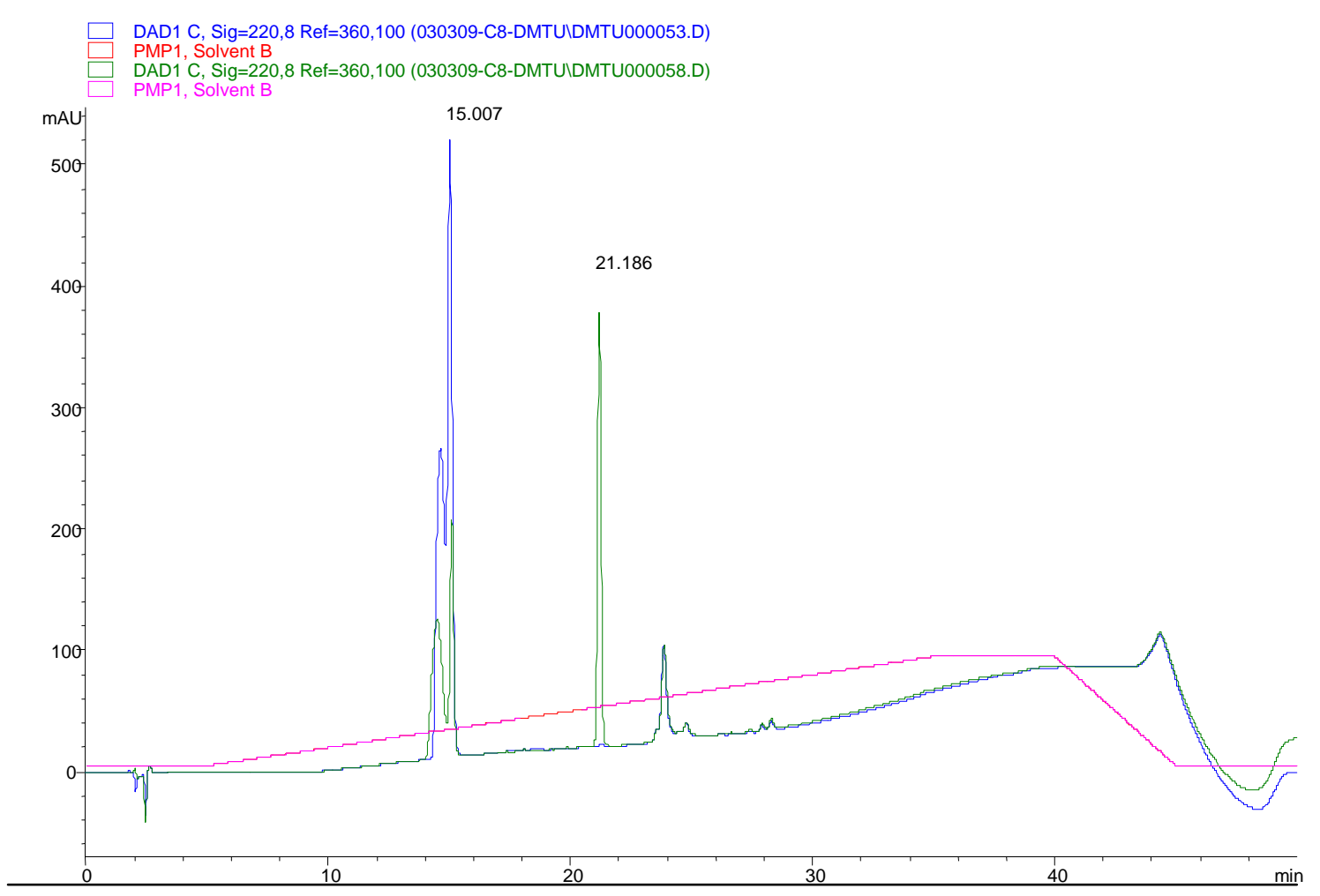

Figure 68. RP gradient separation of lisinopril and lipoic acid on silica hydride based C8 column. Lisinopril elutes at 15 min and lipoic acid elutes at $21.2 \mathrm{~min}$. Gradient B used for the separation is shown in Table 8.

The overlay of reversed-phase chromatograms of lisinopril and the mixture of lisinopril and lipoic acid was as shown in Figure 68. Lisinopril being less nonpolar compared to lipoic acid eluted earlier at $15 \mathrm{~min}$, while lipoic acid eluted at $21.2 \mathrm{~min}$. The 
lisinopril retention time was confirmed by injecting it as a single compound and overlaying the chromatogram with a mixture of two compounds. Gradient B was used for the separation and detection was done by UV.

\section{Comparison of Endcapped Si-H Column and C8 Column Separation Under RP Conditions}

The overlay of the chromatograms of raloxifene on the endcapped silica hydride column and the C8 column are shown in Figures 69 and 70. 


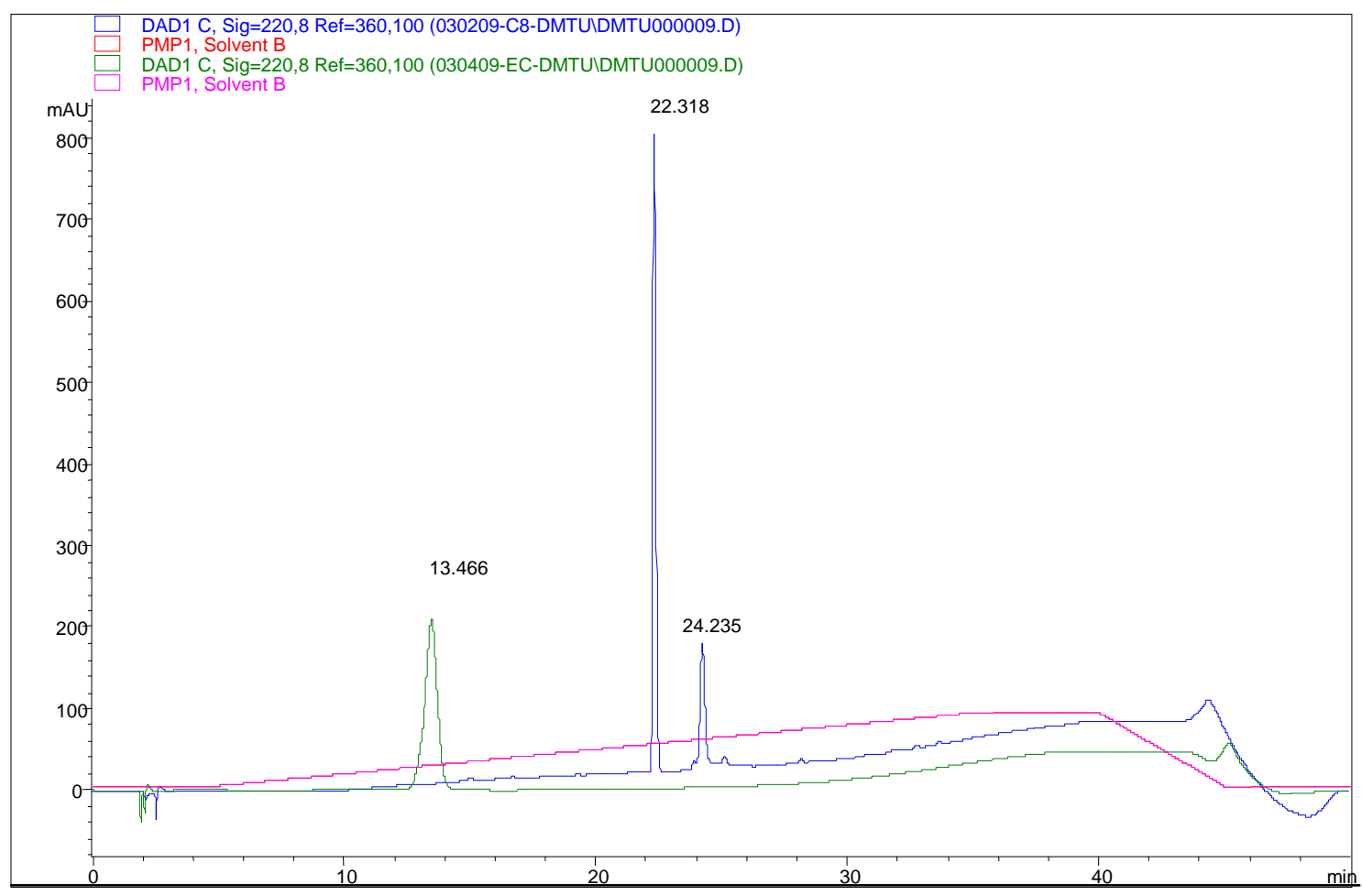

Figure 69. The overlay of RP chromatograms of raloxifene for endcapped Si-H and C8 column. The peak at $13.5 \mathrm{~min}$ is on the endcapped Si-H column and the peak at 22.32 min is on the C8 stationary phase. Gradient B used is shown in Table 7. 


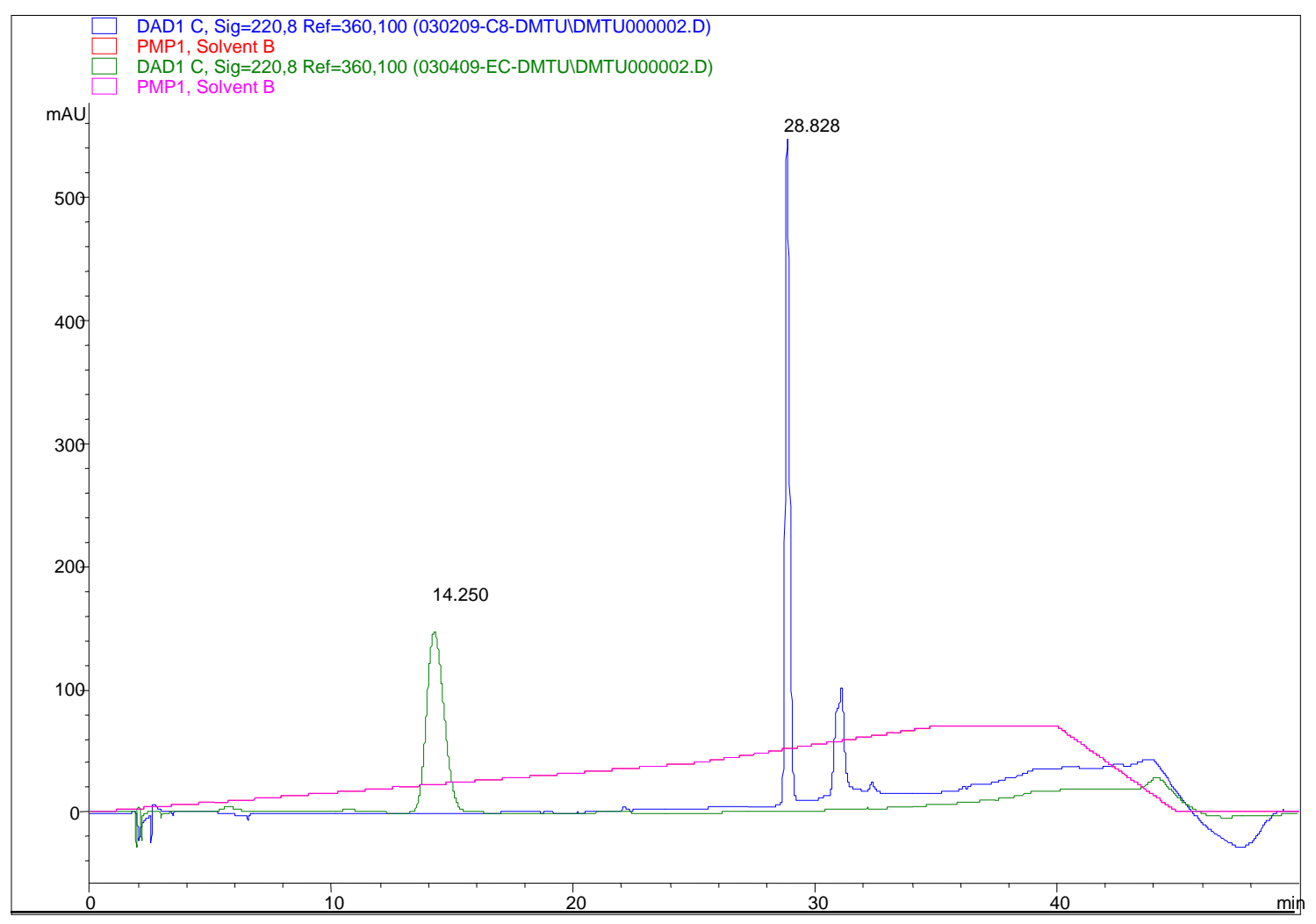

Figure 70. The overlay of RP chromatograms of raloxifene for endcapped Si-H and C8 column. The peak at $\mathbf{1 4 . 3} \mathbf{m i n}$ is on the endcapped Si-H column and the peak at 29 min is on the C8 stationary phase. Gradient A used is shown in Table 7.

Raloxifene being nonpolar in nature, so as expected it was retained longer on the C8 column than on the endcapped silica hydride column. Endcapping has definitely increased the hydrophobicity of the silica hydride, because it was able to reasonably retain the nonpolar compound, but to a lesser extent than on the C8 Si-H column. Raloxifene eluted at 13.5 min for the endcapped silica hydride column, and at 22.32 min for the C8 column as shown in Figure 69. The gradient used for the analysis was B as 
described in Table 7. Using gradient A as shown in Table 7, raloxifene was retained longer eluting at 14.3 min for the endcapped $\mathrm{Si}-\mathrm{H}$ column, and at $29 \mathrm{~min}$ for the $\mathrm{C} 8$ column as shown in Figure 70.

\section{Peak Symmetry Factor}

Chromatographic characterization also included an evaluation of the peak symmetry on the endcapped silica hydride column. The presence of residual silanol groups after silanization or after endcapping the $\mathrm{Si}-\mathrm{H}$ stationary phase, sometimes leads to peak tailing effects. The evaluation of the endcapping process, in regard to an increase in the efficiency of the silica hydride column separation, was determined by calculating the peak asymmetry factors of the endcapped silica hydride column. The samples when injected on the column using the HPLC system undergo a normal dispersion of the component molecules; this was represented by a bell-shaped Gaussian peak. In an ideal chromatographic elution the peak shape should be symmetric, but it is not possible to get symmetric peak shapes, because of tailing caused by unreacted silanol groups. The unwanted interaction of compounds with the residual silanol groups, causes the compounds to be retained in the column for longer periods of time, which causes peak tailing [12]. The peak asymmetry factor is defined as

$$
\mathrm{As}=\mathrm{b} / \mathrm{a}
$$

Where As is the asymmetry factor with the values a and b determined at $10 \%$ peak height. If the peak is symmetrical then $\mathrm{a}=\mathrm{b}$ and the asymmetry factor is one or close 
to one. As the asymmetry factor approaches one, then the peak symmetry is better. The determination of peak symmetry is shown in Figure 71 [34].

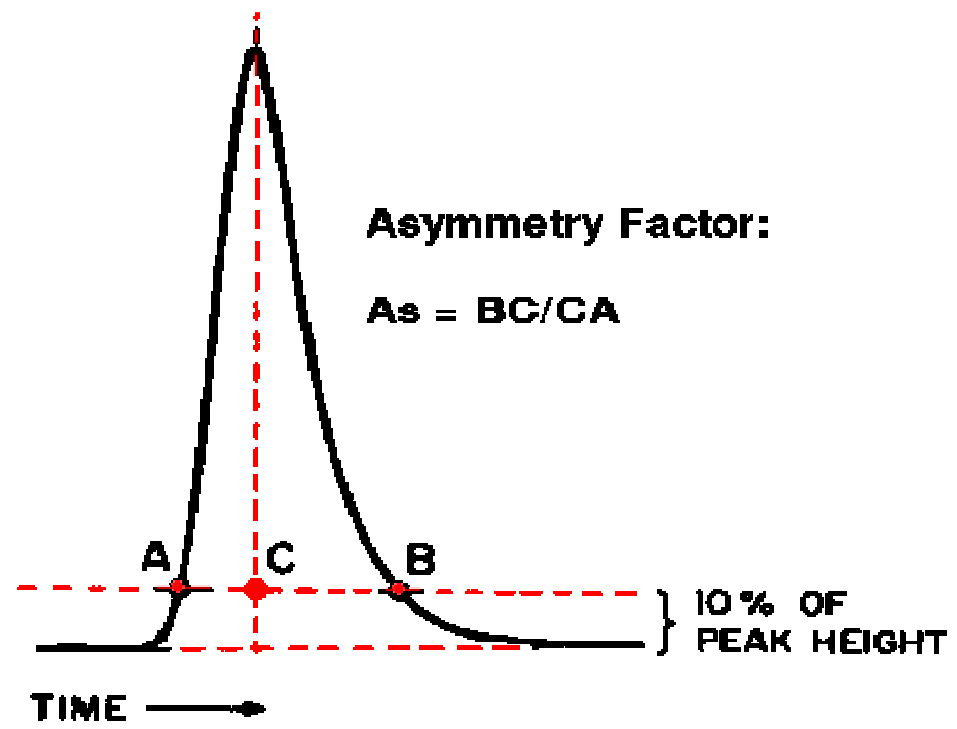

Figure 71. Determination of peak symmetry factor.

Figure 72 shows the plot of peak symmetry factor vs. \% acetonitrile for niacinic acid, citric acid, melamine, and phenylalanine on the endcapped silica hydride column using the ELSD detector. Figure 73 shows the plot of peak symmetry factor vs. \% acetonitrile for niacinic acid and phenylalanine for the endcapped silica hydride column using the UV detector. As shown in the figures, the peak symmetry factor is close to one for most percentages of acetonitrile. This indicates that the column efficiency was indeed greatly enhanced after the endcapping of the silica hydride stationary phase with trimethylchlorosilane and hexamethyldisilazane. As shown in Figure 72, melamine has a symmetry factor close to 5 for a $70 \%$ acetronitrile/30\% water mobile-phase composition. Melamine being very basic in nature, could be retained well under reversed-phase 
conditions as well as in the ANP mode. For phenylalanine as shown in Figure 73, the symmetry factor was close to 3 when the composition of the acetonitrile used was $95 \%$, but other concentrations it was close to one. It appeared to be that to retain phenylalanine and to get symmetry peak $10 \%$ water was required in the mobile phase.

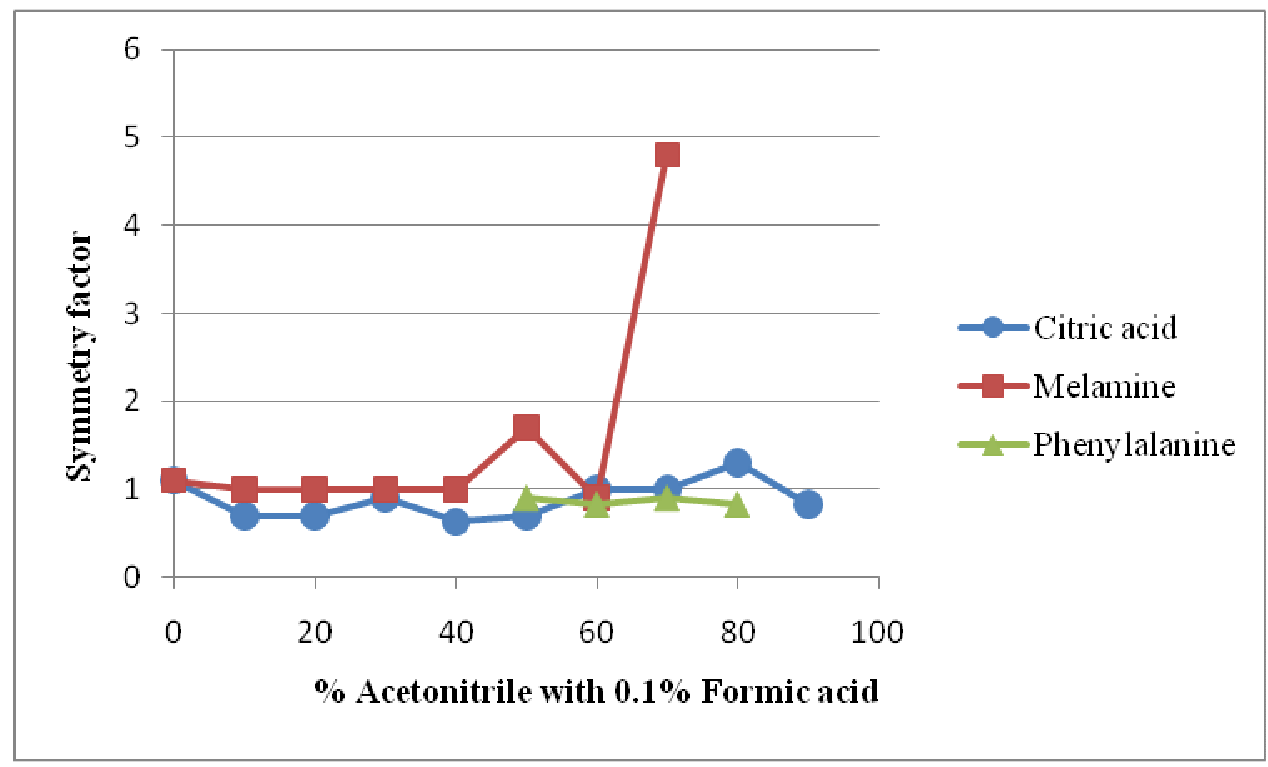

Figure 72. Chromatographic determination of asymmetry factors of compounds melamine, citric acid, and phenylalanine on endcapped silica hydride column using ELSD detection under isocratic conditions. 


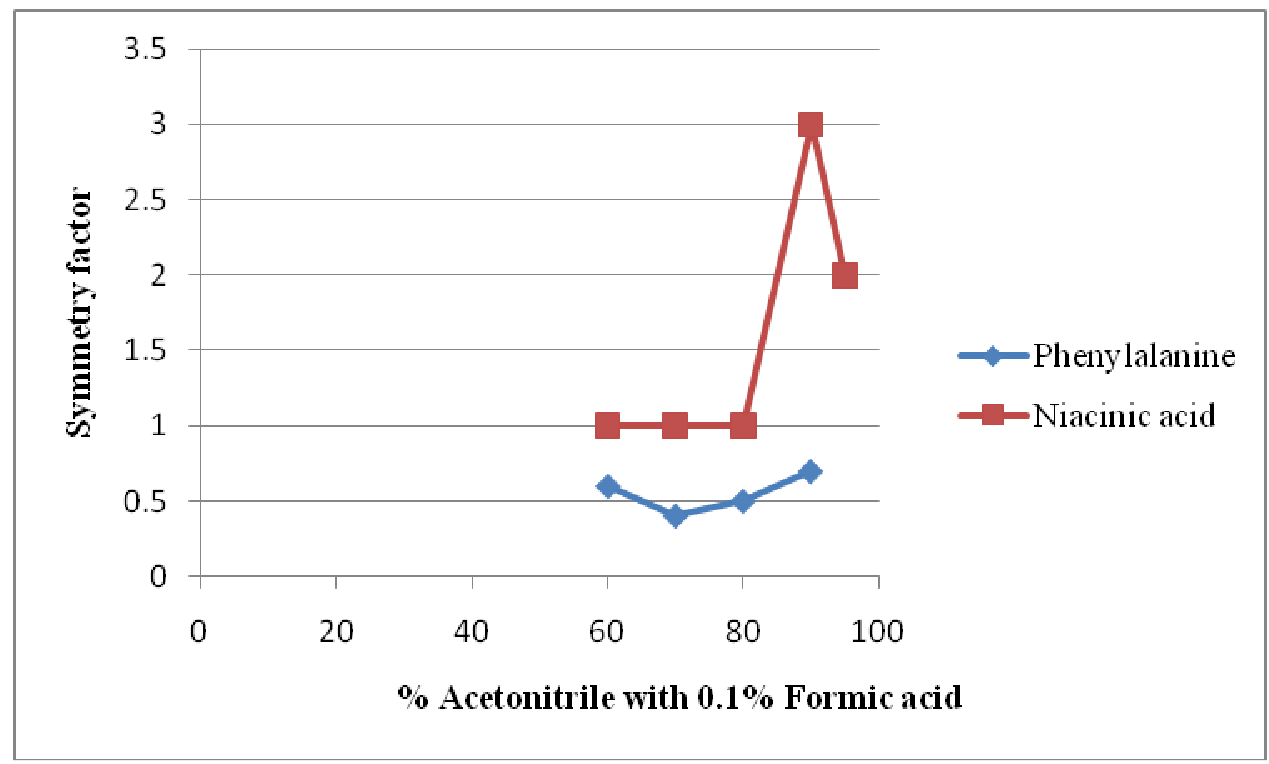

Figure 73. Chromatographic determination of asymmetry factors of the compounds niacinic acid and phenylalanine on endcapped silica hydride column using UV detection under isocratic conditions. 


\section{CONCLUSIONS}

For this research a silica hydride stationary phase was synthesized and endcapped with TMCS and HMDS. Spectroscopic characterization by DRIFT confirmed the success of the endcapping process. A Neue test evaluated the level of hydrophobicity to retain nonpolar compounds. The silanophilicity of the endcapped Si-H material confirmed the level of polar/adsorptive interactions with the surface. The hydrophobicity results of endcapped $\mathrm{Si}-\mathrm{H}$ column were comparable to the $\mathrm{C} 8 \mathrm{Si}-\mathrm{H}$ column.

The endcapped Si-H stationary phase showed good aqueous normal phase capabilities as well as some reversed-phase behavior. The results obtained on the endcapped column for the separation of nonpolar compounds were comparable with those from the $\mathrm{C} 8 \mathrm{Si}-\mathrm{H}$ column. The endcapped column showed a unique capability to use a wide range of mobile phase compositions starting from $100 \%$ aqueous to $100 \%$ nonpolar organic solvents. The endcapped column has the capability to rapidly change from reversed-phase to aqueous normal phase. Therefore, it was possible to operate both separation modes simultaneously, which helped in separating both hydrophobic and hydrophilic compounds. The peak symmetry factors calculated for some of the compounds confirmed that the column efficiency was greatly enhanced after the endcapping of the silica hydride stationary phase. Based on the results obtained from this research, it can be concluded that endcapping Si-H material with TMCS and HMDS was successful and showed comparable hydrophobicity with C8. 


\section{REFERENCES}

1. Skoog, A. D.; Holler, F. J.; Nieman, T. A. Principles of Instrumental Analysis, $5^{\text {th }}$ Ed. Saunders College Publishing, Orlando, Florida, 1998, Chapters 26, 28.

2. Pesek, J. J.; Matyska, M. T. Our Favorite Materials: Silica Hydride Stationary Phases. J. Sep. Sci. 2009, 32, 3999-4011.

3. Pesek, J. J.; Tang, H. V. The Modification of Alumina, Zirconia, Thoria and Titania for Potential Use as HPLC Stationary Phases through a Silanization/Hydrosilation Reaction Scheme. Chromatographia. 1994, 39, 11-12.

4. Snyder, L. R.; Kirkland, J. J.; Glajch, J. L. Practical HPLC Method Development. Wiley-Interscience, New York, 1997, 178-270.

5. Cunico, R. L.; Gooding, K. M.; Wehr, T. Basic HPLC and CE of Biomolecules. Bay Bioanalytical Laboratory, CA, 1998.

6. Dorsey, J. G.; Cooper, W. T. Retention Mechanisms of Bonded-Phase Liquid Chromatography. Anal. Chem. 1994, 66, 857A-867A.

7. Karen, M. G.; Fred, E. R.; Eds. HPLC of Biological Macromolecules. Marcel Dekker, Inc. New York, 1990, Chapters 6-11.

8. Pesek, J. J.; Sandoval, J. E.; Matyska, M. T. Synthesis, Characterization and Applications of Hydride-Based Surface Materials for HPLC, HPCE and Electrochromatography. J. Liq. Chrom. \& Rel. Technol. 1996, 19, 2843-2865. 
9. Sandoval, J. E.; Pesek, J. J. Hydrolytically Stable Bonded Chromatographic Phases Prepared through Hydrosilation of Olefins on a Hydride-Modified Silica Intermediate. Anal. Chem. 1991, 2634-2641.

10. Kirkland, J. J.; Glajch, J. L.; Farlee, R. D. Synthesis and Characterization of Highly Stable Bonded Phases for High Performance Liquid Chromatography Column Packings. Anal. Chem. 1986, 61, 2-11.

11. Chu, C. H.; Jonsson, E.; Auvinen, M.; Pesek, J. J.; Sandoval, E. J. A new Approach for the Preparation of a Hydride-Modified Substrate Used as an Intermediate in the Synthesis of Surface-Bonded Materials. Anal. Chem. 1993, 65, 808-816.

12. Pesek, J. J.; Matyska, M. T. Williamsen, J. E.; Evanchic, M.; Hazari, V.; Konjuh, K.; Takhar, S.; Rosalia T. Synthesis and Characterization of Alkyl Bonded Phases from a Silica Hydride via Hydrosilation with Free Radical Initiation. $J$ Chromatogr. A. 1997, 786, 219-228.

13. Akapo, S. O.; Dimandja, J. D. Improvement of Gas-Chromatographic Performance of Cyclic Siloxane Bonded Phases by Incorporation of n-alkyl Groups. Anal. Chem. 1996, 68, 1954-1959.

14. Sander, L. C.; Wise, S. A. Recent Advances in Bonded Phases for LiquidChromatography. CRC Critical Reviews in Analytical Chemistry. 1987, 18, 319. 
15. Pesek, J. J.; Matyska, M. T. Raymond J. Y. Synthesis and Characterization of Endcapped C18 Stationary Phases using a Silica Hydride Intermediate. $J$. Chromatogr. 2002, 947, 195-203.

16. Uwe D. N.; Kim V. T.; Pamela C. I.; Bonnie A. A. Characterization of HPLC Packings. J. Sep. Sci. 2003, 26, 174-186.

17. Berendsen, G. E.; De Galan, L. Preparation and Chromatographic Properties of Some Chemically Bonded Phases for Reversed-Phase Liquid Chromatography. $J$. Liq. Chromatogr. 1978, 1, 561-568.

18. Radha, S. Synthesis and characterization of amino based columns for HPLC. M.S. Thesis, San Jose State University, 2004.

19. Yan, D.; Li, G.; Xiao, X. H.; Dong, X. P.; Li, Z. L. Direct Determination of Fourteen Underivatized Amino Acids from Whitmania Pigra by using Liquid Chromatography-Evaporative Light Scattering Detection. J Chromatogr. A. 2007, 1138, 301-304.

20. SOFTA CORPORATION, Model 400 ELSD Manual, version 1.2, August 2006. http://www.softacorporation.com (accessed Sep 20, 2010).

21. Acenaphthene. http://en.wikipedia.org/wiki/Acenaphthene (accessed Sep 20, 2010).

22. Naphthalene. http://en.wikipedia.org/wiki/Naphthalene (accessed Sep 20, 2010).

23. Uracil. http://en.wikipedia.org/wiki/Uracil (accessed Sep 20, 2010).

24. Amitriptyline. http://en.wikipedia.org/wiki/Amitriptyline (accessed Sep 20, 2010). 
25. Lisinopril. http://en.wikipedia.org/wiki/Lisinopril (accessed Sep 20, 2010).

26. Raloxifene. http://en.wikipedia.org/wiki/Raloxifene (accessed Sep 20, 2010).

27. Dimethylthiourea. http://www.chemexper.com/chemicals/supplier/cas/534-134.html (accessed Sep 21, 2010).

28. Lipoic acid. http://en.wikipedia.org/wiki/Lipoic_acid (accessed Sep 21, 2010).

29. Triphenylphosphine. http://en.wikipedia.org/wiki/Triphenylphosphine (accessed Sep 21, 2010).

30. Phenylalanine. http://en.wikipedia.org/wiki/Phenylalanine (accessed Sep 21, 2010).

31. Melamine. http://en.wikipedia.org/wiki/Melamine (accessed Sep 21, 2010).

32. Citric acid. http://en.wikipedia.org/wiki/Citric_acid (accessed Sep 21, 2010).

33. Niacin. http://en.wikipedia.org/wiki/Niacin (accessed Sep 21, 2010).

34. Asymetry factor. http://www.lcresources.com/resources/TSWiz/hs170.htm (accessed Sep 25, 2010) 